Aus der Klinik für Psychiatrie und Psychotherapie

(Prof. Dr. med. J. Wiltfang)

der Medizinischen Fakultät der Universität Göttingen

\title{
Sexuelle Gesundheit in der Altenpflege
}

\author{
INAUGURAL-DISSERTATION \\ zur Erlangung des Doktorgrades \\ der Medizinischen Fakultät der \\ Georg-August-Universität zu Göttingen
}

vorgelegt von

Juliana Therèse Warneck

aus

Berlin

Göttingen 2020 
Dekan:

\section{Betreuungsausschuss}

Betreuer:

Ko-Betreuer:

\section{Prüfungskommission}

Referent:

Ko-Referent:

Drittreferentin:

Promotor-Vertreterin:
Prof. Dr. med. W. Brück

Prof. Dr. med. J. Wiltfang

Prof. Dr. rer. pol. W. Himmel
Prof. Dr. J. Wiltfang

Prof. Dr. W. Himmel

Prof. Dr. C. von Arnim

Prof. Dr. M. Schön

Datum der mündlichen Prüfung: 28.07.2021 
Hiermit erkläre ich, die Dissertation mit dem Titel „Sexuelle Gesundheit in der Altenpflege“ eigenständig angefertigt und keine anderen als die von mir angegebenen Quellen und Hilfsmittel verwendet zu haben.

Göttingen, den 16.07.2020 


\section{Inhaltsverzeichnis}

Abbildungsverzeichnis ................................................................................ III

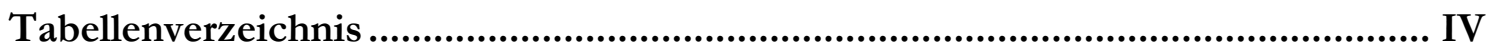

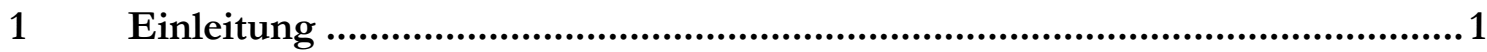

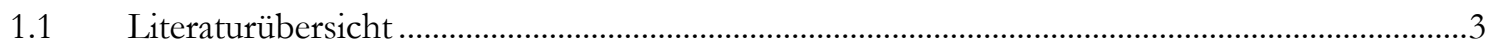

1.2 Ausbildung und Lernfelder in der Altenpflege .......................................................................6

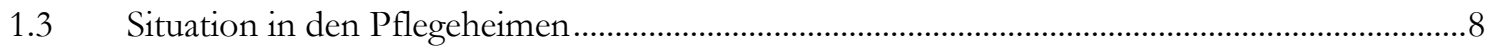

1.4 Pflegestatistik im Kontext des demographischen Wandels in Deutschland ..............................

$1.5 \quad$ Fragestellungen und Zielsetzung der Arbeit ...................................................................... 12

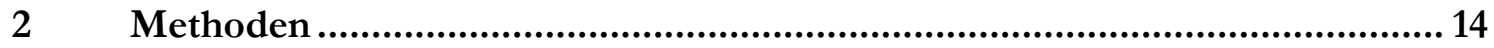

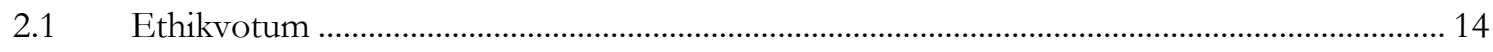

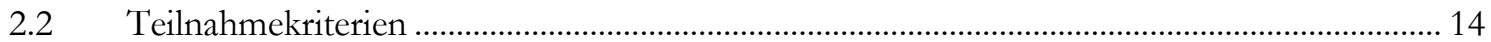

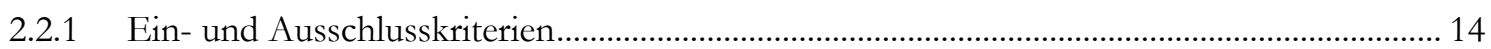

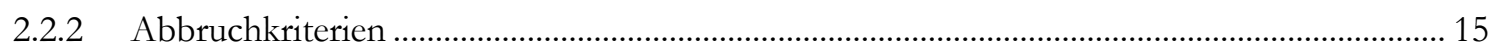

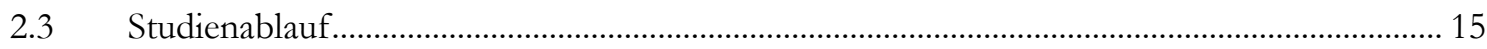

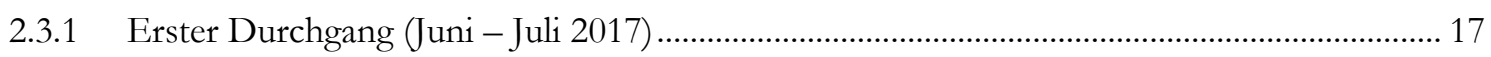

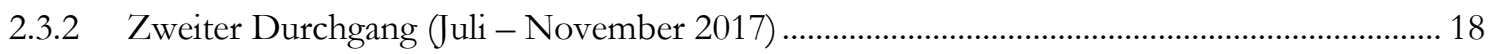

2.4 Fragebogen der Auszubildenden und Altenpflegenden ........................................................... 19

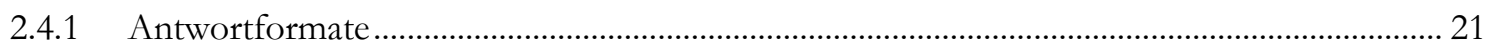

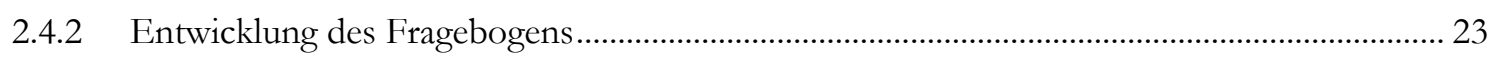

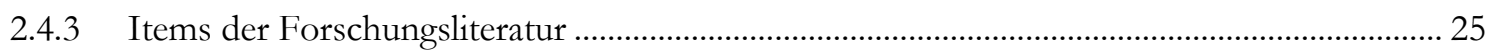

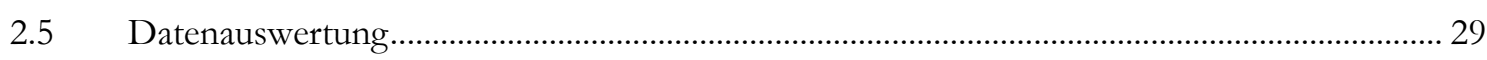

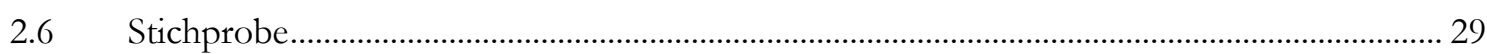

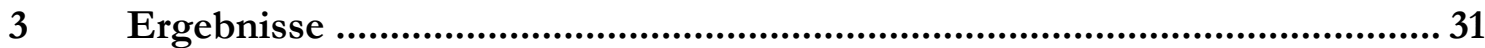

3.1 Q 1: Welchen Stellenwert hat das Thema Sexualität und sexuelle Gesundheit in der

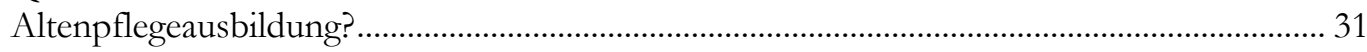

3.2 Q 2: Welchen Stellenwert hat das Thema Sexualität und sexuelle Gesundheit im

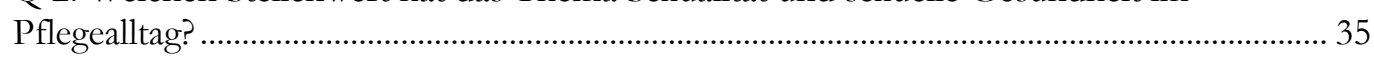

3.3 Q 3: Was ist der aktuelle Wissensstand in der Altenpflege zum Thema Sexualität und

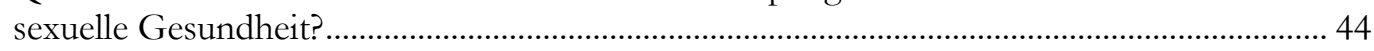

3.4 Q 4: Wie wird die eigene Sexualität, die Sexualität älterer Menschen und die Sexualität eigener Angehöriger wahrgenommen? ..................................................................................... 45

3.5 Q 5: Wie werden die Themen Sexualität im Elternhaus und Sexualaufklärung behandelt?

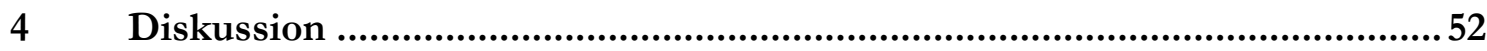

4.1 Stichprobe

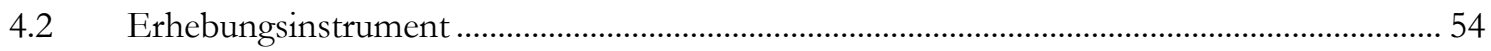

4.3 Q 1: Welchen Stellenwert hat das Thema Sexualität und sexuelle Gesundheit in der Altenpflegeausbildung? .......................................................................................................... 57 
4.4 Q 2: Welchen Stellenwert hat das Thema Sexualität und sexuelle Gesundheit im Pflegealltag?

4.5 Q 3: Was ist der aktuelle Wissensstand in der Altenpflege zum Thema Sexualität und sexuelle Gesundheit?

4.6 Q 4: Wie wird die eigene Sexualität, die Sexualität älterer Menschen und die Sexualität eigener Angehöriger wahrgenommen?

4.7 Q 5: Wie werden die Themen Sexualität im Elternhaus und Sexualaufklärung behandelt?

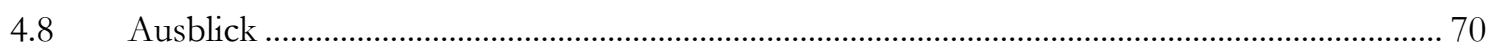

$5 \quad$ Zusammenfassung............................................................................... 72

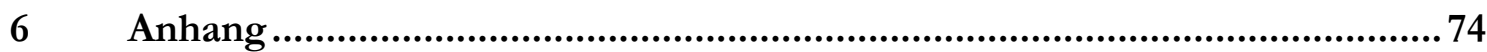

6.1 Fragebogen Auszubildende/Altenpflegende ................................................................. 74

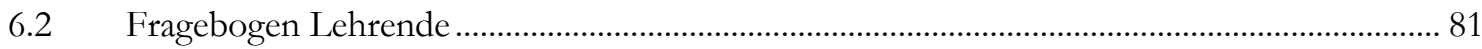

$7 \quad$ Literaturverzeichnis ..........................................................................90

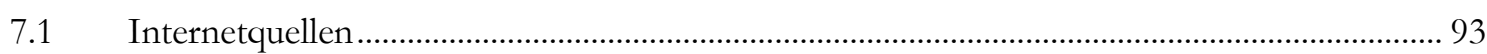




\section{Abbildungsverzeichnis}

Abbildung 1 Pflegebedürftige ab 65 Jahre nach Versorgungsart zum Jahresende 201310

Abbildung 2 Pflegebedürftige zum Jahresende 2015

Abbildung 3 Erster Durchgang (Juli 2017) 18

Abbildung 4 Zweiter Durchgang (November 2017) 19

Abbildung 5 Ich fühle mich gut vorbereitet auf den Umgang mit verbal und körperlich sexuell übergriffigem Verhalten von Heimbewohnern 31

Abbildung 6 Das Thema „Umgang mit Alterssexualität" wurde bei mir in der Ausbildung behandelt

Abbildung 7 Das Thema „Umgang mit Alterssexualität" als Ausbildungsfach halte ich für hilfreich für den Arbeitsalltag

Abbildung 8 Sexuelle Übergriffe von Heimbewohnenden auf Pflegende 38

Abbildung 9 Sexuelle Übergriffe von Pflegepersonal auf Heimbewohnende 38

Abbildung 10 Sexuelle Übergriffe unter Heimbewohnenden 38

Abbildung 11 Sexuelle Aktivität unter Heimbewohnenden $\quad 42$

Abbildung 12 Neubildung von Partnerschaften 42

Abbildung 13 Wieviel, meinen Sie, wissen Altenpflegende/Heimbewohnende im Durchschnitt über sexuell übertragbare Erkrankungen? 44

Abbildung 14 Ich kann meine Eltern/Großeltern als sexuell aktiv akzeptieren 46

Abbildung 15 Fragen zur eigenen Sexualität (1) 47

Abbildung 16 Fragen zur eigenen Sexualität (2) 48

Abbildung 17 Offenheit in der Familie 49

Abbildung 18 Wie häufig wurde in meiner Familie über Sexualität gesprochen? 49

Abbildung 19 Für wie aufgeklärt halte ich mich persönlich? 


\section{Tabellenverzeichnis}

Tabelle 1 Betreuungsschwerpunkte der Pflegeheime $\quad 8$

Tabelle 2 Arbeitsschwerpunkte der im Pflegeheim Beschäftigten 9

Tabelle 3 Anzahl der Pflegebedürftigen zum Jahresende 2013

Tabelle 4 Alter und Geschlecht der Teilnehmenden $\quad 30$

Tabelle 5 Vermittlung des Themas Alterssexualität: Gewünscht 33

Tabelle 6 Vermittlung des Themas Alterssexualität: Nicht gewünscht 33

Tabelle 7 Erweiterung des Themas Alterssexualität im Unterricht 34

Tabelle 8 Verringerung/Aussparung des Themas Alterssexualität im Unterricht 34

Tabelle 9 Am Arbeitsplatz habe ich eine offizielle Anlaufstelle, um über sexuelle
Vorkommnisse/Probleme in der Bewohnerversorgung zu reden

Tabelle 10 Paarbildung in meiner Einrichtung/meinem Wohnbereich 37

Tabelle 11 Häufigkeit: Auffinden sexueller Hilfsmittel 39

Tabelle 12 Reale und sinnvolle Bereitstellung sexueller Hilfsmittel in Einrichtungen $\quad 40$

Tabelle 13 Bereitstellung sexueller Hilfsmittel durch die Pflegeeinrichtung 41

Tabelle 14 Mit welchen der folgenden sexuell übertragbaren Erkrankungen hatten Sie in Ihrem Berufsalltag in der Altenpflege schon zu tun? 43

Tabelle 15 Gönnen Sie es alten Menschen, ihre Sexualität aktiv auszuleben? 45

Tabelle 16 Das aktive Ausleben von Sexualität im Alter ist etwas Natürliches 46

Tabelle 17 Von wem bin ich hauptsächlich aufgeklärt worden? $\quad 50$

Tabelle 18 Von wem bin ich hauptsächlich aufgeklärt worden? Aufteilung nach
Geschlechtszugehörigkeit

Tabelle 19 Über Folgendes bin ich von meinen Eltern aufgeklärt worden 51 


\section{$1 \quad$ Einleitung}

Die vorliegende Arbeit beschäftigt sich mit der Aktualität der Thematik „Sexuelle Gesundheit in der Altenpflege“. Die Erörterung des Studienthemas erfolgte anhand einer in zwei Durchgängen durchgeführten prospektiven Fragebogenstudie in und um Göttingen (Umkreis ca. $150 \mathrm{~km}$ ). Es wurden die Betrachtungsweisen von examinierten und auszubildenden Altenpflegenden an Altenpflegeheimen und -schulen zum Thema sexuelle Gesundheit und Alterssexualität angesichts ihrer Erfahrungen, unter besonderer Berücksichtigung der stationären Heimversorgung, erhoben. Hauptziel der Dissertation war es, mithilfe einer in der Arbeitsgruppe konzipierten Fragebogenstudie ein Meinungsbild zum Thema sexuelle Gesundheit in der Altenpflege zu erheben. Weiterhin galt es im Rahmen der Studie eine Bestandsaufnahme durchzuführen, um das Wissen zu Sexualität und sexueller Aufklärung sowie die subjektive Einstellung der Teilnehmenden zum Thema Sexualität zu ermitteln. Auch wurden die Einschätzungen von Lehrenden zum Thema Alterssexualität in der Altenpflegeausbildung betrachtet.

Den Schwerpunkt der vorliegenden Dissertation bildet das Thema sexuelle Gesundheit in der Altenpflege, weshalb zunächst eine eindeutige Definition gefordert ist. Die Weltgesundheitsorganisation (2011) definiert sexuelle Gesundheit als:

Einen Zustand des körperlichen, emotionalen, mentalen und sozialen Woblbefindens in Bezug auf die Sexualität und nicht nur das Feblen von Krankheit, Funktionsstörungen oder Gebrechen. Sie setz.t eine positive und respektvolle Haltung zu Sexualität und sexuellen Beziehungen voraus sowie die Möglichkeit, angenehme und sichere sexuelle Erfahrungen zu machen, und zwar frei von Zwang, Diskriminierung und Gewalt. Sexuelle Gesundheit lässt sich nur erlangen und erbalten, wenn die sexuellen Rechte aller Menschen geachtet, geschützt und erfüllt werden.

Sexuelle Gesundheit beschreibt daher nicht ausschließlich den Geschlechtsverkehr, sondern vielmehr die Möglichkeit, die eigene Sexualität in jedem Alter ausleben zu können (vgl. WHO-Regionalbüro für Europa 2011). Dabei solle Sexualität im Alter nicht als besonders oder krankhaft betitelt werden, sie müsse vielmehr als natürlich und dazugehörig zum Älterwerden gelten und wie jedes andere Grundbedürfnis auch behandelt werden (ZettlWiedner 2011).

Die Bedeutsamkeit des Themas der sexuellen Gesundheit in der Altenpflege basiert auf folgenden Überlegungen: Das Statistische Bundesamt (2015) schätzt, dass bis zum Jahr 2030 die Anzahl der Pflegebedürftigen ab 65 Jahren auf 3 Millionen ansteigen wird. Demzufolge ist zu erwarten, dass dadurch auch die Zahl der professionell Gepflegten im häuslichen 
Umfeld oder in Heimen steigen wird. Für diese Menschen wird das Altenheim zu einem neuen Zuhause, einem Ort an dem gewohnt, gegessen, geschlafen, gearbeitet, geliebt und gestorben wird (Michelchen 2008). Altenpflegende beschäftigen sich somit mit vielen, besser gesagt mit allen Aspekten im Leben eines Bewohnenden bzw. Pflegebedürftigen (vgl. Michelchen 2008).

Der Wunsch, seine Sexualität ausleben zu können, bleibt bis ins hohe Alter bestehen (Bucher et al. 2003; Buchner et al. 2011). Durch ein striktes zeitlich und räumlich organisiertes Umfeld im Heim ist das aktive Ausleben von Sexualität für Bewohnende womöglich schwer zu realisieren (vgl. Meudt 2006). Fehlende Intim- und Privatsphäre, Mehrbettzimmer und wenig privater Freiraum behindern Bewohnende, sich sexuell verwirklichen zu können (Meudt 2006). Dadurch werden vor allem im stationären Bereich die Intimität und der Umgang mit Sexualität in der Bewohnendenversorgung zu einer Herausforderung (Meudt 2006). Sexuell aktive Heimbewohnende stellen für das Pflegepersonal eine potenzielle Herausforderung dar, sowohl auf professioneller als auch auf persönlicher Ebene (vgl. Grond 2011). Laut Grond (2011) könne das offene Ansprechen von Sexualität Pflegenden und Betroffenen helfen, Vertrauen aufzubauen und gleichsam persönliche Grenzen zu setzen.

Der Autor Gunnar Michelchen (2008) versucht die Begriffe Intimität und Sexualität zu differenzieren, denn ihm zufolge werden sie häufig gleichgesetzt verwendet. Die lateinische Übersetzung des Begriffes „Intimität“ würde treffender mit „Innigkeit“ oder „Vertrautheit“ umschrieben werden (Michelchen 2008). In der umgangssprachlichen Auslegung des Wortes „Intimität“ fänden sich vor allem körperliche Nähe und sexuelle Kontakte (vgl. Michelchen 2008). Abzugrenzen davon sind die Begriffe der Privat- und Intimsphäre. Während die Privatsphäre sich eher auf einen Raum bezieht, der der Öffentlichkeit verborgen bleiben sollte, bezieht sich die Intimsphäre auf Bereiche, die sich mit den innersten persönlichsten Gedanken und Gefühlen befassen (Michelchen 2008). Allenfalls wird auserwählten Personen ein Einblick in diese gewährt. Laut Michelchen (2008) würde jede pflegerische Tätigkeit einen Eingriff in die Intimsphäre darstellen, worüber sich jeder Pflegende im Klaren sein sollte. In vielen deutschen Altenheimen basiert die Pflege der Bewohnenden auf dem Konzept der „13 Aktivitäten und existenziellen Erfahrungen des Lebens“ (AEDL), welche die Pflegewissenschaftlerin Monika Krohwinkel entwarf (vgl. Michelchen 2008). Sie sollen beinahe alle für einen Menschen notwendigen Tätigkeiten und Bedürfnisse umfassen. Zu diesen gehören unter anderem: „kommunizieren, sich bewegen, sich pflegen, ausscheiden, essen und trinken, sich kleiden, schlafen, für eine sichere Umgebung sorgen und sich als Mann oder Frau fühlen“ (vgl. Michelchen 2008). Viele dieser genannten Tätigkeiten verlangen zum Ausleben eine spezielle Intimität. Doch mit zunehmendem Alter wird auch 
bei diesen intimen Handlungen Hilfe benötigt. Dennoch ändert sich der Wunsch nach Intimität auch mit zunehmender Pflegebedürftigkeit nicht. Der Eingriff in die Intimsphäre sei besonders im stationären Bereich bedeutsam, da hier die Pflege von nicht vertrauten, fremden Pflegenden übernommen werde. Altenpflegende sollen sich des Eingriffs in die Intimsphäre und der damit verbundene Prozesse für den Betroffenen bewusst sein (Michelchen 2008).

\subsection{Literaturübersicht}

Im folgenden Kapitel wird auf die für die vorliegende Arbeit wesentliche Forschungsliteratur zu den Themen Sexualität und Pflege eingegangen. Im Rahmen der Literaturrecherche wurde deutlich, dass es nur wenig Fachliteratur und aktuelle Forschung zu den Bereichen Sexualität in der Altenpflege und -ausbildung in Deutschland gibt. Zum Thema sexuelle Gesundheit in Bezug auf die Altenpflegeausbildung und den Umgang von Auszubildenden mit der Sexualität der Bewohnenden lagen zum Zeitpunkt der Erhebung keine deutschen Studien vor.

In dieser Arbeit wird zum Teil auf ältere und nicht-deutschsprachige Literatur zurückgegriffen. Auch zeigte sich, dass Studien zum Thema Sexualität und Pflege häufig nur eine kleine Stichprobe betrachteten. Grund hierfür ist möglicherweise, dass Sexualität im Alter als Tabuthema gilt bzw. als solches behandelt wird (vgl. Grond 2001; Michelchen 2008; Meudt 2006). Mittlerweile allerdings wird die Sexualität älterer Menschen nicht selten in der Populärliteratur und auch im Zusammenhang mit (Alten)pflege erwähnt. Auch das Internet bildet eine große Austauschfläche für diverse Aspekte der Thematik Sexualität im Alter. Körperliche Veränderungen im Alter werden hier angesprochen, einschränkende medikamentöse Therapien erläutert und Erkrankungen wie bspw. Demenz, die mit einer sexuellen Enthemmung einhergehen, werden benannt; das Informationsspektrum ist breit gefächert. Hingegen gibt es nur wenige wissenschaftliche Studien zur Bedeutung der Alterssexualität im Arbeitsalltag der Altenpflegenden.

Michelchen (2008) zufolge betreibt die Gesellschaft eine Verdrängung des Wissens über die sexuelle Aktivität älterer Menschen. Für viele erscheint die Selbstverständlichkeit, dass ältere Menschen sexuell aktiv sind, unwirklich (Michelchen 2008). Durch die Gesellschaft finde eine Verleugnung der Sexualität älterer Menschen statt, indem diese als asexuell betrachtet würden (Zettl 2000). Gründe für die gesellschaftliche Verdrängung der Sexualität älterer Menschen seien unter anderem die öffentliche, aber auch private Tabuisierung der Thematik. Sexualität als Grundbedürfnis und die Natürlichkeit der Thematik seien auch im Bereich der Altenpflege vorurteilsbehaftet und von Unsicherheit geprägt (Michelchen 2008). Doch 
gerade in der Altenpflege bedürfe es einer besonderen Offenheit gegenüber Sexualität (Grond 2011). Das Thema Sexualität solle Teil der Pflegeanamnese sein (Grond 2011). Da sexuelle Zufriedenheit auch im Alten- oder Pflegeheim Lebensqualität auszeichnet, sollte die empfundene Scham überwunden werden (Grond 2001). Offenheit sei nur möglich, wenn Pflegende sich Fragen zu Selbsterfahrung und Selbstkenntnis stellen und reflektiert an die Thematik herantreten (Grond 2001). Der Verein Pro Familia bietet unter dem Titel „Sexualität - (K)ein Thema in der Altenpflege?“ Fortbildungsmöglichkeiten für Pflegende zu den Themen Sexualität und Altenpflege an (vgl. Wendland 2013). Studienergebnisse bezüglich der Teilnehmerzahlen und dem Einfluss von Fortbildungen auf den Pflegealltag liegen zum jetzigen Zeitpunkt keine vor. In einem Magazin des Vereins schreibt Wendland (2013) über die Natürlichkeit von Sexualität im Alter, die Notwendigkeit von Fortbildungen für Altenpflegende sowie die erforderliche Selbstreflexion, um mit sexuell aktiven Heimbewohnenden/älteren Menschen umzugehen. Weiterhin werden die Herausforderungen, Platz für Sexualität in der Pflegesituation - sowohl auf zeitlicher als auch personeller Ebene zu schaffen - angesprochen (vgl. Wendland 2013).

Der Wunsch nach einer aktiven Sexualität bleibt auch noch im hohen Alter bestehen (vgl. Bucher et al. 2003; Buchner et al. 2011; Grond 2001; Michelchen 2008). In der Fragebogenstudie „Sexualität in der zweiten Lebenshälfte“, die in der deutschsprachigen Schweiz durchgeführt wurde, wurden 857 Frauen und 641 Männer von 45 bis 91 Jahren zum Thema Sexualität befragt (Bucher et al. 2003). Obwohl eine Diskrepanz zwischen sexuellem Interesse und dem aktiven Ausleben von Sexualität besteht, zeigen die Ergebnisse der Studie, dass Sexualität auch im hohen Alter noch relevant bleibt. Ein wesentlicher Faktor für das Fortbestehen einer aktiven Sexualität im höheren Alter ist unter anderem die Bedeutsamkeit von Sexualität in jüngeren Jahren sowie ein aktiver Lebensstil (Bucher et al. 2003). Die Pilotstudie „Sexualität im Alter“ der Helios Klink Berching befasste sich ebenfalls mit der Sexualität von Menschen über 65 Jahren (Buchner et al. 2011). Im Rahmen einer Interviewstudie in den Jahren 2009 und 2010 wurden 50 Männer und Frauen zu ihrer Sexualität befragt. Mittels Screening-Verfahren wurden vorher die Erkrankungen Demenz und Depression ausgeschlossen. Teilnehmende wurden zu der Veränderung ihrer Sexualität im partnerschaftlichen Kontext befragt. Demnach werde der Geschlechtsverkehr an sich zwar seltener, aber er verändere sich, werde schöner und Zärtlichkeiten würden an Bedeutung gewinnen. Gründe für sexuelle Enthaltsamkeit sind unter anderem Libidoverlust $(\mathrm{n}=8)$, Erkrankungen/Pflegebedürftigkeit $(\mathrm{n}=7)$ oder das Versterben $(\mathrm{n}=4)$ des Partners sowie eigene Erkrankungen ( $n=3$ ) (Buchner et al. 2011). Die Zusammenschau der Ergebnisse zeigt, dass Sexualität auch im hohen Alter Bestandteil des Lebens ist und dass 
daher das Wahrnehmen von alten Menschen als Wesen mit sexuellen Gefühlen und Wünschen wichtig sei. Die Autoren diskutieren, dass im Hinblick auf diese Erkenntnis, Sexualität und sexuelle Aktivität mehr Beachtung in der Anamnese und Therapieplanung in der Altenpflege finden sollte (Buchner et al. 2011).

Lewis und Bor (1994) fanden in ihrer Fragebogenstudie heraus, dass von 161 befragten Krankenpflegenden in ausgewählten Londoner Krankenhäusern die Mehrzahl (86,9\%) es als Teil ihrer pflegerischen Tätigkeit ansah, eine beratende Funktion im Bereich Sexualität zu übernehmen. Im Rahmen ihrer pflegerischen Grundausbildung wurden 68,1\% der Befragten im Bereich Sexualität geschult; knapp ein Fünftel (18,8\%) wurde explizit das Erheben einer Sexualanamnese gelehrt. Die Mehrheit (78,5\%) des befragten Krankenpflegepersonals fühlte sich gut genug ausgebildet, um mit Patientenangelegenheiten bezüglich Sexualität umzugehen (Lewis und Bor 1994). Auch die Arbeitserfahrung und das Alter der Pflegenden schienen einen Einfluss auf die Einstellung gegenüber Alterssexualität zu haben (vgl. Bouman et al. 2007; Sieren 2012). So zeigte englisches Pflegepersonal in Pflegeheimen in Nottingham mit einer Arbeitserfahrung unter fünf Jahren eine deutlich restriktivere und negativere Einstellung gegenüber Alterssexualität als Personal mit mehr Arbeitserfahrung (Bouman et al. 2007). In einer Studie, die in norddeutschen Pflegeheimen durchgeführt wurde, erwiesen sich ältere Fachkräfte als toleranter gegenüber der Sexualität der Bewohnenden (Sieren 2012). Die jüngeren Teilnehmenden gaben an, während der Ausbildung nicht ausreichend auf die Thematik Alterssexualität vorbereitet worden zu sein. So käme es erst durch das Sammeln eigener Erfahrungen und den Austausch mit anderen Mitarbeitenden zu Kenntnissen in diesem Bereich (Sieren 2012).

Für Pflegende könne es schwierig sein, hilflose und multimorbide Bewohnende mit der Vorstellung eines noch vorhandenen Bedürfnisses nach Sexualität und Zärtlichkeit in Einklang zu bringen (van der Vight-Klußmann 2014). Bouman et al. (2007) vermuteten, dass Pflegende, die vorwiegend mit älteren, multimorbiden Personen arbeiteten, gegenüber dem Thema Alterssexualität restriktiver eingestellt waren, als Pflegende, die vergleichsweise gesunde Personen betreuten. Ekel, Scham und persönliche Grenzüberschreitungen könnten die Offenheit gegenüber der Sexualität erschweren und könnte Pflegende vor verschiedene Herausforderungen stellen (vgl. van der Vight-Klußmann 2014; Kleinevers 2004). So könne es mit einer Zunahme von Nähe und Vertrautheit zu gegenseitig empfundenen Sympathien und auch zu erotischen Gefühlen zwischen Pflegendem und Gepflegtem kommen (Zettl 2000). Dies führe bei dem Behandelnden zu Verwirrung und Zwiespalt, denn hier stünden Lust, Schuld und Angst vor Kontrollverlust im Raum (Zettl 2000). Der Umgang mit Gefühlen von Angst, Schuld, Lust und Phantasien in der Patientenversorgung solle durch 
eine Reflexion der eigenen Situation besser geklärt werden (vgl. Zettl 2000; Kleinevers 2004). Dabei solle der Pflegende sich Fragen zu den eigenen, situativ-empfundenen Gefühlen stellen und diese auch mit Kollegen besprechen. Diese Herangehensweise sei allerdings aufgrund der Tabuisierung von Sexualität im Zusammenhang mit professioneller Pflege erschwert (Zettl 2000).

Zusammenfassend geht aus der Literatur hervor, dass Sexualität im Alter eher als Tabuthema behandelt wird (vgl. Grond 2011; Michelchen 2008; Meudt 2006). Der Wunsch nach Sexualität bleibt dennoch bis ins hohe Alter bestehen (vgl. Bucher et al. 2003; Buchner et al. 2011; Grond 2001; Michelchen 2008). Pflegende sollen sich reflektiert gegenüber ihrer eigenen Sexualität und der Sexualität anderer Menschen verhalten, um einen respektvollen Umgang mit Sexualität im Alter möglich zu machen (vgl. Grond 2001; Kleinevers 2004; Zettl 2000; Michelchen 2008). Die Ergebnisse einiger Studien deuten darauf hin, dass sowohl das Alter der Pflegenden als auch die Berufserfahrung einen Einfluss auf die Einstellung zur Sexualität von Gepflegten haben (vgl. Bouman et al. 2007; Sieren 2012). Eine Thematisierung von Sexualität im Rahmen der Ausbildung scheint hilfreich für den späteren Berufsalltag (vgl. Lewis und Bor 1994). Daher stellt sich nun die Frage, ob und in welchem Umfang das Thema Sexualität in der Altenpflegeausbildung behandelt wird.

\subsection{Ausbildung und Lernfelder in der Altenpflege}

Im weiteren Verlauf wird der Werdegang zum Altenpflegenden kurz skizziert und exemplarisch einige Lernfelder der Ausbildung beschrieben. Inwieweit diese in Bezug auf das Thema sexuelle Gesundheit ausgearbeitet werden, liegt im subjektiven Ermessen der Lehrenden. Ziel der Altenpflegeausbildung ist es, kompetent im Bereich der professionellen Pflege zu sein und somit allumfassend, nach individuellen Fähigkeiten und biographieorientiert, mit Menschen zusammenzuarbeiten (Niedersächsisches Kultusministerium 2003). Um in Deutschland eine Ausbildung zum Altenpflegenden zu beginnen, muss der Auszubildende bestimmte Zugangsvoraussetzungen erfüllen. $\mathrm{Zu}$ diesen gehören entweder ein Realschulabschluss oder eine andere abgeschlossene 10-Jährige allgemeine Schulausbildung. Bewerber mit einem Hauptschulabschluss müssen eine abgeschlossene Ausbildung in der Alten- oder Krankenpflegehilfe oder eine mindestens zweijährige anderweitige Berufsausbildung vorweisen. Außerdem wird ein Gesundheitszeugnis benötigt (Bundesministerium für Familie, Senioren, Frauen und Jugend 2016).

Die Ausbildung zum Altenpflegenden wird in theoretischer (2 100 Stunden) und praktischer (2 500 Stunden) Arbeit absolviert und dauert mindestens drei Jahre. Mit einem Arbeitsvertrag wird das Verhältnis zwischen Altenpflegeheim und Auszubildenden festgelegt. Zwischen 
Altenpflegeheim und -schule liegt ein Kooperationsvertrag vor (Bundesministerium für Familie, Senioren, Frauen und Jugend 2016).

Die Rahmenrichtlinien für den berufsbezogenen Lernbereich in der Berufsfachschule Altenpflege sehen unterschiedliche Lernfelder für Auszubildende der Altenpflege vor. Einige der für die Hauptthematik der vorliegenden Arbeit relevantesten Lernfelder werden an dieser Stelle kurz hervorgehoben.

Das Lernfeld „Lebenswelten und soziale Netzwerke alter Menschen beim altenpflegerischen Handeln berücksichtigen“ nennt unter dem Punkt „Unterstützung alter Menschen bei der Lebensgestaltung“" als direkten Inhalt unter anderem die Sexualität im Alter. Welche Aspekte hier jedoch im Detail behandelt werden, wird nicht genannt. Die Auszubildenden sollen reflektiert und respektvoll in allen Arbeitsbereichen handeln (Niedersächsisches Kultusministerium 2003).

In dem Lernfeld „Anleiten, beraten und Gespräche führen“ sollen die Auszubildenden lernen, Nähe und Distanz zu beachten und situationsangemessen zu reagieren. Ebenfalls soll ihnen die Möglichkeit gegeben werden, sich über eigene Rollenkonflikte, Unsicherheiten und Ängste auszutauschen (Niedersächsisches Kultusministerium 2003). In diesem Lernfeld werden sexuell konnotierte Situationen nicht explizit genannt, dennoch wäre es möglich, dass die Auszubildenden in diesem Ausbildungsblock Ressourcen für entsprechende Situationen erwerben.

Das Lernfeld „Altenpflege als Beruf ausüben“ beinhaltet zahlreiche Unterpunkte. Ein Teil davon, und auch Gegenstand der mündlichen Prüfung, ist das Thema „Gewalt in der Pflege“. Hier soll unter anderem auf die Themen „Macht in der Pflegehandlung, sexuelle Belästigung und gefährliche Pflege“ eingegangen werden. Ebenfalls wird das Thema „Spannung in der Pflege“ besprochen, welches Nähe- und Distanzkonflikte beinhaltet und ebenfalls Bestandteil der mündlichen Prüfung ist (Niedersächsisches Kultusministerium 2003).

Die genannten Lernfelder der Ausbildung bieten Inhalte an, die im erweiterten Sinne mit sexueller Gesundheit und Alterssexualität in Zusammenhang stehen und somit Berührungspunkte mit der Hauptthematik bilden. Die tatsächliche Ausarbeitung der Lernfelder und das Ausmaß an Inhalten zum Thema sexuelle Gesundheit bleiben jedoch der jeweiligen Schule oder den Lehrenden individuell überlassen. In der vorliegenden Studie wurde daher insbesondere auf die subjektive Präsenz des Bereichs Alterssexualität im Rahmen der Ausbildung und des Altenpflegealltags eingegangen. 


\subsection{Situation in den Pflegeheimen}

Um das Thema sexuelle Gesundheit in den Kontext der aktuellen Situation in Deutschland zu setzen, werden im Folgenden die Strukturen in deutschen Pflegeheimen beschrieben. Grundsätzlich werden drei verschiedene Heimtypen differenziert: das Altenwohnheim, das Altenheim und das Pflegeheim (vgl. Bundesministerium für Gesundheit 2014). Bewohnende im Altenwohnheim leben in eigenen Wohnungen mit Küche, können aber - falls gewünscht - mit anderen Bewohnenden Mahlzeiten einnehmen (vgl. Bundesministerium für Gesundheit 2014). Im Gegensatz dazu werden Bewohnende im Altenheim pflegerisch und hauswirtschaftlich unterstützt. Die Unterkunft besteht in der Regel aus kleinen Wohnungen oder Apartments. Bewohnende in Pflegeheimen werden allumfassend pflegerisch und hauswirtschaftlich versorgt. Häufig leben Bewohnende in Einzel- oder Doppelzimmern mit eigenen Möbeln (Bundesministerium für Gesundheit 2014). Das Statistische Bundesamt (2017) berichtet, dass es zum Jahresende 201513600 Pflegeheime in Deutschland gab, wovon bei 19\% ein Altenheim oder betreutes Wohnen angegliedert war. Im Durchschnitt fand eine Betreuung von 63 Pflegebedürftigen pro Heim statt (Statistisches Bundesamt 2017). Mitte Dezember 2015 befanden sich 759000 Personen in vollstationärer Dauerpflege. Der Hauptversorgungsschwerpunkt der Pflegeheime bildet die Betreuung älterer Menschen (s. Tabelle 1, Statistisches Bundesamt 2017).

Tabelle 1 Betreuungsschwerpunkte der Pflegeheime (basierend auf den Daten des Statistischen Bundesamtes 2017)

\begin{tabular}{ll}
\hline Versorgungsschwerpunkte: & Relative Anzahl der Heime [\%] \\
Betreuung älterer Menschen & 94 \\
Betreuung behinderter Menschen & 2 \\
Betreuung gerontopsychiatrischer oder & 2 \\
psychisch-erkrankter Menschen & \\
$\begin{array}{l}\text { Begleitung sterbender und schwerkranker } \\
\text { Menschen }\end{array}$ & 2 \\
\hline
\end{tabular}

Insgesamt waren laut dem Statistischen Bundesamt (2017) im Dezember 2015 rund 730000 Menschen in Pflegeheimen angestellt, wovon zwei Drittel Teil- und ein Drittel Vollzeit beschäftigt waren (s. Tabelle 2). 51100 Personen waren zum Zeitpunkt der Erhebung Auszubildende oder Umschulende und machten somit einen Anteil von 7\% der Beschäftigten aus. In der Altenpflege waren neben Auszubildenden der Altenpflege, examinierten Alten-, Gesundheits- und Krankenpflegern zusätzlich Praktikanten, Bundesfreiwilligendienst- 
leistende und Pflegehelfende beschäftigt. 34\% der Angestellten wiesen einen Abschluss als Altenpflegende vor, 10\% waren examinierte Gesundheits- und Krankenpflegende. Insgesamt waren 84\% der Beschäftigten weiblich (Statistisches Bundesamt 2017).

Tabelle 2 Arbeitsschwerpunkte der im Pflegeheim Beschäftigten ( $\mathrm{n}=730$ 000) (basierend auf den Daten des Statistischen Bundesamtes 2017)

\begin{tabular}{ll}
\hline Arbeitsschwerpunkte: & Relative Anzahl [\%] \\
Pflege und Betreuung & 64 \\
Hauswirtschaft & 16 \\
Haustechnik und sonstige Bereiche & 9 \\
„Betreuungsassistenz“ & 7 \\
Soziale Betreuung & 4 \\
\hline
\end{tabular}

\subsection{Pflegestatistik im Kontext des demographischen Wandels in Deutschland}

Die demographische Entwicklung in Deutschland wird dazu führen, dass ein großer Teil der älter werdenden Bevölkerung auf fremde Hilfe angewiesen ist, sowohl im häuslichen Umfeld durch Angehörige oder ambulante Pflegedienste als auch im Rahmen einer Heimversorgung (vgl. Statistisches Bundesamt 2015).

Das Statistische Bundesamt (2015) gab an, dass zum Jahresende 2013 von 81 Millionen Menschen in Deutschland 17\% der Generation 65 plus angehörten (circa jeder Fünfte). Schätzungsweise wird 2060 sogar jeder Dritte in Deutschland mindestens 65 Jahre alt sein (vgl. Statistisches Bundesamt 2015). Tabelle 3 stellt die Pflegebedürftigkeit in Abhängigkeit vom Alter dar. Als pflegebedürftig gelten laut dem Sozialgesetzbuch XI (SGB XI) Personen, die aller Voraussicht nach über einen mindestens sechsmonatigen Zeitraum hilfsbedürftig sein werden. Ursachen können körperliche, seelische oder geistige Erkrankungen oder Behinderungen sein, die dazu führen, dass Betroffene erhebliche Einschränkungen in ihrem täglichen Leben aufweisen (Statistisches Bundesamt 2015). Statistisch gesehen steigt das Risiko eines Menschen, auf Pflege angewiesen zu sein, je älter er wird (vgl. Statistisches Bundesamt 2015, s. Tabelle 3). 
Tabelle 3 Anzahl der Pflegebedürftigen zum Jahresende 2013 (basierend auf den Daten des Statistischen Bundesamtes 2015)

\begin{tabular}{ll}
\hline Altersgruppe [Jahre] & Pflegebedürftigkeit [\%] \\
$65-69$ & 3 \\
$80-84$ & 21 \\
$\mathrm{ab} 90$ & 64 \\
\hline
\end{tabular}

Zum Jahresende 2013 betrug die Anzahl der Pflegebedürftigen 2,2 Millionen (Statistisches Bundesamt 2015). Zwei Jahre später war die Anzahl bereits auf 2,9 Millionen Menschen gestiegen (vgl. Statistisches Bundesamt 2017). Das Statistische Bundesamt differenziert die Versorgung pflegebedürftiger Menschen. So werden Pflegebedürftige bspw. zuhause durch Angehörige und/oder ambulante Pflegedienste oder im Heim versorgt. Die Daten zur Versorgungsart der Pflegebedürftigen des Statistischen Bundesamtes aus den Jahren 2013 und 2015 sind in Abbildung 1 und Abbildung 2 dargestellt (vgl. Statistisches Bundesamt 2015; Statistisches Bundesamt 2017). Von 2,2 Millionen wurden 708000 (33\%) der Pflegebedürftigen ab 65 Jahre zum Jahresende 2013 vollstationär im Heim versorgt (Statistisches Bundesamt 2015). Im Jahr 2015 waren es von 2,9 Millionen Pflegebedürftigen 783000 (27\%) (Statistisches Bundesamt 2017).

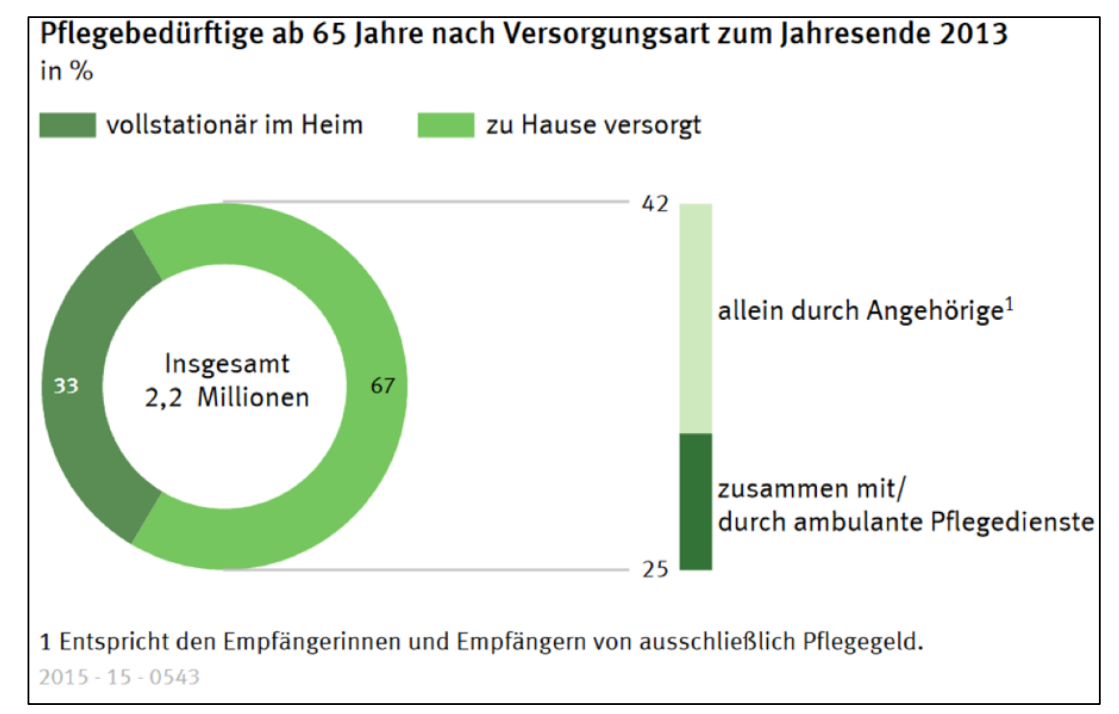

Abbildung 1 Pflegebedürftige ab 65 Jahre nach Versorgungsart zum Jahresende 2013, Schaubild 9.1 (Statistisches Bundesamt 2015) 


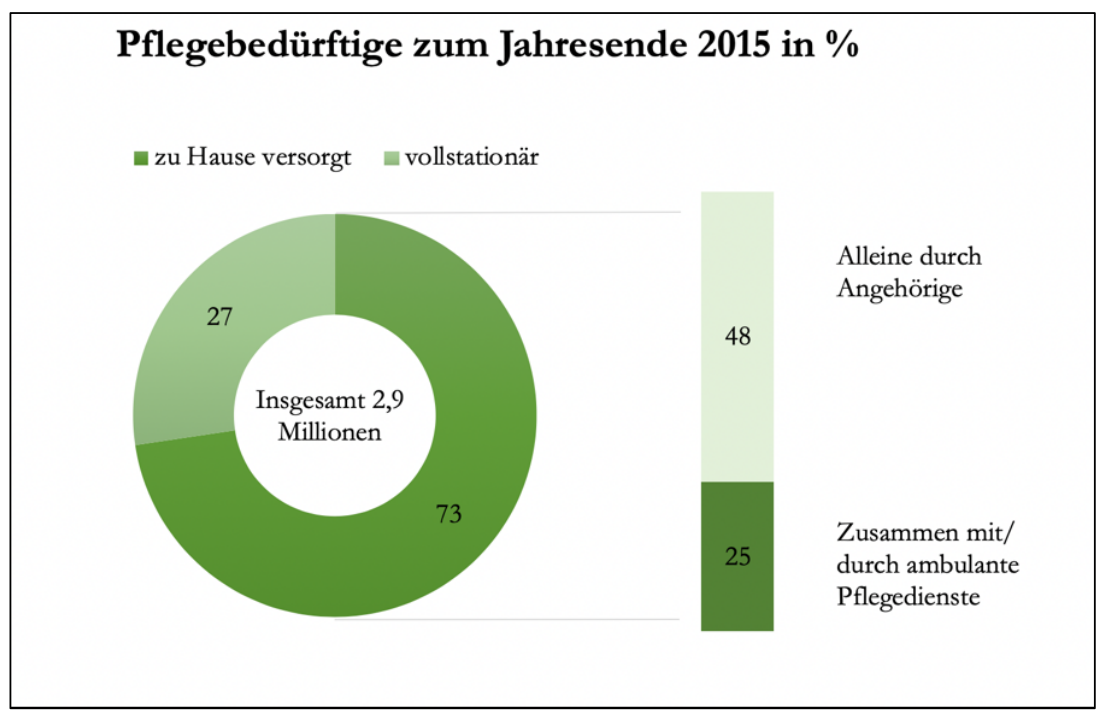

Abbildung 2 Pflegebedürftige zum Jahresende 2015 (basierend auf Daten des Statistischen Bundesamtes 2017)

Laut dem Statistischen Bundesamt (2015) hatten Männer und Frauen bis zu ihrem 75. Lebensjahr einen ähnlichen Bedarf an Pflege. Jedoch lag der Anteil der pflegebedürftigen 85bis 89-Jährigen Frauen bei 42\% und bei gleichaltrigen Männern bei 30\% (Stand 2013). Demnach werden mit steigendem Alter zunehmend Frauen pflegebedürftig. Eine mögliche Erklärung hierfür ist, dass Frauen eine im Schnitt höhere Lebenserwartung als Männer aufweisen (Statistisches Bundesamt 2015). In der Mehrzahl sind Frauen durch Verwitwung alleinstehend. So waren 51\% der alleinstehenden Frauen zwischen 65 und 69 Jahren und 87\% ab einem Alter von 85 Jahren verwitwet (vgl. Statistisches Bundesamt 2015). Dies führt dazu, dass der weibliche Anteil Alleinstehender im höheren Alter größer und ihr Bedarf an Pflegeleistungen höher ist. Bei Männern hingegen erfolgte häufiger eine Versorgung zuhause durch die Partnerin. Wenn hier keine Antragstellung auf Leistungen erfolgte, wurden diese Männer auch nicht von der Pflegestatistik erfasst. Dadurch blieb die Anzahl der pflegebedürftigen Männer nach dem SGB XI geringer als die Anzahl der pflegebedürftigen Frauen (Statistisches Bundesamt 2015).

Die demographische Entwicklung in Deutschland führt dazu, dass die Heimunterbringung einen bedeutsamen Anteil in der Versorgung pflegebedürftiger Menschen darstellt (vgl. Statistisches Bundesamt 2015; Statistisches Bundesamt 2017). Es ist davon auszugehen, dass von diesen im Heim versorgten Menschen ein Anteil noch sexuell aktiv ist. In Anbetracht dieser Tatsache sollte sexuelle Gesundheit in der Altenpflege jetzt und auch zukünftig thematisiert werden. 


\subsection{Fragestellungen und Zielsetzung der Arbeit}

Ziel der vorliegenden Arbeit war es, anhand einer prospektiven Fragebogenstudie die Aktualität und den Stellenwert des Themas sexuelle Gesundheit in der Altenpflegeausbildung und im Pflegealltag zu erheben. Die Studie konzentrierte sich insbesondere auf auszubildende und examinierte Altenpflegende. Ebenso erfolgte im Rahmen der Datenerhebung eine Befragung der Lehrenden, auf die in dieser Dissertation nur begrenzt eingegangen wird (s. Kapitel 2.3).

Aufgrund der Vielzahl der Items (95) des Fragebogens wurden die Forschungsfragen in die untenstehenden fünf Hauptfragen (Q 1 - 5) strukturiert. Im Rahmen der vorliegenden Studie galt es, die aktuelle Situation deskriptiv anhand einer möglichst großen Stichprobe darzustellen, um für spätere Studien mögliche Zusammenhangshypothesen abzuleiten. Im Folgenden werden die Hauptfragen Q1 - 5 vorgestellt.

\section{Q 1: Welchen Stellenwert hat das Thema Sexualität und sexuelle Gesundheit in der} Altenpflegeausbildung?

Die Fragestellung Q 1 befasst sich zum einen mit der Behandlung des Themas Alterssexualität in der Altenpflegeausbildung aus Sicht der befragten examinierten und auszubildenden Altenpflegenden (Item 2.1 - 2.4). Zum anderen wurden einige Ansichten der Lehrenden zum Thema Alterssexualität als Ausbildungsinhalt untersucht.

\section{Q 2: Welchen Stellenwert hat das Thema Sexualität und sexuelle Gesundheit im Pflegealltag?}

Unter der Fragestellung Q 2 werden verschiedene Aspekte und Berührungspunkte von Sexualität und sexueller Gesundheit im Pflege- bzw. Arbeitsalltag der Studienteilnehmenden zusammengefasst. Die Zusammenschau der Items dient dazu, einen Gesamteindruck des subjektiven Stellenwertes des Themas sexuelle Gesundheit im Altenpflegealltag zu erhalten. Unter anderem wurde nach dem Konzept der Sexualassistenz im Pflegealltag (Item 3.1 - 3.5) sowie nach Fortbildungsmöglichkeiten zu Alterssexualität und sexueller Gesundheit im Altenheim gefragt (Item 4.1 - 4.5). Die Studienteilnehmenden wurden zu sexuell konnotierten Situationen und erlebten Reaktionen zu sexueller Aktivität und Partnerschaften unter Heimbewohnenden befragt (Item 5.2 - 5.11, 7.1 - 7.12). Ein weiterer Aspekt von Q 2 bildete die Bereitstellung und Verfügbarkeit sexueller Hilfsmittel (Item 6.1 - 6.13), das ungestörte Ausleben von Sexualität (Item 6.14) sowie die Bedeutung von sexuell übertragbaren Erkrankungen und der ärztlichen Versorgung in der Altenpflege (Item 7.14 7.19). 
Q 3: Was ist der aktuelle Wissensstand in der Altenpflege zum Thema Sexualität und sexuelle Gesundheit?

Die Fragestellung Q 3 beschäftigt sich mit dem Wissensstand der Studienteilnehmenden zum Thema Sexualität. Anhand von vier Items (7.20 - 7.24) wurden Wissensfragen zu sexuellen Mythen, sexueller Orientierung und sexuellem Verhalten gestellt.

Q 4: Wie wird die eigene Sexualität, die Sexualität älterer Menschen und die Sexualität eigener Angehöriger wahrgenommen?

Im Rahmen der Fragestellung Q 4 wurden Fragen gestellt, die der Reflexion der eigenen Sexualität (Item 8.1, 8.6 - 8.13) und auch der Sexualität anderer Menschen (Item 8.3 - 8.4) dienen sollten. Weiterhin wurden den Studienteilnehmenden Fragen zum Ausleben von Sexualität im Alter (Item 8.2, 8.5) gestellt.

Q 5: Wie werden die Themen Sexualität im Elternhaus und Sexualaufklärung behandelt?

Die Fragestellung Q 5 befasst sich mit der Thematisierung von Sexualität im Elternhaus und im familiären Umfeld (Item 9.1 - 9.4). Weiterhin wurden Teilnehmende zu ihren Hauptaufklärungsquellen und -themen und dem subjektiven Grad der Aufgeklärtheit befragt (Item 9.5 - 9.7). 


\section{Methoden}

Im vorliegenden Kapitel folgt eine kurze Übersicht über das Ethikvotum sowie eine Angabe der Teilnahme- und Abbruchkriterien der Studie. Der Studienablauf des ersten und des zweiten Erhebungszeitraumes wird beschrieben. Weiterhin erfolgt die Vorstellung des Fragebogens für Auszubildende und Altenpflegende (Erhebungsinstrument).

\subsection{Ethikvotum}

Um die ethisch korrekte Durchführung der Studie zu gewährleisten, wurde ein Ethikvotum bei der Ethikkommission der Universitätsmedizin Göttingen beantragt (Antragsnummer 11/12/16). Die Studie sowie die Datenerhebung und -analyse wurden durch die Ethikkommission am 11.04.2017 genehmigt.

\subsection{Teilnahmekriterien}

Der folgende Abschnitt befasst sich mit den Ein- und Ausschlusskriterien der Studienteilnahme. Des Weiteren werden die Abbruchkriterien für den individuellen Teilnehmenden und die Gesamtheit der Studie vorgestellt.

\subsubsection{Ein- und Ausschlusskriterien}

\section{Einschlusskriterien}

Zur Teilnahme berechtigt waren Auszubildende und Lehrende der Altenpflegeschulen in Alfeld, Braunschweig, Duderstadt, Einbeck, Göttingen, Hannover, Hannoversch Münden, Hildesheim, Holzminden, Schladen und Uslar (die Auflistung erfolgt in alphabetischer Reihenfolge) sowie examinierte Altenpflegende und Altenpflegehelfende der ausgewählten Städte Hannover und Göttingen. Teilnehmende mussten mindestens 18 Jahre alt sein.

\section{Ausschlusskriterien}

Von der Studie ausgeschlossen wurden Personen unter 18 Jahren sowie nicht Auszubildende der oben genannten Altenpflegeschulen und nicht berufstätige Altenpflegende in Göttingen und Hannover. 


\subsubsection{Abbruchkriterien}

\section{Individuelle Abbruchkriterien}

Der individuelle Abbruch der Studie konnte jederzeit erfolgen. Als individueller Abbruch definiert ist die Nichtabgabe des Fragebogens nach erfolgtem Ausfüllen des Fragebogens sowie das Nichtausfüllen eines erhaltenen Fragebogens.

\section{Abbruchkriterien für die Gesamtstudie}

Es lagen keine Annahmen vor, für deren Eintritt der Abbruch der Studie die Folge gewesen wäre.

\subsection{Studienablauf}

Die Datenerhebung erfolgte im Rahmen einer prospektiven Fragebogenstudie in Göttingen (Niedersachsen) und in einem Radius von ca. $150 \mathrm{~km}$ um Göttingen. Befragt wurden examinierte Altenpflegende in Heimen, Auszubildende der Altenpflege sowie Lehrende an Altenpflegeschulen. Die Auswahl der Altenpflegeschulen erfolgte über die Internetpräsenz der Pflegekammer Niedersachsen (Pflegekammer Niedersachsen KdöR). Die Altenpflegeheime wurden über die Internetseite Heimverzeichnis.de (Heimverzeichnis $\mathrm{gGmbH}$ ) ausgewählt. Insgesamt wurden im ersten und zweiten Durchgang 147 Altenpflegeheime und 25 Altenpflegeschulen kontaktiert. Es nahmen 14 Altenheime und 11 Altenpflegeschulen an der Studie teil.

Initial bestand das Studiendesign aus zwei separaten Dissertationen mit zwei verschiedenen Fragebögen: (1) Auszubildende und Altenpflegende (Sexuelle Gesundheit in der Altenpflege) sowie (2) Lehrende (Sexuelle Gesundheit im didaktischen Kontext der Altenpflege). Die Arbeit zu den didaktischen Aspekten von Alterssexualität im Lehralltag wurde noch vor Studienbeginn durch eine weitere Doktorandin abgebrochen. Der Fragebogen für Lehrende wurde dennoch ausgegeben, um im Rahmen der durchgeführten Studie zumindest einige Aspekte dieser Thematik beleuchten zu können.

Aufgrund mangelnder Informationen $\mathrm{zu}$ den Themen sexuelle Gesundheit in der Altenpflegeausbildung und Altenpflege in deutschen Einrichtungen erfolgte im Rahmen der Studie die Erfassung eines Meinungsbildes anhand eines in der Arbeitsgruppe eigens dafür entworfenen Fragebogens. Angesichts der kontrovers diskutierten Thematik von Sexualität und Pflege war im Vorhinein nicht eindeutig absehbar, welche Größe die Stichprobe erreichen würde. Daher hatte sich die Arbeitsgruppe gegen eine Validierungsstudie und für eine, zu zwei Zeitpunkten erhobene, prospektive und deskriptive Studie entschieden. Ziel war es, so eine möglichst große Teilnehmerzahl zu erreichen. 
Die Erstellung des Fragebogens erfolgte primär mit dem Programm Microsoft Word Office 365. Nach Fertigstellung der Items wurden diese zur besseren Übersicht beim Ausfüllen und zur späteren Auswertung in die Umfragesoftware EvaSys als vollständiger Fragebogen übertragen.

Der Fragebogen wurde vor Studienbeginn im Rahmen eines Testdurchlaufs auf Inhalt und Verständnis geprüft. Er wurde von Studierenden der Medizin, examinierten Krankenpflegenden und Personen anderer Berufsgruppen ausgefüllt. Im Zuge der Pretests wurde der Fragebogen jedoch nicht von Auszubildenden oder examinierten Altenpflegenden bearbeitet; dies erfolgte erst während der Erhebungsphase. Während des Erstellungsprozesses und auch nach dem Testdurchlauf wurde der Fragebogen mit Mitarbeitern des Instituts für Statistik der Universitätsmedizin Göttingen besprochen.

Die Datenerhebung erfolgte in zwei Durchgängen, wovon der erste Durchgang im Juni und Juli 2017 stattfand und der zweite Durchgang von Juli bis November 2017 lief. Im ersten Durchgang wurden ausschließlich Altenpflegeheime und Altenpflegeschulen in und um Göttingen kontaktiert. Im zweiten Durchgang wurde der geographische Umkreis der Studie um ca. 150 km um Göttingen erweitert.

Im Zuge der Auswertung des ersten Durchgangs fiel auf, dass 16 Items bei der Übertragung der Items von Microsoft Word in die Umfragesoftware EvaSys nicht übernommen worden waren. Die Teilnehmenden des zweiten Durchgangs erhielten daher eine modifizierte Fragebogenversion, bei der die fehlenden 16 Items ergänzt wurden (Items 6.1 bis 6.16). Ansonsten war der Fragebogen identisch zu dem des ersten Durchgangs (s. Kapitel 2.4).

Die ausgewählten Einrichtungen wurden mit einem identischen Anschreiben postalisch kontaktiert. Ansprechpartner waren die Heim-, Pflegedienst- oder Schulleitungen. Ziel des Anschreibens war es, das Thema der Dissertation vorzustellen. Dem Schreiben war eine Kopie der Fragebögen angefügt, damit die Betreffenden eine genauere Vorstellung von der Studie und den zu erwartenden Fragen erhielten. Im Anschluss an die postalische Kontaktaufnahme erfolgte in den darauffolgenden Wochen eine telefonische Kontaktaufnahme. In einigen der Göttinger Altenpflegeheimen und -schulen erfolgten die erneute Vorstellung der Studie und das Austeilen der Fragebögen vor Ort in einem persönlichen Gespräch mit den Pflegedienst- oder Heimleitungen. Die geplante Vorstellung des Themas und der Fragebögen vor Ort gestaltete sich aus zeitlichen und logistischen Gründen als schwierig, weshalb vorwiegend eine postalische Übermittlung der Fragebögen stattfand.

Die Heim-, Pflegedienst- oder Schulleitungen trafen die Entscheidung über die Studienteilnahme der Einrichtung. Eine Zustimmung der Leitung verpflichtete den 
Einzelnen nicht zu der Studienteilnahme. Die individuelle Teilnahme der Auszubildenden, Lehrenden und Altenpflegenden erfolgte auf freiwilliger Basis.

Die von den Studienteilnehmenden ausgefüllten Fragebögen wurden in den Einrichtungen in fensterlosen Umschlägen oder Kartons an einem von den Leitungspersonen festgelegten Ort gesammelt. Anschließend wurden sie per Post oder durch Abholung an das Institut für Psychiatrie und Psychotherapie der Universitätsmedizin Göttingen zur nachfolgenden Auswertung gebracht. Das Auslesen der Fragebögen erfolgte mittels Einscannen in die Evaluations- und Umfragesoftware EvaSys in den Räumlichkeiten der Abteilung für Studium und Lehre der Universität Göttingen. Durch das o. g. Verfahren ist kein Rückschluss auf einzelne Personen möglich und die Anonymität der Teilnehmenden ist gewährleistet.

\subsubsection{Erster Durchgang (Juni - Juli 2017)}

Im ersten Durchgang waren 12 von 18 angefragten Altenpflegeheimen der Stadt Göttingen mit einer Studienteilnahme einverstanden. In den Altenpflegeheimen wurden 288 Fragebögen verteilt. Eine Rückgabe der ausgeteilten Fragebögen erfolgte durch 10 der 12 teilnehmenden Altenpflegeheime. Des Weiteren nahmen vier Schulen in den Städten Arnstadt, Göttingen und Schladen teil. Diese wurden mit einem dem der Heime identischen Anschreiben kontaktiert (12.06.2017). Es wurden 206 Fragebögen für die Auszubildenden sowie 29 Fragebögen für die Lehrenden verteilt, eine Rückgabe erfolgte durch alle vier Schulen.

Eine graphische Darstellung des Auswahlverfahrens des ersten Durchgangs ist der Abbildung 3 zu entnehmen. Insgesamt wurden 523 Fragebögen ausgegeben. Der gesamte Rücklauf des ersten Durchgangs betrug 220 Fragebögen für Auszubildende und Altenpflegende, sowie neun Fragebögen für Lehrende (Stand 27.07.2017). 


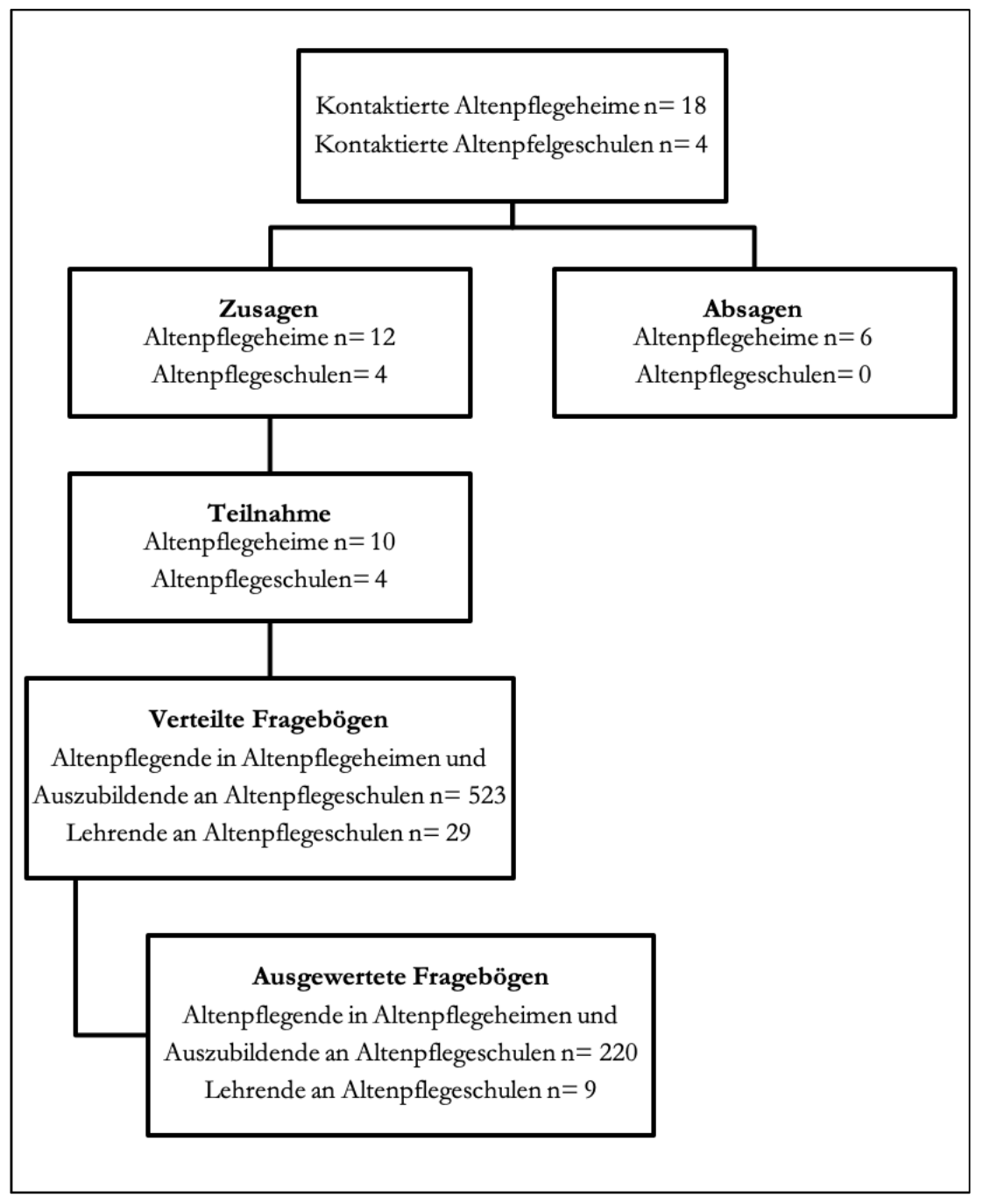

Abbildung 3 Erster Durchgang (Juli 2017)

\subsubsection{Zweiter Durchgang (Juli - November 2017)}

Die im zweiten Durchgang angeschriebenen Altenpflegeheime begrenzten sich auf die Landeshauptstadt Hannover. Hier wurden 130 Altenheime kontaktiert, wovon drei Altenheime aus Hannover der Teilnahme an der Studie zustimmten. Darüber hinaus nahm noch ein Göttinger Altenheim am zweiten Durchgang teil, welches den ersten Durchgang aus zeitlichen Gründen absagen musste. An die vier teilnehmenden Altenheime wurden 74 Fragebögen ausgegeben. Eine Rückgabe dieser erfolgte durch alle vier teilnehmenden Altenpflegeheime.

Der Umkreis der befragten Schulen wurde auf Städte im Radius von ca. $150 \mathrm{~km}$ um die Stadt Göttingen erweitert. Angeschrieben wurden Schulen in den Städten Alfeld, Braunschweig, Duderstadt, Einbeck, Göttingen, Hannover, Hannoversch Münden, Hildesheim, Holzminden, Schladen und Uslar (die Auflistung erfolgt in alphabetischer Reihenfolge). Von 23 kontaktierten Schulen stimmten insgesamt neun der Teilnahme an der Studie zu. Zwei dieser Schulen hatten bereits im ersten Durchgang mit anderen Klassen teilgenommen. An 
den Schulen wurden 551 Fragebögen für Auszubildende sowie 56 Fragebögen für Lehrende ausgegeben. Eine Rückgabe der ausgeteilten Bögen erfolgte durch alle neun teilnehmenden Altenpflegeschulen.

Eine graphische Darstellung des zweiten Durchgangs ist der Abbildung $4 \mathrm{zu}$ entnehmen. Insgesamt wurden 681 Fragebögen verteilt. Der gesamte Rücklauf des zweiten Durchgangs betrug 379 Fragebögen für Auszubildende und examinierte Altenpflegende, sowie 21 Fragebögen für Lehrende (Stand 16.11.2017).

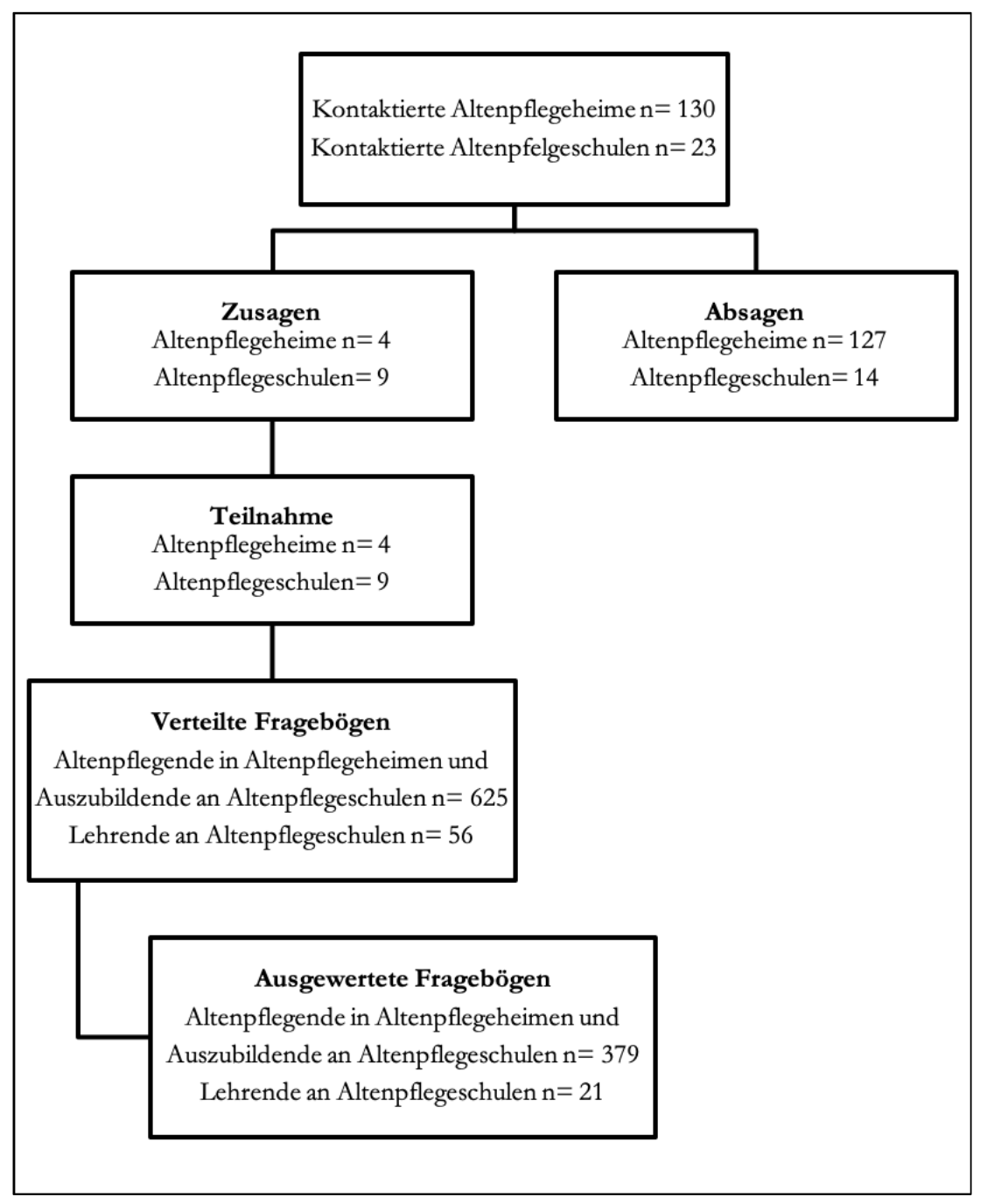

Abbildung 4 Zweiter Durchgang (November 2017)

\subsection{Fragebogen der Auszubildenden und Altenpflegenden}

Initiales Bestreben der Studie war die Erhebung eines Meinungsbildes zum Thema sexuelle Gesundheit in der Altenpflege und die Darstellung der Ergebnisse anhand einer deskriptiven Statistik. Die Auslegung des Fragebogens erfolgte mit ebendieser Zielsetzung. Der Fragebogen sowie die Mehrzahl der verwendeten Items wurden in der Arbeitsgruppe 
selbstständig entworfen. Einige Items entstammen der Forschungsliteratur und wurden im Fragebogen ergänzt (s. Kapitel 2.4.3). Die Items wurden mit dem Ziel entworfen, eine möglichst große Bandbreite an Informationen zum Thema sexuelle Gesundheit in der Altenpflege $\mathrm{zu}$ erlangen. Das Vorgehen und das Erhebungsinstrument wurde vor Studienbeginn 2017 mit Mitarbeitern des Instituts für Medizinische Statistik der Universitätsmedizin Göttingen besprochen.

Im folgenden Kapitel werden zum einen der Fragebogen der Auszubildenden und Altenpflegenden erläutert und zum anderen einzelne Items und Antwortoptionen exemplarisch vorgestellt. Der Fragebogen dient dazu, ein Meinungsbild der Einstellungen von Altenpflegenden, Auszubildenden und Lehrenden an Altenpflegeschulen zum Thema Sexualität und sexuelle Gesundheit in der Altenpflege zu erfassen. Die Fragebogenstudie erfolgte mit der Zielsetzung einer rein deskriptiven Auswertung. Weiterhin soll anhand des Fragebogens die Überprüfung der bereits in Kapitel 1.5 genannten Forschungsfragen erfolgen. Der vollständige Fragebogen ist dem Anhang zu entnehmen.

Für die vorliegende Studie wurden zwei Fragebögen in der Arbeitsgruppe neu entwickelt: ein Fragebogen für die Gruppe der Auszubildenden und examinierten Altenpflegenden sowie ein anderer für Lehrende an Altenpflegeschulen. Da sich die Arbeit überwiegend auf auszubildende und examinierte Altenpflegende bezieht, wird in diesem Kapitel der Schwerpunkt auf den Fragebogen dieser Gruppe gelegt. Orientiert wurde sich an allgemeinen Richtlinien und Empfehlungen zur Erstellung eines Fragebogens (vgl. Porst 2014; Krosnick und Presser 2009).

Der Fragebogen der Auszubildenden und Altenpflegenden bestand im ersten Durchgang aus 79 Items. Ergänzt wurden im zweiten Durchgang die Items 6.1 bis 6.16, die den neuen Teil 6 „Situation in meinem Berufsalltag“ bilden (s. Kapitel 2.3). Die endgültige Anzahl von Items des Fragebogens beträgt insgesamt 95. Davon entstammen 21 Items der Forschungsliteratur, 74 Items wurden von der Arbeitsgruppe entworfen.

Insgesamt lässt sich der Fragebogen in 11 Unterabschnitte einteilen, welche im Folgenden näher erörtert werden. Der einleitende Text zur Studie wurde im Fragebogen als Teil 1 gekennzeichnet und enthält Hinweise zum Ausfüllen des Fragebogens sowie einleitende Worte in die Studie (vgl. Porst 2014). Teil 11 dient am Schluss des Fragebogens für Anmerkungen der Teilnehmenden. Die zu beantwortenden Items wurden zur besseren Übersicht in 9 Kategorien thematisch sortiert (vgl. Krosnick und Presser 2009). Am Anfang des Fragebogens stehen Fragen, die das Hauptthema der Studie einleiten. Themenblöcke, die persönliche Fragen beinhalten (z. B. Fragen zur eigenen Sexualität und Offenheit in der Familie), wurden am Ende des Fragebogens platziert (vgl. Krosnick und Presser 2009). 
Es erfolgt eine Übersicht der Themenbereiche sowie in Klammern stehend die Anzahl der zugehörigen Items:

1) Hinweise zum Ausfüllen des Fragebogens (0)

2) Ausbildung (4)

3) Konzept der Sexualassistenz (5)

4) Fortbildungsmöglichkeiten am Arbeitsplatz (5)

5) Situation in meiner Einrichtung/meinem Wohnbereich (11)

6) Situation in meinem Berufsalltag (16)

7) Meinungsbild (24)

8) Fragen zur eigenen Sexualität (13)

9) Offenheit in der Familie (7)

10) Allgemeiner Teil (10)

11) Anmerkungen (0)

Im Folgenden werden die im Fragebogen verwendeten Antwortformate und exemplarisch einige Items vorgestellt. Weiterhin wird auf die Entwicklung des Fragebogens und die der Forschungsliteratur entstammenden Items eingegangen.

\subsubsection{Antwortformate}

In der vorliegenden Fragebogenstudie wurde vorwiegend ein geschlossenes Antwortformat gewählt, um die Antwortbereitschaft der Teilnehmenden $\mathrm{zu}$ erhöhen und die Ergebnisdarstellung im Rahmen der Auswertung zu vereinfachen (vgl. Porst 2014). Ausnahmen bildet der Teil 11 des Fragebogens, wo Teilnehmende die Möglichkeit hatten, Wünsche und Anregungen zu äußern sowie zwei Fragen, die im offenen Format zu beantworten waren (Item 6.10 und 6.12).

Offene Fragen:

6.10: Bereitstellung anderer als der hier genannten sexuellen Hilfsmittel in Ihrer Einrichtung:

Insgesamt erfolgte die Beantwortung der Items vorwiegend im Rahmen von Single- (Item 2.1 - 2.4, 3.1 - 3.5, 4.1 - 4.2, 4.5, 5.1 - 5.11, 6.1 - 6.8, 6.15 - 6.16, 7.1 - 7.3, 7.7 - 7.9, 7.13 7.18, 7.20 - 7.24, 8.1 - 8.5, 8.7 - 8.13, 9.1 - 9.5, 10.1 - 10.8, 10.10) und Multiple-Choice Optionen (Item 4.3 - 4.4, 6.9, 6.11, 6.13 - 6.14, 7.4 - 7.6, 7.10 - 7.12, 7.19, 8.6, 9.6 - 9.7, 10.9). Items, bei denen die Option einer Mehrfachnennung (Multiple-Choice) im Sinne einer 
Auflistung bestand, wurden alphabetisch aufgezeigt, um Antworttendenzen zu reduzieren und um eine möglichst neutrale, wertungsfreie Darstellung der Antwortmöglichkeiten zu bieten (vgl. Bogner und Landrock 2015).

Single-Choice:

3.1: Ist Ihnen das Konzept der Sexualassistenz bekannt? Ja

Nein

Weiß nicht

Multiple-Choice:

\begin{tabular}{|c|c|c|}
\hline 7.19: & $\begin{array}{l}\text { Mit welchen der folgenden sexuell übertragbaren } \\
\text { Erkrankungen hatten Sie in Ihrem Berufsalltag in } \\
\text { der Altenpflege schon zu tun? } \\
\text { Mehrfachnennungen möglich. }\end{array}$ & $\begin{array}{l}\text { Chlamydien } \\
\text { Gonorrhoe (Tripper) } \\
\text { Hepatitis B und C } \\
\text { Herpes genitales (HSV) } \\
\text { Human Immunodeficiency } \\
\text { Virus (HIV) } \\
\text { Humanes Papilloma Virus } \\
\text { (HPV- Feigwarzen) } \\
\text { Pilzinfektionen, z. B. } \\
\text { Scheidenpilze } \\
\text { Treponema pallidum } \\
\text { (Syphilis) }\end{array}$ \\
\hline
\end{tabular}

Bei der Verwendung endpunktverbalisierter Skalen erfolgte die Darstellung mittels fünfer Skalenbreite (Item 2.1 - 2.3, 7.14 - 7.15, 8.1, 8.7 - 8.12, 9.1 - 9.5). Franzen (2014) beschreibt, dass eine ungerade Anzahl an Antwortmöglichkeiten das Risiko der Akquieszenz - einer Tendenz zur inhaltsunabhängigen Zustimmung - verringert. Dennoch können ungerade Antwortmöglichkeiten die Interpretation von Ergebnissen erschweren, da Befragte, die keine Meinung zu einem Thema haben, eher zur Mitte tendieren (Franzen 2014). Weiterhin wurden die Endpunkte entsprechend der jeweiligen Frage modifiziert (bspw. 1= Trifft zu, 5= Trifft nicht zu; 1= Sehr offen, 5= Gar nicht offen), um das Verständnis der Antwortmöglichkeiten zu erleichtern und ggf. einer Ermüdung der Befragten vorzubeugen (vgl. Franzen 2014).

Endpunktverbalisierte Skalen:

\begin{tabular}{lll}
\hline 2.1: & $\begin{array}{l}\text { Ich fühle mich gut vorbereitet auf den Umgang mit verbal } \\
\text { und körperlich sexuell übergriffigen Verhalten von } \\
\text { Heimbewohnern. }\end{array}$ & $\begin{array}{l}\text { Trifft zu=1 } \\
\text { Trifft nicht zu=5 }\end{array}$ \\
\hline
\end{tabular}




$\begin{array}{lll}\text { 9.3: Wie offen haben meine Eltern mit mir über Sexualität } & \text { Sehr offen }=1 \\ \text { gesprochen? } & \text { Gar nicht offen }=5\end{array}$

\subsubsection{Entwicklung des Fragebogens}

Wie bereits in Kapitel 2.3 beschrieben, wurden die Items der Fragebögen vor Studienbeginn mehreren Personen verschiedener beruflicher Hintergründe, unter anderem auch Krankenpflegenden, vorgestellt und in einem Testdurchlauf auf Inhalt und Verständnis geprüft. Im Folgenden werden die durch den Testdurchlauf entstandenen Änderungen des Fragebogens aufgezeigt, welche aufgrund von Praktikabilität und Verständnis erfolgt waren.

Im Teil 1: „Hinweise zum Ausfüllen des Fragebogens“ wird auf den Verzicht einer geschlechtsspezifischen Differenzierung hingewiesen und somit der Übersichtlichkeit halber auf ein korrektes Gendern (bspw. Sexualassistentin/Sexualassistent, Heimbewohner/Heimbewohnerin) verzichtet (s. Fragebogen im Anhang). Weiterhin erfolgte in der vorliegenden Fragebogenversion eine Nummerierung und Re-Gruppierung der einzelnen Abschnitte und Items des Fragebogens (vgl. Krosnick und Presser 2009, s. Kapitel 2.4).

Wie bereits unter Kapitel 2.4.1 beschrieben, wurden die Multiple-Choice-Auflistungen alphabetisch aufgezeichnet, was in der Anfangsversion des Fragebogens nicht erfolgt war. Außerdem wurde bei den Items 7.4 - 7.6 und 7.10 - 7.12 die Antwortoption „Sonstiges“ mit der Möglichkeit einer Freitextangabe entfernt und auf eine geschlossene Frage mit Mehrfachnennung umgesetzt.

Initial war bei allen nominalskalierten Items nur eine dichotome Antwortmöglichkeit mit „Ja“ und „Nein“ gegeben. In der verwendeten Fragebogenversion wurde die zusätzliche Option „Weiß nicht“ hinzugefügt, um gegebenenfalls bei Unsicherheit und Meinungslosigkeit das Auslassen von Fragen zu reduzieren (Item 2.4, 3.1 - 3.5, 4.1, 4.2, 4.4, 6.8 - 6.9, 6.11, 6.13 - 6.15, 7.1 - 7.3, 7.7 - 7.9, 7.13, 7.16- 7.18, 7.20 - 7.24, 8.2 - 8.6).

\section{Erstversion:}

Ist Ihnen das Konzept der Sexualassistenz bekannt? Ja

Nein

Vorliegende Version:

\begin{tabular}{lll}
\hline 3.1: & Ist Ihnen das Konzept der Sexualassistenz bekannt? & Ja \\
& Nein \\
& Weiß nicht \\
\hline
\end{tabular}


Der fünfte Teil des Fragebogens befasste sich mit der Situation in der Einrichtung, beziehungsweise des Wohnbereichs. Die Items 5.2 und 5.3 wurden in der Anfangsfassung des Fragebogens anhand einer fünfstufigen endpunktverbalisierten Likert-Skala $(1=$ Nie, $5=$ Häufig) beantwortet. Zum besseren Verständnis und der Praktikabilität halber erfolgten eine Umformulierung der Items und eine Änderung des Antwortformats. In der vorliegenden Version wurde bewusst auf einen festen zeitlichen Abstand verzichtet, da vor allem das subjektive Empfinden des einzelnen Teilnehmenden erfasst werden sollte.

\section{Erstversion:}

Im Folgenden beantworten Sie bitte die Fragen nach der Häufigkeit ihrer Vorkommnisse.

Nie entspricht keinem Vorkommen, mit bäufig sind Vorkommnisse gemeint, die mehr als einmal im halben Jahr vorkommen.

\begin{tabular}{ll}
\hline Bestehende Partnerschaft zwischen & $1=$ Nie \\
Heimbewohner_innen & $5=$ Häufig \\
Neu gebildete Partnerschaft zwischen & $1=$ Nie \\
Heimbewohner_innen & $5=$ Häufig \\
\hline
\end{tabular}

Vorliegende Version:

\begin{tabular}{lll}
\hline 5.2: & Wie häufig bilden sich unter Heimbewohnern & Nie \\
& neue Paare? & Gelegentlich \\
& & Regelmäßig \\
5.3: & Wie häufig bilden sich Paare unter & Nie \\
& Heimbewohnern und Externen? & Gelegentlich \\
& Regelmäßig \\
\hline
\end{tabular}

Auch die Items 5.6 - 5.11 wurden in der Anfangsfassung anhand einer endpunktverbalisierten Likert-Skala ( $1=$ Nie, $5=$ Häufig) aufgelistet. Diese wurden in der Endversion des Fragebogens zur besseren Differenzierung auf eine fünfstufige Antwortmöglichkeit (s. unten) umgesetzt. Zudem wurde aus Gründen der leichteren Lesbarkeit die betreffende Gruppe markiert, um sie im Text hervorzuheben (bspw. Heimbewohner, Pflegende).

Erstversion:

$\begin{array}{ll}\text { Verbale sexuelle Übergriffe von } & \text { Nie=1 } \\ \text { Heimbewohner_innen auf Pflegende } & \text { Häufig=5 }\end{array}$


Vorliegende Version:

\begin{tabular}{lll}
\hline 5.6: & Verbale sexuelle Übergriffe von & Täglich=1 \\
& Heimbewohnern auf Pflegende: $z$. B. & Wöchentlich=2 \\
Aufforderungen zu sexuellen Handlungen & Monatlich=3 \\
& Seltener=4 \\
& Nie=5 \\
\hline
\end{tabular}

Item 5.7 bestand in der Erstversion aus drei Unterpunkten mit einer Freitextmöglichkeit im Feld Sonstiges. In der vorliegenden Version wurde die Antwortoption von Item 5.7 aus Gründen der Übersicht identisch zu denen von 5.6, 5.8 - 5.11 übernommen.

Erstversion:

$\begin{array}{ll}\text { Körperliche sexuelle Übergriffe durch } & \\ \text { Heimbewohner_innen } & \\ \text { An den Po fassen } & \text { Nie }=1 \\ & \text { Häufig }=5 \\ \text { An den Busen fassen } & \text { Nie }=1 \\ & \text { Häufig }=5 \\ \text { Sonstiges: } & \text { Nie }=1 \\ & \text { Häufig }=5\end{array}$

Vorliegende Version:

\begin{tabular}{lll}
\hline 5.7: & Körperliche sexuelle Übergriffe von & Täglich=1 \\
& Heimbewohnern auf Pflegende: z. B. in & Wöchentlich=2 \\
& den Schritt, Po oder Busen fassen & Monatlich=3 \\
& Seltener=4 \\
& Nie=5 \\
\hline
\end{tabular}

\subsubsection{Items der Forschungsliteratur}

An die Studie „SÄVIP - Studie zur ärztlichen Versorgung in Pflegeheimen“ angelehnt, wurde Item 7.17 entworfen (Hallauer et al. 2005). Unter anderem galt es im Rahmen der Studie SÄVIP herauszufinden, wer im Altenpflegeheim für die Bewohnenden Arztbesuche veranlasst: das Pflegepersonal, die Bewohnenden selbst oder ihre Angehörigen. Ebenso wurde den Personen, die den Arztbesuch initiierten, eine zeitliche Komponente zugeordnet: Nie/Selten/Zu gleichen Teilen/Meistens/Immer (vgl. Hallauer et al. 2005). Das daraus entwickelte Item 7.17 beschränkte sich bewusst auf die Fachrichtungen Gynäkologie und Urologie, da diese von der Arbeitsgruppe als für die sexuelle Gesundheit der Heimbewohnenden besonders relevant eingeschätzt wurden. 
Originalstudie:

\begin{tabular}{ll}
\hline Die Veranlassung von Arztbesuchen erfolgt & Nie \\
vornehmlich durch: Pflegepersonal/ & Selten \\
Heimbewohner/Angehörige & Zu gleichen Teilen \\
& Meistens \\
& Immer \\
\hline
\end{tabular}

Vorliegende Studie:

\begin{tabular}{lll}
\hline 7.17: & Wird von Seiten der Pflege darauf geachtet, dass es zu & Ja \\
& Vorsorgeuntersuchungen von Ärzten der Gynäkologie bzw. & Nein \\
& Urologie kommt? & Weiß nicht \\
\hline
\end{tabular}

Der Themenkomplex Q 3 befasst sich mit Fragen, die den Wissensstand der Teilnehmenden bezüglich des Sexualverhaltens von Männern und Frauen abfragen sollten. Die Items 7.20, 7.21, 7.23 und 7.24 stammen aus der Studie „Wie viel Sex braucht das Medizinstudium?“ (Turner et al. 2014). Die Items 7.21 und 7.23 wurden der Originalstudie unverändert entnommen. Item 7.20 wurde zum Verständnis der Teilnehmenden modifiziert: Klimakterium - Wechseljahre (vgl. Porst 2014). Item 7.24 wurde gekürzt und als geschlossene Frage formuliert:

Originalstudie:

Ist häufige Masturbation (ab 2x täglich) beim Jungen bzw. Körperlich: Mann körperlich und/oder psychisch schädlich? Wenn ja, Psychisch: welche Schäden können durch häufige Masturbation Weiß nicht hervorgerufen werden?

Vorliegende Studie:

\begin{tabular}{ll}
\hline 7.24: $\quad$ Ist häufiges Masturbieren psychisch schädlich? & Ja \\
& Nein \\
& Weiß nicht \\
\hline
\end{tabular}

Aus dem Englischen ins Deutsche übersetzt wurden die Items 8.1, 8.7, 8.12 und 8.13 des „Multidimensional Sexuality Questionnaire“ (Snell et al. 1993), ebenso die Items 8.8, 8.9, 8.10, 8.11 und 8.13 aus dem „Multidimensional Sexual Self-Concept Questionnaire“ von Snell et al. aus dem Jahr 1998 (Multidimensional Sexual Self-Concept Questionnaire). Aus Praktikabilitätsgründen wurde im Teil 8, der sich mit der eigenen Sexualität auseinandersetzt, das Antwortformat im Rahmen der Studie auf eine fünfstufige Likert-Skala ( $1=$ Trifft zu, 5= 
Trifft nicht zu) geändert. In der Erstfassung sollten Studienteilnehmende die Items 8.1 und 8.7 bis 8.13 mit den unten genannten Antwortoptionen A bis D abgleichen.

\section{Erstversion:}

Bitte beantworten Sie folgende Fragen nach diesem Schema:

A: überhaupt nicht charakteristisch für mich

B: weniger charakteristisch für mich

C: charakteristisch für mich

D: sehr charakteristisch für mich

\begin{tabular}{ll}
\hline Für meine eigene Sexualität bin ich zum Großteil selbst & A \\
verantwortlich. & B \\
& C \\
& D \\
\hline
\end{tabular}

Vorliegende Version:

\begin{tabular}{lll}
\hline 8.7: & Für meine eigene Sexualität bin ich zum Großteil selbst & Trifft zu=1 \\
verantwortlich. & Trifft nicht zu=5 \\
\hline
\end{tabular}

Die Items 9.1 bis 9.5 wurden der Dissertation „Psychosoziale Einflussfaktoren auf das weibliche Sexualerleben“ entnommen (vgl. Lehmann 2007). In der Originalstudie sollten Teilnehmende ein Kreuz auf einer endpunktverbalisierten Linie (bspw. Überhaupt nicht offen bis Sehr offen) setzen, welche keine festdefinierte Skalenbreite besaß. In der vorliegenden Studie wurde für die Beantwortung der Items 9.1 - 9.5 eine fünfer Skalenbreite gewählt. Die Items 9.6 und 9.7 wurden angelehnt an die Originalstudie entworfen und modifiziert.

Originalstudie:

2. Wie offen wurde in meiner Familie über Sexualität gesprochen?

überhaupt nicht offen sehr offen

Vorliegende Studie:

\begin{tabular}{lll}
\hline 9.2: & $\begin{array}{l}\text { Wie offen wurde in meiner Familie über Sexualität } \\
\text { gesprochen? }\end{array}$ & $\begin{array}{l}\text { Offen=1 } \\
\text { Gar nicht offen=5 }\end{array}$ \\
\hline
\end{tabular}


Originalstudie:

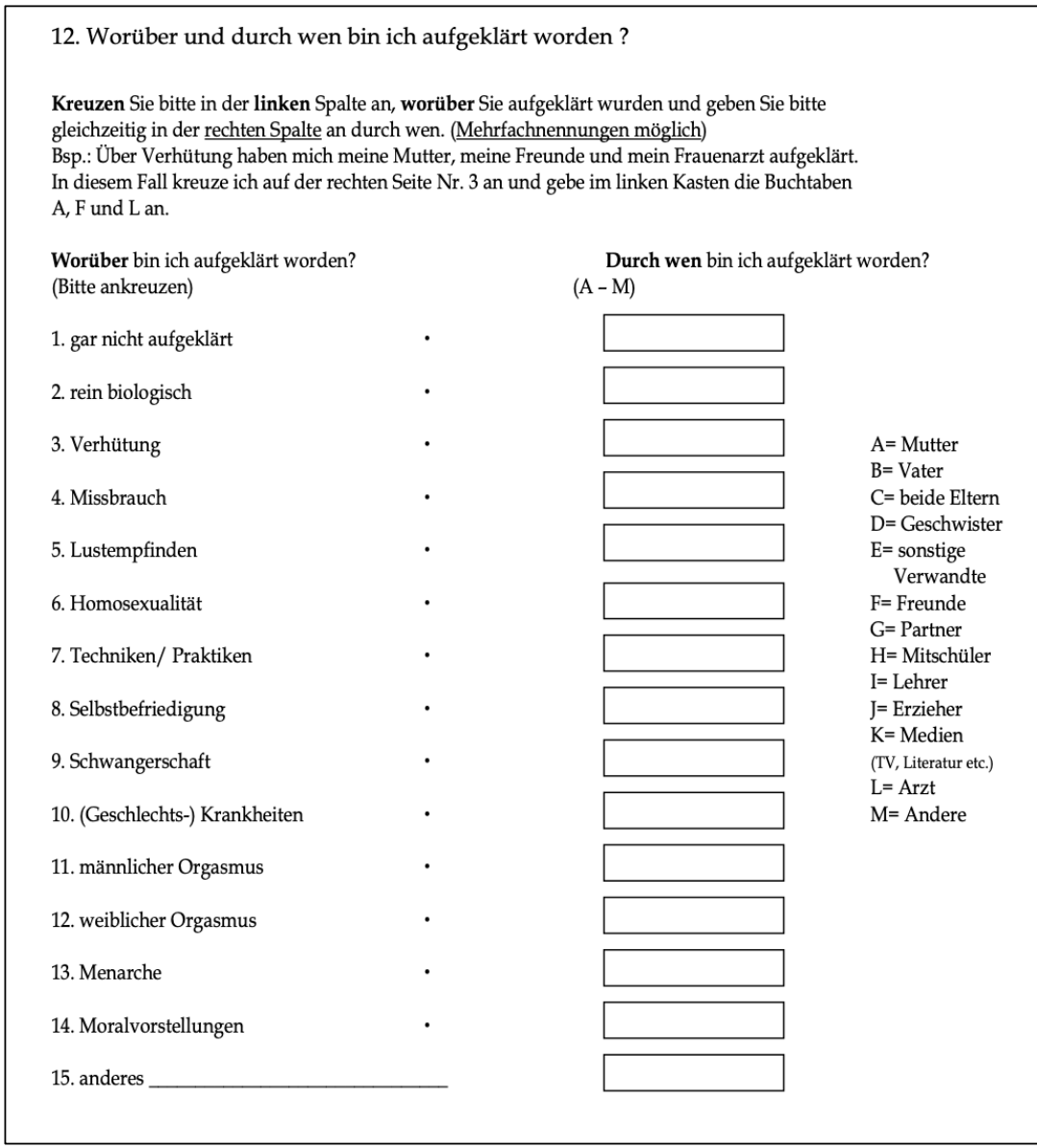

Vorliegende Studie:

9.6: $\quad$ Von wem bin ich hauptsächlich aufgeklärt worden? Mehrfachnennungen möglich.
Fernsehen

Freunde

Geschwister

Internet

Mutter

Schule

Vater

Zeitschriften

Gar nicht 


\begin{tabular}{|c|c|c|}
\hline 9.7: & $\begin{array}{l}\text { Über Folgendes bin ich von meinen Eltern } \\
\text { aufgeklärt worden: } \\
\text { Mehrfachnennungen möglich. }\end{array}$ & $\begin{array}{l}\text { Geburt } \\
\text { Geschlechtskrankheiten } \\
\text { Heterosexualität } \\
\text { Homosexualität } \\
\text { Monatliche Blutung } \\
\text { (Periode) } \\
\text { Schwangerschaft } \\
\text { Selbstbefriedigung } \\
\text { Sexuelle } \\
\text { Funktionsstörungen } \\
\text { Verhütung (Pille, Kondom, } \\
\text { Spirale..) } \\
\text { Gar nicht }\end{array}$ \\
\hline
\end{tabular}

\subsection{Datenauswertung}

Die Erhebung der Daten für die Fragebogenstudie sowie die Erfassung der Rohdaten erfolgte mittels der Evaluations- und Umfragesoftware EvaSys. Die damit gewonnenen Daten wurden mit Microsoft Excel 365 ausgewertet und deskriptiv mithilfe von Microsoft Word Office 365 dargestellt. Die Wahl des Auswertungsverfahrens wurde vor Studienbeginn mit Mitarbeitern des Instituts für Medizinische Statistik der Universitätsmedizin Göttingen im Rahmen der statistischen Beratung besprochen und befürwortet. Die Auswertung aller abgegebenen Fragebögen erfolgte unabhängig der Anzahl nicht beantworteter Items (Stichprobe, s. Kapitel 2.6). Hieraus ergibt sich pro Item eine unterschiedliche Stichprobe (n), welche im Folgenden separat aufgezeigt wird.

\subsection{Stichprobe}

Die Studie wurde anhand einer Stichprobe von 629 Teilnehmenden durchgeführt. An der prospektiven Fragebogenstudie nahmen insgesamt 599 Altenpflegende und Auszubildende sowie 30 Lehrende von Altenpflegeschulen teil. Es wurden insgesamt 1119 Fragebögen an Auszubildende und Altenpflegende verteilt, was bei einer Stichprobe von 599 einen Rücklauf von 53,53\% ergab. Für die Lehrenden wurden im ersten und zweiten Durchgang insgesamt 85 Fragebögen verteilt. Davon wurden 30 Fragebögen eingereicht, was einem Rücklauf von $35,3 \%$ entspricht.

Von n= 599 Teilnehmenden gaben 517 Personen Auskunft über ihren Ausbildungsstand. Zum Zeitpunkt der Datenerhebung waren 106 (20,5\%) Teilnehmende im ersten Ausbildungsjahr, 143 (27,7\%) Teilnehmende im zweiten Ausbildungsjahr und 171 (33,1\%) Teilnehmende im dritten Ausbildungsjahr. Eine abgeschlossene Berufsausbildung hatten 75 
$(14,5 \%)$ der Teilnehmenden. 22 Personen (4,3\%) hatten keine Ausbildung in der Altenpflege. 82 Personen trafen keine Aussage über ihren Ausbildungsstand.

Die Altersverteilung wurde in vorgeschriebenen Gruppen sortiert (s. Tabelle 4). Eine Auskunft über ihr Alter gaben 534 (89,1\%) Teilnehmende. Mit einem Anteil von 39,3\% ( $n=210)$ war der Großteil der Befragten in der Altersgruppe <25 Jahre. Eine Auskunft zu ihrem Geschlecht machten 418 (69,7\%) Personen. 117 (28\%) der Befragten waren männlich, 283 $(67,7 \%)$ waren weiblich und 18 (4,3\%) Teilnehmende fühlten sich einem anderen Geschlecht zugehörig. Tabelle 4 zeigt die Einordnung der Geschlechter zu den Altersgruppen, unter Berücksichtigung der fehlenden Angaben von 65 Teilnehmenden zum Alter und der NichtAngabe des Geschlechts von 181 Befragten.

Tabelle 4 Alter und Geschlecht der Teilnehmenden

\begin{tabular}{lllll}
\hline $\begin{array}{l}\text { Altersgruppe } \\
\text { Jahre] }\end{array}$ & $\begin{array}{l}\text { Anzahl } n \\
(\%)\end{array}$ & $\begin{array}{l}\text { Männlich } n \\
(\%)\end{array}$ & Weiblich $n(\%)$ & $\begin{array}{l}\text { Andere Zugehörigkeit } n \\
(\%)\end{array}$ \\
$<25$ & $210(39,3)$ & $44(40,4)$ & $116(42,4)$ & $2(18,2)$ \\
$25-35$ & $140(26,2)$ & $38(34,9)$ & $64(23,3)$ & $4(36,4)$ \\
$35-45$ & $72(13,5)$ & $15(13,8)$ & $31(11,3)$ & $0(0)$ \\
$45-55$ & $67(12,5)$ & $6(5,5)$ & $49(17,8)$ & $2(18,2)$ \\
$55-60$ & $19(3,6)$ & $1(0,9)$ & $8(2,9)$ & $0(0)$ \\
$>60$ & $26(4,9)$ & $5(4,6)$ & $7(2,5)$ & $3(27,3)$ \\
Gesamt & 534 & 109 & 275 & 11 \\
\hline
\end{tabular}




\section{Ergebnisse}

Die vorliegende Studie befasst sich mit der subjektiven Einschätžng von Auszubildenden und Altenpflegenden im Hinblick auf das Thema sexuelle Gesundheit in der Altenpflege. Insbesondere Fragen zu Häufigkeiten spiegeln daher nicht zwangsläufig die reale Situation in der Altenpflege, sondern vielmehr die subjektiven Vorkommnisse von Ereignissen wider. Ziel der Arbeit war es, das erfasste Meinungsbild im Rahmen einer deskriptiven Statistik darzustellen.

\subsection{Q 1: Welchen Stellenwert hat das Thema Sexualität und sexuelle Gesundheit in der Altenpflegeausbildung?}

Die Items 2.1 - 2.4 dienten dazu, den Stellenwert des Themas Alterssexualität in der Altenpflegeausbildung zu erörtern (s. Abbildung 5, Abbildung 6 und Abbildung 7). Von 560 Studienteilnehmenden gaben 53 (9,5\%) an, dass Alterssexualität im Laufe ihrer Ausbildung nur negativ thematisiert worden war. 54,1\% $(n=303)$ der Teilnehmenden verneinten dies und weitere 36,4\% $(n=204)$ antworteten mit „Weiß nicht“. Die Items $2.1-2.3$ wurden anhand einer fünfstufigen endpunktverbalisierten Likert-Skala $(1=$ Trifft zu, $5=$ Trifft nicht zu) beantwortet. Knapp ein Drittel der Befragten ( $n=180 ; 31 \%)$ fühlte sich gut auf den Umgang mit verbal und körperlich sexuell übergriffigem Verhalten vorbereitet (s. Abbildung 5). Für 23\% ( $n=134)$ der Befragten traf diese Aussage (eher) nicht zu.

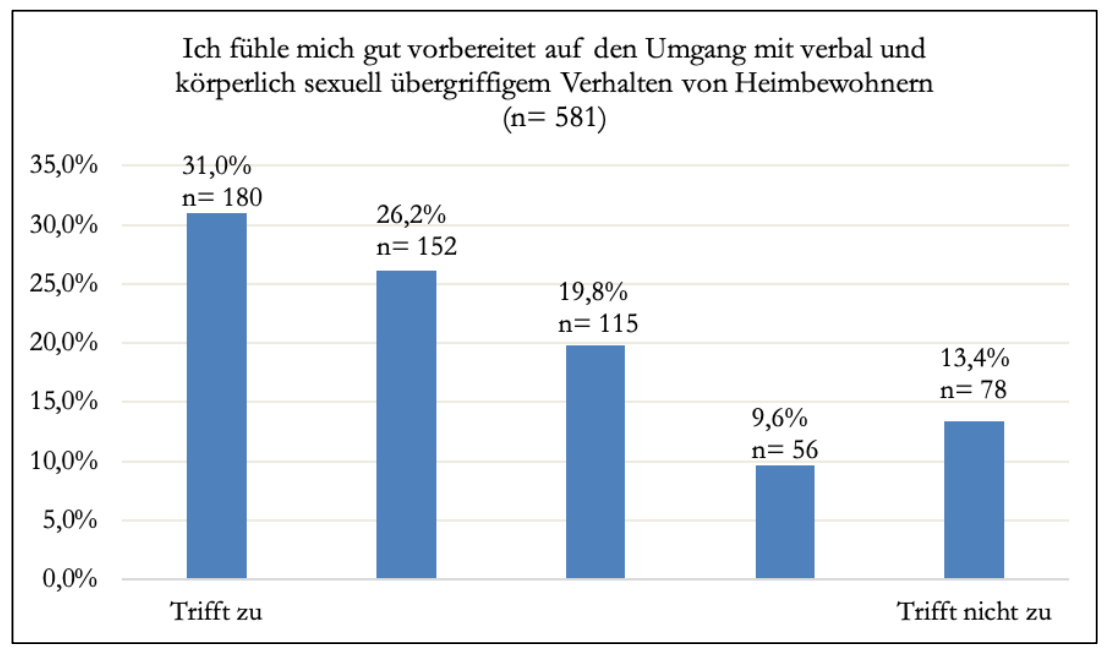

Abbildung 5 Ich fühle mich gut vorbereitet auf den Umgang mit verbal und körperlich sexuell übergriffigem Verhalten von Heimbewohnern $(n=581)$

Die Mehrheit der Studienteilnehmenden ( $\mathrm{n}=261 ; 44,8 \%$ ) gab an, dass das Thema „Umgang mit Alterssexualität“" (eher) nicht in ihrer Ausbildung behandelt wurde. Fast ebenso viele 
Befragten ( $\mathrm{n}=243 ; 41,7 \%$ ) befanden, dass die Aussage „Umgang mit Alterssexualität wurde bei mir in der Ausbildung behandelt" (eher) auf sie zutreffen würde. (s. Abbildung 6).

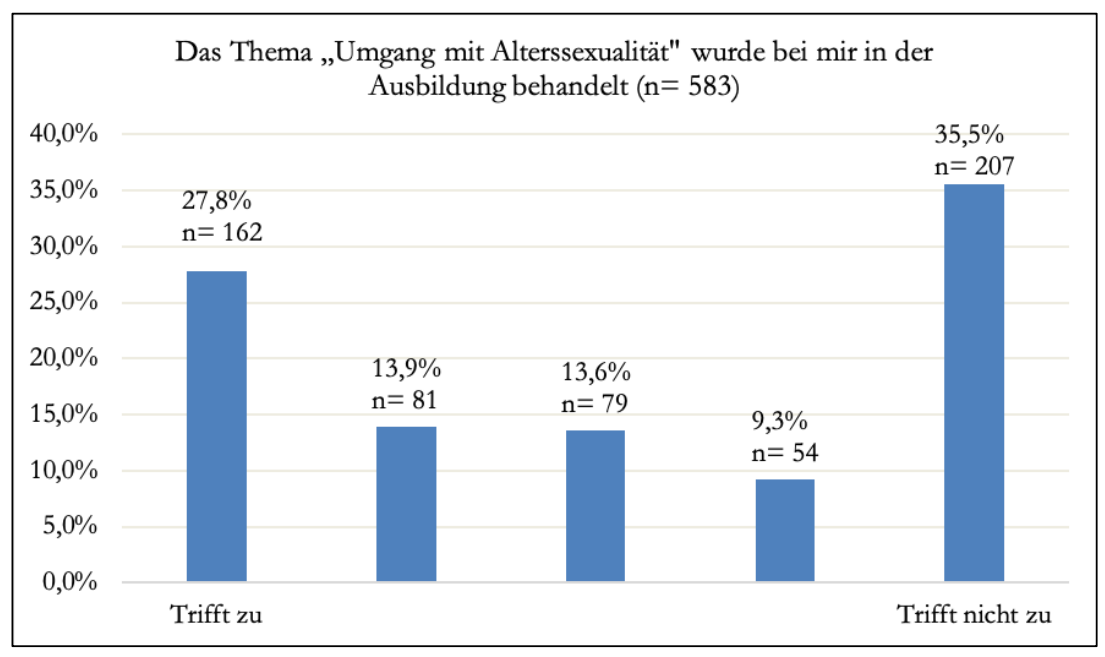

Abbildung 6 Das Thema „Umgang mit Alterssexualität" wurde bei mir in der Ausbildung behandelt $(n=583)$

Ein Großteil ( $\mathrm{n}=390 ; 68,5 \%)$ der befragten Teilnehmenden hielt das Thema „Umgang mit Alterssexualität" für ein für den Arbeitsalltag hilfreiches Ausbildungsfach. Knapp ein Fünftel der Befragten ( $\mathrm{n}=109 ; 19,1 \%$ ) teilte diese Ansicht (eher) nicht (s. Abbildung 7).

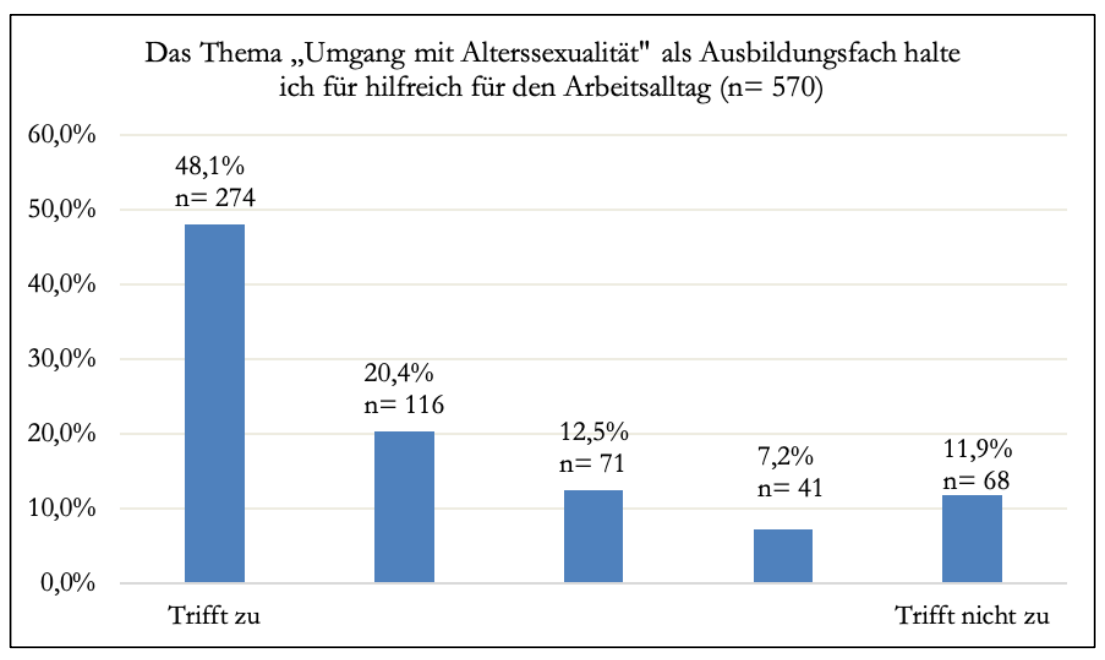

Abbildung 7 Das Thema „Umgang mit Alterssexualität" als Ausbildungsfach halte ich für hilfreich für den Arbeitsalltag $(\mathrm{n}=570)$

Es war ebenfalls Teil der Hauptfrage Q 1, die Ansicht der Lehrenden zum Thema Alterssexualität in der Altenpflegeausbildung zu beleuchten. Der Großteil der befragten Lehrenden gab an, dass das Thema Alterssexualität ein verpflichtender Teil des Curriculums ihrer Schule war $(n=18 ; 78,3 \%)$, gefolgt von der optionalen Behandlung des Themas im 
Unterricht ( $\mathrm{n}=4 ; 17,4 \%)$. Bei 4,3\% ( $\mathrm{n}=1)$ der Befragten war das Thema Alterssexualität kein Bestandteil des Curriculums ( $\left.\mathrm{n}_{\text {gesamt }}=23\right)$.

Die Ergebnisse zu der Frage „Können Sie sich vorstellen, warum die Vermittlung des Themas Alterssexualität von der Schulleitung gewünscht wird?" sind in Tabelle $\mathbf{5}$ dargestellt. Mehrfachnennungen waren erwünscht. Im Bereich „Vermittlung des Themas Alterssexualität" befanden die meisten Lehrenden, dass das Thema wichtig für den Berufsalltag sei $(n=20 ; 66,7 \%)$. Weiterhin würden sich die Schüler wünschen, über das Thema unterrichtet $\mathrm{zu}$ werden $(\mathrm{n}=13 ; 43,4 \%)$. Dem wurde die Frage „Können Sie sich vorstellen, warum die Vermittlung des Themas Alterssexualität von der Schulleitung nicht gewünscht wird?“ gegenübergestellt (s. Tabelle 6). Nicht gewünscht sei die Vermittlung des Themas Alterssexualität vorwiegend aufgrund von Schamgefühlen $(n=6 ; 20 \%)$ sowie aus Unwichtigkeit für den Arbeitsalltag ( $\mathrm{n}=4 ; 13,3 \%)$.

Tabelle 5 Vermittlung des Themas Alterssexualität: Gewünscht $(n=30)$

\begin{tabular}{ll}
\hline Vermittlung des Themas: & Anzahl $n(\%)$ \\
Gewünscht, da es wichtig für den Arbeitsalltag ist & $20(66,7)$ \\
Gewünscht, da es an anderen Schulen unterrichtet wird & $2(6,7)$ \\
Gewünscht, da es einen Wettbewerbsvorteil bietet & $0(0)$ \\
Gewünscht, aus persönlichen Gründen der Schulleitung & $1(3,3)$ \\
Gewünscht, da Schüler darüber unterrichtet werden wollen & $13(43,3)$ \\
Gewünscht aus sonstigen Gründe & $7(23,3)$ \\
\hline
\end{tabular}

Tabelle 6 Vermittlung des Themas Alterssexualität: Nicht gewünscht $(\mathrm{n}=30)$

\begin{tabular}{ll}
\hline Vermittlung des Themas: & Anzahl $n(\%)$ \\
Ungewünscht, weil es unwichtig für den Arbeitsalltag ist & $4(13,3)$ \\
Ungewünscht, weil es zusätzlich kosten verursacht & $3(10)$ \\
Ungewünscht aus Schamgefühlen & $6(20)$ \\
Ungewünscht aus religiösen Gründen & $2(6,7)$ \\
Ungewünscht, weil das Thema an anderen Schulen unterrichtet & $0(0)$ \\
wird & \\
\hline
\end{tabular}

Im Folgenden wird auf die Erweiterung (s. Tabelle 7) und die Aussparung (s. Tabelle 8) des Themas Alterssexualität im Unterricht eingegangen. Die meisten der befragten Lehrenden würden das Thema Alterssexualität aufgrund der subjektiv erachteten Wichtigkeit in den Unterricht einbringen $(n=21 ; 70 \%)$. Auch die Schüler würden sich das Thema Alterssexualität im Unterricht wünschen ( $\mathrm{n}=7 ; 23,3 \%$ ). Dem gegenübergestellt wurde die 
Verringerung/Aussparung des Themas im Unterricht (s. Tabelle 8). Hauptsächlich würde eine Verringerung/Aussparung des Themas aus zeitlichen Gründen erfolgen ( $n=4 ; 13,3 \%$ ). Ebenso viele Lehrende $(n=4 ; 13,3 \%)$ gaben an, dass es den Schülern unangenehm sei, über das Thema unterrichtet zu werden.

Die Mehrzahl der befragten Lehrenden ( $\mathrm{n}=27 ; 96,4 \%$ ) war der Meinung, dass im Unterricht an Altenpflegeschulen auf die Sexualität alter Menschen eingegangen werden sollte $\left(\mathrm{n}_{\text {gesamt }}=\right.$ 28). Von den Befragten trauten sich die meisten zu, das Thema Alterssexualität fachlich zu unterrichten ( $\mathrm{n}=21 ; 75 \%) .93,3 \%(\mathrm{n}=14)$ der Befragten gab an, sich das Unterrichten des Themas nach einer entsprechenden Fortbildung zuzutrauen $\left(\mathrm{n}_{\text {gesamt }}=15\right)$.

Tabelle 7 Erweiterung des Themas Alterssexualität im Unterricht $(n=30)$

\begin{tabular}{ll}
\hline Erweiterung des Themas: & Anzahl $n(\%)$ \\
Ja, weil es vorgeschrieben ist & $1(3,3)$ \\
Ja, weil ich das Thema für wichtig erachte & $21(70)$ \\
Ja, weil es mir Spaß macht, das Thema im Unterricht & $3(10)$ \\
zu bearbeiten & \\
Ja, weil es von den Schülern so gewünscht ist & $7(23,3)$ \\
Ja, weil es von meinem Vorgesetzten so gewünscht ist & $1(3,3)$ \\
Ja, weil es von dem Träger der Schule so gewünscht ist & $0(0)$ \\
\hline
\end{tabular}

Tabelle 8 Verringerung/Aussparung des Themas Alterssexualität im Unterricht $(\mathrm{n}=30)$

\begin{tabular}{ll}
\hline Verringerung/Aussparung des Themas: & Anzahl $n(\%)$ \\
Ja, aus zeitlichen Gründen & $4(13,3)$ \\
Ja, aus finanziellen Gründen & $0(0)$ \\
Ja, weil mir das Thema unangenehm ist & $0(0)$ \\
Ja, weil den Schülern das Thema unangenehm ist & $4(13,3)$ \\
Ja, weil es von meinem Vorgesetzten so gewünscht ist & $0(0)$ \\
Ja, weil es von dem Träger der Schule so gewünscht ist & $1(3,3)$ \\
\hline
\end{tabular}

Im Bereich Weiterbildungsmöglichkeiten/Seminare zum Thema Alterssexualität und sexuelle Gesundheit berichtete die Mehrzahl der befragten Lehrenden ( $n=18 ; 64,3 \%$ ), dass eine derartige Veranstaltung bislang nicht durch die Vorgesetzten angeboten wurde. 10,7 \% $(n=3)$ der befragten Lehrenden hatten bereits an einer Veranstaltung zu diesem Thema teilgenommen. 14,3\% $(n=4)$ der Lehrenden gab an, dass ihre Vorgesetzten derartige Seminare zwar anbieten würden, aber bisher noch keine Teilnahme erfolgt war. Weitere 10,7 \% $(n=3)$ wussten nicht, ob diese Fortbildungsmöglichkeiten bestünden ( $\left.\mathrm{n}_{\text {gesamt }}=28\right) .72,0 \%(\mathrm{n}=18)$ 
der befragten Lehrenden wünschte sich eine Fortbildung zum Thema Alterssexualität $\left(\mathrm{n}_{\text {gesamt }}=25\right)$.

Im Bereich möglicher Wunschthemen einer Fortbildung zu Alterssexualität war das Interesse der Lehrenden ( $\left.\mathrm{n}_{\text {gesamt }}=28\right)$ bezüglich der Unterthemen „Erlernen von didaktischen Fähigkeiten zur Vermittlung des Themas Alterssexualität und sexuelle Gesundheit“ ( $n=20$; 66,7\%) sowie das „Erlernen von Fachwissen“ ( $n=16 ; 53,3 \%)$ am größten. Das geringste Interesse bestand im „Erlernen von Fähigkeiten über das Thema zu reden“ ( $n=12 ; 40 \%)$.

\subsection{Q 2: Welchen Stellenwert hat das Thema Sexualität und sexuelle Gesundheit im Pflegealltag?}

\section{Konzept Sexualassistenz}

Die Items 3.1 - 3.5 befassten sich mit dem Konzept der Sexualassistenz. Vor Beantwortung dieser Items hatten Studienteilnehmende die Möglichkeit, eine kurze Erläuterung zum Thema Sexualassistenz zu lesen. Die Mehrzahl der befragten Studienteilnehmenden ( $\mathrm{n}=301$; 66,9\%) gab an, das Konzept der Sexualassistenz nicht zu kennen. Das Konzept der Sexualassistenz wurde fast ebenso häufig befürwortet ( $n=238 ; 41,5 \%)$, wie sich andere Teilnehmende nicht sicher waren, ob sie es befürworten würden (Weiß nicht: $n=229 ; 40 \%$ ). Ein vergleichsweise geringer Teil der Studienteilnehmenden lehnte eine Befürwortung ab $(\mathrm{n}=106 ; 18,5 \%)$.

Die Umsetzung des Konzeptes der Sexualassistenz kannten 12,7\% ( $\mathrm{n}=73$ ) aus ihrem Berufsalltag in einer Pflegeeinrichtung $\left(\mathrm{n}_{\text {gesamt }}=574\right)$. Nur ein kleiner Teil der Befragten $(\mathrm{n}=$ 51; 8,9\%) gab an, dass das Konzept in ihrer aktuellen Einrichtung umgesetzt wird $\left(\mathrm{n}_{\text {gesamt }}=\right.$ 575). Bei der Mehrzahl der Studienteilnehmenden wurde die Thematik Sexualassistenz nicht im Unterricht angesprochen ( $\mathrm{n}=355 ; 62,5 \%)$. Gut ein Fünftel der Befragten hatte das Thema Sexualassistenz im Unterricht besprochen $(n=126 ; 22,2 \%)$ und weitere 87 Teilnehmende $(15,3 \%)$ wussten nicht, ob eine Thematisierung stattgefunden hatte $\left(\mathrm{n}_{\text {gesamt }}=568\right)$.

\section{Fortbildungsmöglichkeit am Arbeitsplatz}

Die Mehrheit der Studienteilnehmenden ( $\mathrm{n}=367 ; 64,2 \%$ ) gab an, dass Fortbildungen zum Thema Alterssexualität und sexuelle Gesundheit bislang nicht von ihren Vorgesetzten angeboten wurden. 18,0\% $(n=103)$ berichteten, dass eine derartige Veranstaltung zwar angeboten wurde, aber bisher noch keine Teilnahme erfolgt war. Ein geringer Teil der Befragten ( $\mathrm{n}=30 ; 5,2 \%)$ hatte bereits an einer Fortbildung zum Thema Alterssexualität und sexuelle Gesundheit teilgenommen. Die restlichen Teilnehmenden ( $\mathrm{n}=72 ; 12 \%)$ wussten nicht, ob ein derartiges Fortbildungsprogramm besteht. Eine Vielzahl der Teilnehmenden 
( $n=396 ; 71,9 \%)$ wünscht sich eine Fortbildung zum Thema Alterssexualität und sexuelle Gesundheit.

Im Bereich möglicher Wunschthemen einer Fortbildung zu Alterssexualität und sexueller Gesundheit war das Interesse der Teilnehmenden $\left(\mathrm{n}_{\text {gesamt }}=598\right)$ bezüglich des Unterthemas „Umgang mit sexuell aktiven Heimbewohnern“ ( $\mathrm{n}=350 ; 58,5 \%)$ am größten. Ähnlich groß war das Interesse bezüglich der Themen „Erlernen von Fähigkeiten über sexuelle Vorkommnisse in der Bewohnerversorgung zu reden“ ( $n=224 ; 37,5 \%)$ und dem „Erlernen von Fachwissen“ ( $n=217 ; 36,3 \%$ ). Nur ein kleiner Teil der Befragten gab an, eine solche Fortbildung nicht besuchen zu wollen ( $\mathrm{n}=55 ; 9,2 \%)$.

42,0\% ( $n=226)$ der Befragten gaben an, sich eine offizielle Anlaufstelle zu wünschen, um über sexuelle Vorkommnisse in der Bewohnerversorgung zu reden ( $\left.\mathrm{n}_{\text {gesamt }}=538\right) .24,7 \%$ ( $\mathrm{n}=$ 133) der Befragten wünschten und hatten eine solche Anlaufstelle und weitere 33,3\% (n= 179) gaben an, keine solche Anlaufstelle zu benötigen. In der untenstehenden Tabelle 9 sind die Ergebnisse der möglichen Anlaufstellen für Vorkommnisse und Probleme in der Bewohnerversorgung aufgelistet (Mehrfachnennungen waren möglich). Etwas über ein Fünftel $(21,4 \%)$ der Teilnehmenden berichtet, keine offizielle Anlaufstelle zu haben.

Tabelle 9 Am Arbeitsplatz habe ich eine offizielle Anlaufstelle, um über sexuelle Vorkommnisse/Probleme in der Bewohnerversorgung zu reden $(\mathrm{n}=598)$

\begin{tabular}{ll}
\hline Anlaufstelle: & Anzahl $n(\%)$ \\
Betreuer/Supervisor & $52(8,7)$ \\
Betriebsrat & $38(6,4)$ \\
Pflegedienstleitung & $195(32,6)$ \\
Kollegen & $234(39,1)$ \\
Vorgesetzte & $114(19,1)$ \\
Keine & $128(21,4)$ \\
Weiß nicht & $107(17,9)$ \\
\hline
\end{tabular}

\section{Situationen in meiner Einrichtung/meinem Wohnbereich}

Der fünfte Teil des Fragebogens „Situation in meiner Einrichtung/meinem Wohnbereich“ befasste sich mit verschiedenen Aspekten zum Thema Alterssexualität und sexuelle Gesundheit im Pflegealltag. Im Folgenden werden Ergebnisse zu sexuellen Übergriffen und Paarbildung dargestellt.

Etwas über die Hälfte $(\mathrm{n}=261 ; 54,2 \%$ ) der Studienteilnehmenden gab an, zum Erhebungszeitpunkt keine sexuelle Aktivität unter Heimbewohnenden erlebt zu haben. 45,8\% ( $\mathrm{n}=259)$ der Studienteilnehmenden hatte sexuelle Aktivität unter Heimbewohnenden 
erlebt. Studienteilnehmende wurden gefragt, wie häufig sie Paarbildungen unter Heimbewohnenden sowie unter Heimbewohnenden und Externen erlebt hatten. Bewusst wurden im Fragebogen die Antwortoptionen „Nie“, „Gelegentlich“ und „Regelmäßig“ gewählt, um so die subjektiv wahrgenommene Häufigkeit der Studienteilnehmenden zu diesem Thema zu eruieren (s. Tabelle 10). So schätzten die Studienteilnehmenden, dass es am häufigsten zu einer ,gelegentlichen“ Paarbildung kommen würde (Unter Heimbewohnenden $n=405 ; 71,6 \%$ und unter Heimbewohnenden und Externen $n=279$; $50,2 \%)$.

Tabelle 10 Paarbildung in meiner Einrichtung/meinem Wohnbereich

\begin{tabular}{llll}
\hline Paarbildung: & Nie $n(\%)$ & Gelegentlich $n(\%)$ & Regelmäßig $n(\%)$ \\
$\begin{array}{l}\text { Unter Heimbewohnenden } \\
(\mathrm{n}=566)\end{array}$ & $126(22,3)$ & $405(71,6)$ & $35(6,2)$ \\
$\begin{array}{l}\text { Unter Heimbewohnenden } \\
\text { und Externen }(\mathrm{n}=556)\end{array}$ & $244(43,9)$ & $279(50,2)$ & $33(5,9)$ \\
\hline
\end{tabular}

Die Items 5.6 bis 5.11 befassten sich mit den Themen körperliche und verbale sexuelle Übergriffe. Diese sollten jeweils in Bezug zu verschiedenen Konstellationen gesetzt werden (bspw. sexuelle Übergriffe unter Heimbewohnenden oder zwischen Pflegenden und Heimbewohnenden). Die Beantwortung erfolgte anhand von Zeitangaben (Täglich, Wöchentlich, Monatlich, Seltener, Nie) und wurden der persönlichen Erfahrung nach beantwortet. Die Ergebnisse wurden in den untenstehenden Abbildungen zusammengefasst (s. Abbildung 8, Abbildung 9 und Abbildung 10). Insgesamt hatten 23,9\% ( $\mathrm{n}=136)$ der Studienteilnehmenden mindestens monatlich verbale sexuelle Übergriffe durch Gepflegte miterlebt. 21,9\% $(n=124)$ der Befragten berichteten über mindestens monatlich erlebte körperliche sexuelle Übergriffe durch Heimbewohnende. Die Anzahl der durch Heimbewohnende auf Pflegende stattgefundenen sexuellen Übergriffe machten den Großteil der Übergriffe aus. Dennoch zeigt sich, dass es sowohl von Seiten der Heimbewohnenden als auch von Pflegenden ausgehend zu sexueller Gewaltausübung kommt. 


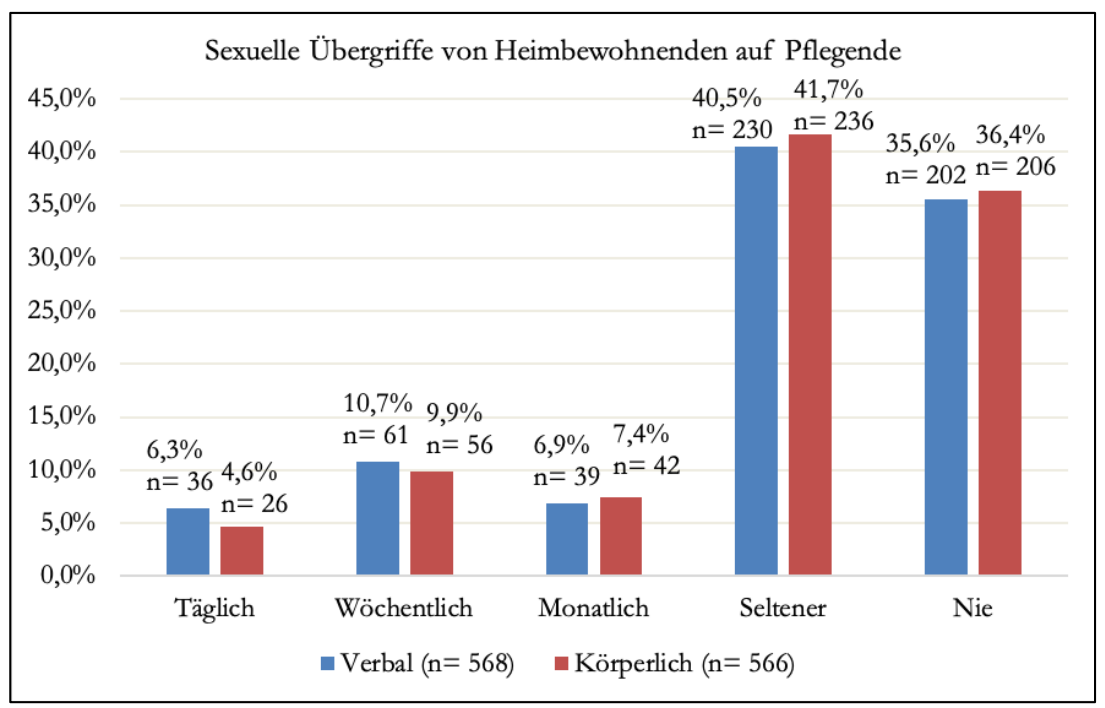

Abbildung 8 Sexuelle Übergriffe von Heimbewohnenden auf Pflegende

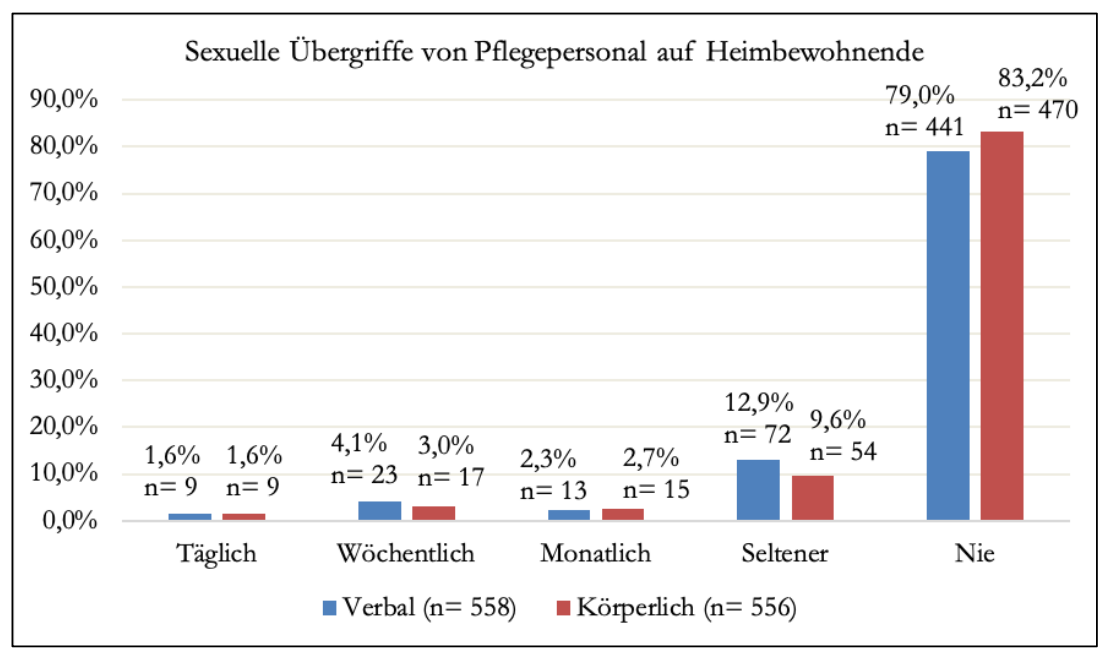

Abbildung 9 Sexuelle Übergriffe von Pflegepersonal auf Heimbewohnende

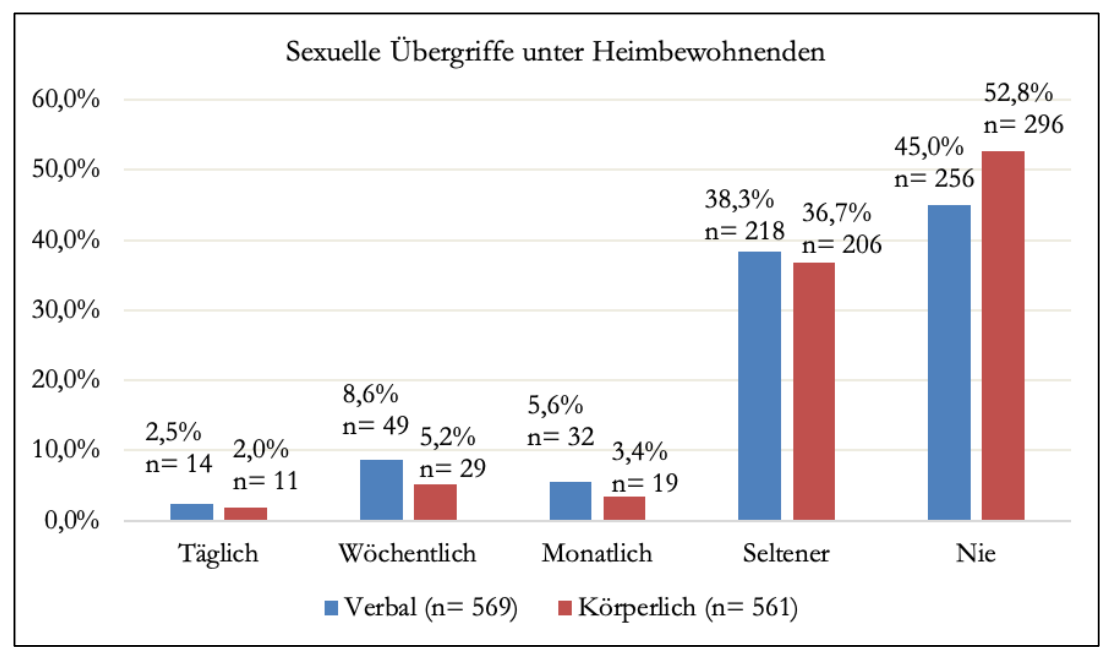

Abbildung 10 Sexuelle Übergriffe unter Heimbewohnenden 
Insgesamt hatten von 342 Studienteilnehmenden 128 (37,4\%) der Befragten Situationen wie Selbstbefriedigung, Auffinden von erotischen Zeitschriften sowie sexuelle Belästigung mehrheitlich bei Männern beobachtet. 35 Teilnehmende (10,2\%) gaben an, diese Situationen bei Männern und Frauen gleichermaßen beobachtet zu haben.

\section{Situation in meinem Berufsalltag}

Im Teil 6 des Fragebogens sollten Teilnehmende verschiedene Situationen in ihrem Berufsalltag ihrer Häufigkeit nach benennen. Die Items 6.1 bis 6.13 befassten sich mit dem Auffinden und der Bereitstellung von sexuellen Hilfsmitteln. Die Häufigkeiten des Auffindens sexueller Hilfsmittel (Items 6.1 - 6.7) wurden in Tabelle 11 aufgezeigt. Im Fragebogen wurden die Häufigkeiten zeitlich skaliert: Täglich, Wöchentlich, Monatlich, Seltener und Nie. Die Optionen Täglich, Wöchentlich und Monatlich wurden im Folgenden als zeitliche Angabe „Häufiger“ zusammengefasst (s. Tabelle 11). Dem gegenübergestellt wurden die zeitlichen Angaben Seltener und Nie. Seltener kam es zum Auffinden insbesondere von Zeitschriften $(n=104 ; 29,5 \%)$ und Videos/Filmen mit pornographischem Inhalt $(n=83 ; 24,3 \%)$. Zu den häufiger aufgefundenen Hilfsmitteln gehörten ebenfalls Zeitschriften $(n=55 ; 15,65 \%)$ und Videos/Filme mit pornographischem Inhalt $(n=47$; $13,7 \%)$.

Tabelle 11 Häufigkeit: Auffinden sexueller Hilfsmittel

\begin{tabular}{lllll}
\hline Sexuelle Hilfsmittel: & $\begin{array}{l}\text { Häufiger } n \\
(\%)\end{array}$ & Seltener $n(\%)$ & $\begin{array}{l}\text { Nie } n \\
(\%)\end{array}$ & Anzahl gesamt \\
Dildos & $16(4,6)$ & $34(9,7)$ & $300(85,7)$ & 350 \\
Gleitgel & $15(4,3)$ & $28(8)$ & $306(87,7)$ & 349 \\
Penisring & $9(2,6)$ & $16(4,5)$ & $327(92)$ & 352 \\
Potenzmittel & $21(5,9)$ & $43(12,1)$ & $292(82)$ & 356 \\
Vibrator & $19(5,4)$ & $30(8,6)$ & $301(86)$ & 350 \\
Videos/Film & $47(13,7)$ & $83(24,3)$ & $212(62)$ & 342 \\
Zeitschriften & $55(15,6)$ & $104(29,5)$ & $194(55)$ & 353 \\
\hline
\end{tabular}

Darüber hinaus wurden Teilnehmende gefragt, welche sexuellen Hilfsmittel von ihrer Einrichtung zur Verfügung gestellt wurden und ob sie die Bereitstellung sexueller Hilfsmittel in ihrer Einrichtung für sinnvoll erachten würden (s. Tabelle 12, Mehrfachnennungen waren möglich). Die konkrete Bereitstellung sexueller Hilfsmittel durch die Einrichtung wurde als Reale Bereitstellung aufgeführt. Die Ergebnisse zur Frage nach der sinnvollen Bereitstellung sexueller Hilfsmittel wurden als Sinnvolle Bereitstellung bezeichnet. Die Mehrheit der Studienteilnehmenden $(\mathrm{n}=91 ; 66,1 \%)$ gab an, dass keine sexuellen Hilfsmittel von ihrer 
Einrichtung zur Verfügung gestellt wurden. Videos und Filme mit pornographischem Inhalt waren die am häufigsten von einer Einrichtung zur Verfügung gestellten sexuellen Hilfsmittel $(\mathrm{n}=12 ; 3,2 \%)$. Weiterhin war sich die Mehrheit der Befragten $(\mathrm{n}=137 ; 36,2 \%)$ nicht sicher, ob eine Bereitstellung sexueller Hilfsmittel in ihrer Einrichtung sinnvoll wäre. $31 \%$ ( $n=117$ ) der Befragten erachteten die Bereitstellung sexueller Hilfsmittel als nicht sinnvoll.

Tabelle 12 Reale und sinnvolle Bereitstellung sexueller Hilfsmittel in Einrichtungen $(n=378)$

\begin{tabular}{lll}
\hline Sexuelle Hilfsmittel: & Reale Bereitstellung $n(\%)$ & Sinnvolle Bereitstellung $n(\%)$ \\
Dildos & $1(0,3)$ & $38(10,1)$ \\
Gleitgel & $7(1,9)$ & $30(7,9)$ \\
Penisring & $2(0,5)$ & $24(6,3)$ \\
Potenzmittel & $0(0)$ & $33(8,7)$ \\
Vibrator & $6(1,0)$ & $40(10,6)$ \\
Videos/Film & $12(3,2)$ & $59(15,6)$ \\
Zeitschriften & $4(1,1)$ & $71(18,8)$ \\
Keine & $91(66,1)$ & $117(31)$ \\
Weiß nicht & $11(24,1)$ & $137(36,2)$ \\
\hline
\end{tabular}

Studienteilnehmende hatten außerdem die Gelegenheit, anhand zwei offener Fragen (Items 6.10, 6.12) weitere sexuelle Hilfsmittel zu nennen, die von ihrer Einrichtung zur Verfügung gestellt wurden. Dabei wurden Kondome $(n=1)$, Prostituierte $(n=2)$, Liebesschaukeln $(n=$ 2) und Sexualassistenten ( $\mathrm{n}=1)$ genannt. Ebenfalls wurden Alltagsgegenstände aufgeführt, bspw. Löffel und Schnabelbecher $(n=1)$ oder Fernbedienungen $(n=1)$. Des Weiteren konnten Teilnehmende sexuelle Hilfsmittel nennen, deren Bereitstellung in Einrichtungen erfolgen sollte: Prostituierte $(n=4)$, Dildos $(n=4)$, Potenzmittel $(n=2)$, Kondome $(n=1)$ sowie einen Raum zum Zurückziehen ( $\mathrm{n}=1)$.

378 der Studienteilnehmenden beantworteten, inwieweit sie die Bereitstellung sexueller Hilfsmittel in Einrichtungen als sinnvoll erachteten (s. Tabelle 13; Mehrfachnennungen waren möglich). Eine Vielzahl der Teilnehmenden ( $n=140 ; 37 \%)$ befand die Bereitstellung sinnvoll, da ältere Menschen sexuell aktiv sein wollen. Ähnlich viele Teilnehmende ( $\mathrm{n}=139$; 36,8\%) wussten nicht, ob die Bereitstellung sexueller Hilfsmittel in einer Einrichtung sinnvoll wäre. 
Tabelle 13 Bereitstellung sexueller Hilfsmittel durch die Pflegeeinrichtung ( $\mathrm{n}=378$ )

\begin{tabular}{ll}
\hline Die Bereitstellung von sexuellen Hilfsmitteln ist: & Zustimmung $n(\%)$ \\
Sinnvoll, weil Bewohner selber keine beschaffen können & $87(23)$ \\
Sinnvoll, wegen körperlicher Einschränkungen bei sexuellen & $60(15,9)$ \\
Handlungen im Alter & $140(37)$ \\
Sinnvoll, da ältere Menschen sexuell aktiv sein wollen & $35(9,3)$ \\
Sinnvoll, aber zu teuer & \\
& $21(5,6)$ \\
Nicht sinnvoll, da ältere Menschen nicht sexuell aktiv sind & $12(3,2)$ \\
Nicht sinnvoll, da trotz Hilfsmitteln keine sexuelle Aktivität & \\
mehr möglich ist & $16(4,2)$ \\
Nicht sinnvoll, da Sexualität bei Älteren abstoßend ist & $139(36,8)$ \\
Weiß nicht &
\end{tabular}

Studienteilnehmende nannten Möglichkeiten, die Bewohnende für das ungestörte Ausleben ihrer Sexualität hatten ( $\mathrm{n}_{\text {gesamt }}=378$; Mehrfachnennungen waren möglich). Die am häufigsten genannte Option war das Abschließen von Zimmern ( $n=155 ; 41 \%)$, gefolgt von der Möglichkeit, dass Paare in Doppelzimmern wohnen konnten ( $\mathrm{n}=145 ; 38,4 \%$ ). Weiterhin gaben 7,1\% $(n=27)$ der Teilnehmenden an, dass es „Bitte-nicht-stören-Schilder“ für die Türen der Bewohnenden gab. 2,4\% $(n=9)$ der Befragten berichteten über spezielle Räumlichkeiten und Rückzugsorte für Paare. 15,1\% ( $n=57)$ der Befragten sagten, dass es keine Möglichkeiten für Heimbewohnende gäbe, ungestört ihre Sexualität auszuleben. 23,5\% $(\mathrm{n}=89)$ antworteten mit ,Weiß nicht“.

\section{Meinungsbild}

Im siebten Teil des Fragebogens Meinungsbild wurden Reaktionen zum Thema sexuelle Aktivität und Partnerschaften unter Heimbewohnern erfragt. Von 570 Studienteilnehmenden gaben $246(43,2 \%)$ an, dass sexuelle Aktivität unter Heimbewohnern vom Pflegepersonal toleriert werde. 26,7\% $(n=151)$ der Befragten meinten, dass sexuelle Aktivität unter Heimbewohnenden von anderen Heimbewohnenden toleriert werde und weitere 15,0\% ( $\mathrm{n}=85)$ sagten, dass Angehörige sexuelle Aktivität unter Heimbewohnenden tolerieren würden $\left(\mathrm{n}_{\text {gesamt }}=565\right)$. Die subjektiv am häufigsten erlebten Reaktionen auf sexuelle Aktivität unter Heimbewohnenden sind in der untenstehenden Abbildung 11 dargestellt. „Ekel“ (33,1\%) und „Es lächerlich machen“ (31,4\%) waren dabei die häufigsten Reaktionen des Pflegepersonals. 


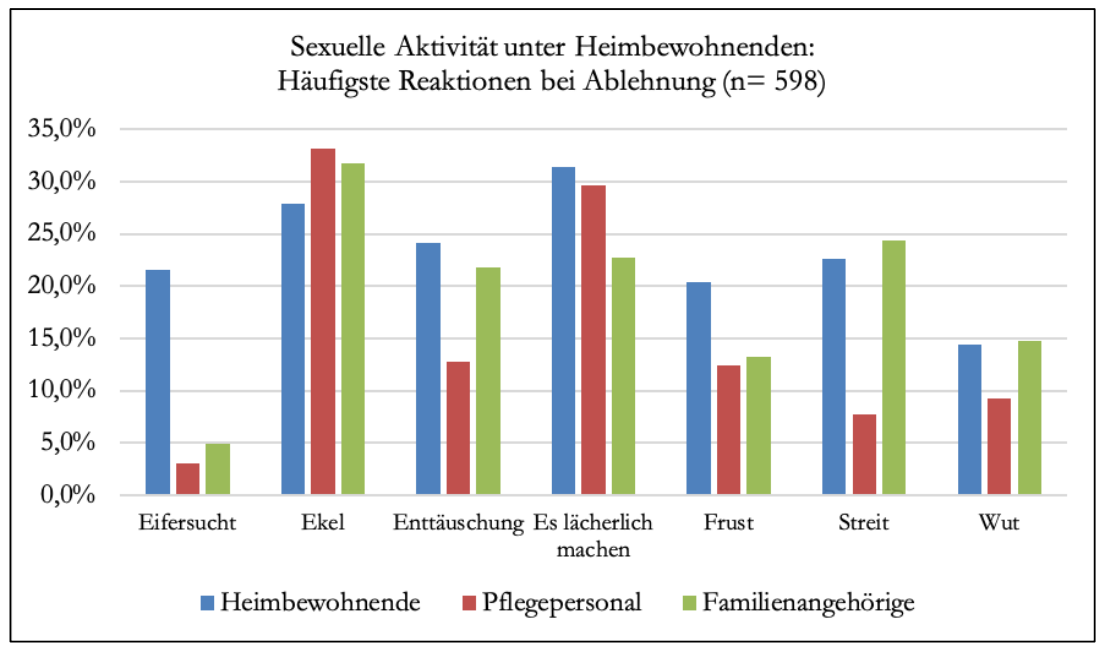

Abbildung 11 Sexuelle Aktivität unter Heimbewohnenden: Häufigste Reaktionen bei Ablehnung $(\mathrm{n}=598)$

Des Weiteren wurde die Toleranz gegenüber der Neubildung von Partnerschaften unter Heimbewohnenden erfragt. Von 559 Befragten gab fast die Hälfte an ( $\mathrm{n}=297 ;$ 49,9\%), dass andere Heimbewohnenden die Neubildung von Partnerschaften nicht tolerieren würden. $58,3 \% \quad(\mathrm{n}=327)$ der Studienteilnehmenden schätzten, dass die Neubildung von Partnerschaften unter Heimbewohnenden vom Pflegepersonal toleriert werde ( $\left.\mathrm{n}_{\text {gesamt }}=561\right)$. 26,4\% ( $n=146)$ der Befragten sagten, dass eine Neubildung von Partnerschaften von Familienangehörigen toleriert werde $\left(\mathrm{n}_{\text {gesamt }}=554\right)$. Die subjektiv am häufigsten erlebten Reaktionen auf die Neubildung von Partnerschaften unter Heimbewohnenden sind in der untenstehenden Abbildung 12 aufgeführt. Am häufigsten wurden neue Partnerschaften unter Heimbewohnenden sowohl vom Pflegepersonal (27,6\%) als auch von anderen Heimbewohnenden $(24,1 \%)$ lächerlich gemacht. Angehörige reagierten häufig mit Enttäuschung auf die Neubildung von Partnerschaften (23,4\%).

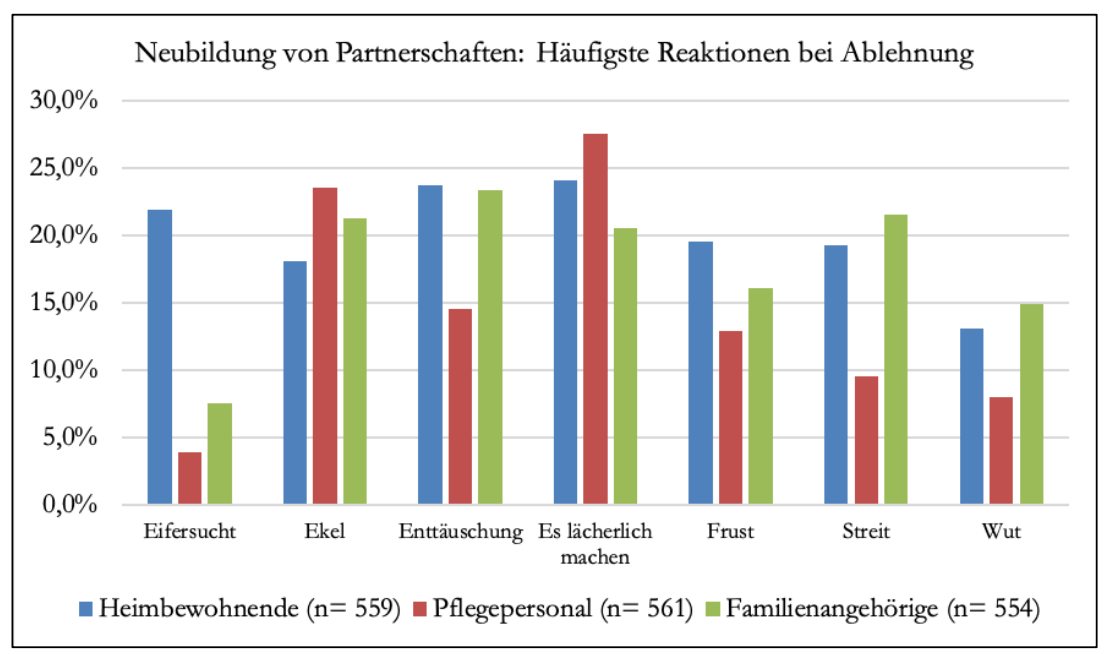

Abbildung 12 Neubildung von Partnerschaften: Häufigste Reaktionen bei Ablehnung 
Meinungsbild: Welche Bedeutung haben sexuell übertragbare Erkrankungen und die ärztliche Versorgung in der Altenpflege?

$37,5 \%(n=212)$ der Studienteilnehmenden gaben an, dass von Seiten der Pflege auf Vorsorgeuntersuchungen durch Gynäkologen und Urologen geachtet wird $\left(\mathrm{n}_{\text {gesamt }}=565\right)$. Die Mehrheit der Befragten ( $\mathrm{n}=380 ; 68,1 \%)$ erachteten diese Art von Arztbesuch als sinnvoll $\left(\mathrm{n}_{\text {gesamt }}=558\right) .598$ Studienteilnehmende beantworteten Fragen zu sexuell übertragbaren Erkrankungen (Sexually transmitted diseases= STDs) in ihrem Berufsalltag (s. Tabelle 14). Die am häufigsten erlebten Erkrankungen waren Pilzinfektionen ( $n=338 ; 56,6 \%$ ), gefolgt von Hepatitis B und C $(n=215 ; 36 \%)$ und HIV ( $n=135 ; 22,6 \%)$.

Tabelle 14 Mit welchen der folgenden sexuell übertragbaren Erkrankungen hatten Sie in Ihrem Berufsalltag in der Altenpflege schon zu tun? $(\mathrm{n}=598)$

\begin{tabular}{ll}
\hline Sexuell übertragbare Erkrankungen: & Anzahl $n(\%)$ \\
Chlamydien & $87(14,5)$ \\
Gonorrhoe (Tripper) & $44(7,4)$ \\
Hepatitis B und C & $215(36)$ \\
Herpes genitalis & $98(16,4)$ \\
Human Immunodeficiency Virus (HIV) & $135(22,6)$ \\
Humanes Papilloma Virus (HPV) & $56(9,4)$ \\
Pilzinfektionen & $338(56,6)$ \\
Treponema pallidum (Syphilis) & $34(5,7)$ \\
\hline
\end{tabular}

Zudem sollten die Befragten angeben, wie viel Altenpflegende $(n=557)$ und Bewohnende ( $\mathrm{n}=548$ ) zum Thema STDs wussten (s. Abbildung 13). Generell schienen die Studienteilnehmenden Altenpflegende für informierter zu halten als Heimbewohnende. Die Mehrzahl der Teilnehmenden $(n=399 ; 70,1 \%)$ meinte, es wäre wichtig, sexuell aktive Heimbewohnende über sexuell übertragbare Erkrankungen aufzuklären. 11,2\% ( $n=64)$ der Befragten halten dies nicht für notwendig $\left(\mathrm{n}_{\text {gesamt }}=569\right)$. 


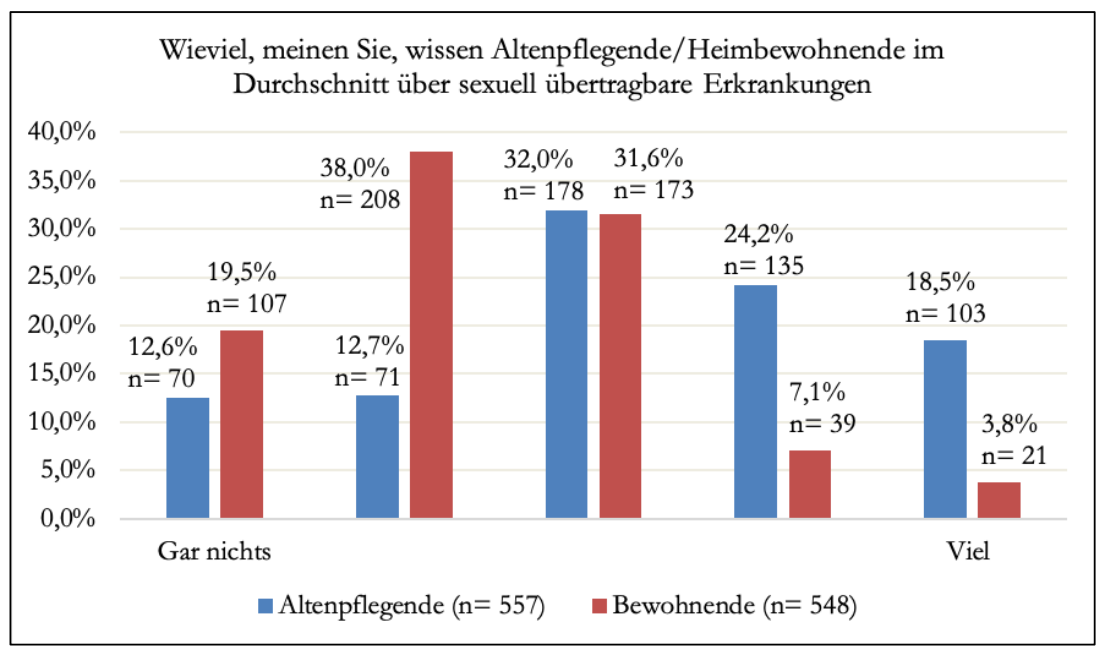

Abbildung 13 Wieviel, meinen Sie, wissen Altenpflegende/Heimbewohnende im Durchschnitt über sexuell übertragbare Erkrankungen?

\subsection{Q 3: Was ist der aktuelle Wissensstand in der Altenpflege zum Thema Sexualität und sexuelle Gesundheit?}

Im Folgenden wird anhand der Items 7.20 bis 7.24 auf den Wissensstand der Teilnehmenden zum Thema Sexualität eingegangen. Die meisten Studienteilnehmenden beantworteten die Frage, ob der Mann aus biologischen Gründen sexuell aktiver sei als die Frau falsch ( $n=204$; 37,4\%). Die richtige Antwort „Nein“ kannten 147 Studienteilnehmende (27\%). Die restlichen Befragten antworteten mit „Weiß nicht“ ( $n=77 ; 35,6 \%)$. Im Gegensatz dazu wusste die Mehrzahl der Studienteilnehmenden ( $\mathrm{n}=362 ; 70 \%$ ), dass die Orgasmusfähigkeit von Frauen auch nach den Wechseljahren erhalten bleibt. Ein kleiner Teil der Befragten beantwortete diese Frage falsch $(n=8 ; 1,5 \%)$. Dagegen betrug der Anteil der Studienteilnehmenden, die es nicht wussten, fast ein Drittel ( $\mathrm{n}=147 ; 28,4 \%)$.

36,7\% $(n=196)$ der Teilnehmenden bejahten, dass Frauen aus biologischen Gründen sexuelle Abstinenz besser ertragen würden als Männer. 14,8\% $(\mathrm{n}=79)$ verneinten dies. Die Mehrzahl der Studienteilnehmenden ( $\mathrm{n}=259 ; 48,5 \%)$ antwortete mit „Weiß nicht“ ( $\mathrm{n}_{\text {gesamt }}=$ 534). Weiterhin wusste die Mehrzahl der Studienteilnehmenden ( $\mathrm{n}=349 ; 63,3 \%$ ), dass häufiges Masturbieren nicht psychisch schädlich ist. 26,9\% $(n=148)$ beantwortete die Frage mit „Weiß nicht“" und 9,8\% $(n=54)$ der Befragten beantwortete die Frage falsch. Mit einer Mehrheit von 77,8\% ( $\mathrm{n}=432)$ wussten die Studienteilnehmenden, dass Homosexualität keine Krankheit ist. 22,2\% der Teilnehmenden lagen mit ihren Antworten zu der Frage „Ist Homosexualität eine Krankheit?“ falsch (Ja: $n=46 ; 8,3 \%$. Weiß nicht: $n=77 ; 13,9 \%$ ).

Insgesamt wurden die Items zu Wissen um Sexualität oft falsch oder mit „Weiß nicht“ beantwortet. 


\subsection{Q 4: Wie wird die eigene Sexualität, die Sexualität älterer Menschen und die Sexualität eigener Angehöriger wahrgenommen?}

Die Mehrzahl der Studienteilnehmenden $(n=423 ; 75,5 \%)$ gab an, älteren Menschen das aktive Ausleben von Sexualität zu gönnen. 62 (11,1\%) Teilnehmende verneinten dies. Der Rest ( $\mathrm{n}=75 ; 13,4 \%)$ beantwortete diese Frage mit „Weiß nicht“ ( $\left.\mathrm{n}_{\text {gesamt }}=560\right)$. Die Antworten auf die Frage „Gönnen Sie es alten Menschen, ihre Sexualität aktiv auszuleben?“ in Bezug auf das Alter der Studienteilnehmenden wurden in Tabelle 15 aufgeführt. Hier zeigte sich in der Befürwortung einer aktiven Sexualität im Alter der deutlichste Unterschied zwischen der Gruppe der 35- bis 45-Jährigen $(n=63 ; 90 \%)$ und der Gruppe der $>60$-Jährigen $(n=14$; 53,8\%). Die Gruppe der > 60-Jährigen hatte - prozentual gesehen - den größten Anteil der ablehnenden Teilnehmenden $(\mathrm{n}=6 ; 23,1 \%)$, gefolgt von der Altersgruppe der 50- bis 60 Jährigen $(n=3 ; 16,7 \%)$.

Tabelle 15 Gönnen Sie es alten Menschen, ihre Sexualität aktiv auszuleben? ( $\mathrm{n}=518$ )

\begin{tabular}{lllll}
\hline Altersgruppe [Jahre] & Ja $n(\%)$ & Nein $n(\%)$ & Weiß nicht $n(\%)$ & $\begin{array}{l}\text { Altersgruppe gesamt } \\
(\mathrm{n}=518)\end{array}$ \\
$<25$ & $156(76,1)$ & $22(10,7)$ & $27(13,2)$ & 205 \\
$25-35$ & $100(74,1)$ & $19(14,1)$ & $16(11,9)$ & 135 \\
$35-45$ & $63(90)$ & $4(5,7)$ & $3(4,3)$ & 70 \\
$45-55$ & $55(85,9)$ & $2(3,1)$ & $7(10,9)$ & 64 \\
$55-60$ & $12(66,7)$ & $3(16,7)$ & $3(16,7)$ & 18 \\
$>60$ & $14(53,8)$ & $6(23,1)$ & $6(23,1)$ & 26 \\
\hline
\end{tabular}

Die Mehrzahl der Studienteilnehmenden ( $\mathrm{n}=385 ; 70,8 \%$ ) sah es als positiv an, wenn ältere Menschen ein (erfülltes) Sexualleben haben. 10,4\% $(n=55)$ der Befragten beantworteten mit „Nein“ und weitere 19,1\% ( $\mathrm{n}=104)$ mit „Weiß nicht“ ( $\left.\mathrm{n}_{\text {gesamt }}=544\right)$.

Eine Vielzahl der Studienteilnehmenden ( $\mathrm{n}=418 ; 76,4 \%$ ) sah das Ausleben von Sexualität im Alter als etwas Natürliches an. 15,9\% $(n=87)$ der Befragten wussten nicht, ob dies etwas Natürliches sei und 7,7\% $(n=87)$ verneinten es. Auch hier zeigte sich die Altersgruppe der $>60-J a ̈ h r i g e n$ am restriktivsten in der Befürwortung einer aktiven Sexualität im Alter (Ja: $n=$ 9; 36\%, s. Tabelle 16). 
Tabelle 16 Das aktive Ausleben von Sexualität im Alter ist etwas Natürliches ( $\mathrm{n}=509$ )

\begin{tabular}{lllll}
\hline Altersgruppe [Jahre] & Ja $n(\%)$ & Nein $n(\%)$ & Weiß nicht $n(\%)$ & $\begin{array}{l}\text { Altersgruppe gesamt } \\
(\mathrm{n}=509)\end{array}$ \\
$<25$ & $145(71,4)$ & $14(6,9)$ & $44(21,7)$ & 203 \\
$25-35$ & $110(83,3)$ & $6(4,5)$ & $16(12,1)$ & 132 \\
$35-45$ & $65(92,9)$ & $2(2,9)$ & $3(4,3)$ & 70 \\
$45-55$ & $59(90,8)$ & $1(1,5)$ & $5(7,7)$ & 65 \\
$55-60$ & $9(64,3)$ & $1(7,1)$ & $4(28,6)$ & 14 \\
$>60$ & $9(36)$ & $9(36)$ & $7(28)$ & 25 \\
\hline
\end{tabular}

In Abbildung 14 wurde die Akzeptanz von sexuell aktiven Familienmitgliedern (Eltern $n=$ 544 und Großeltern n= 534) vergleichend gegenübergestellt. Hier zeigte sich, dass über die Hälfte der Teilnehmenden ihre Eltern $(n=313 ; 57,5 \%)$ und Großeltern $(n=268 ; 50,2 \%)$ als sexuell aktiv akzeptierten.

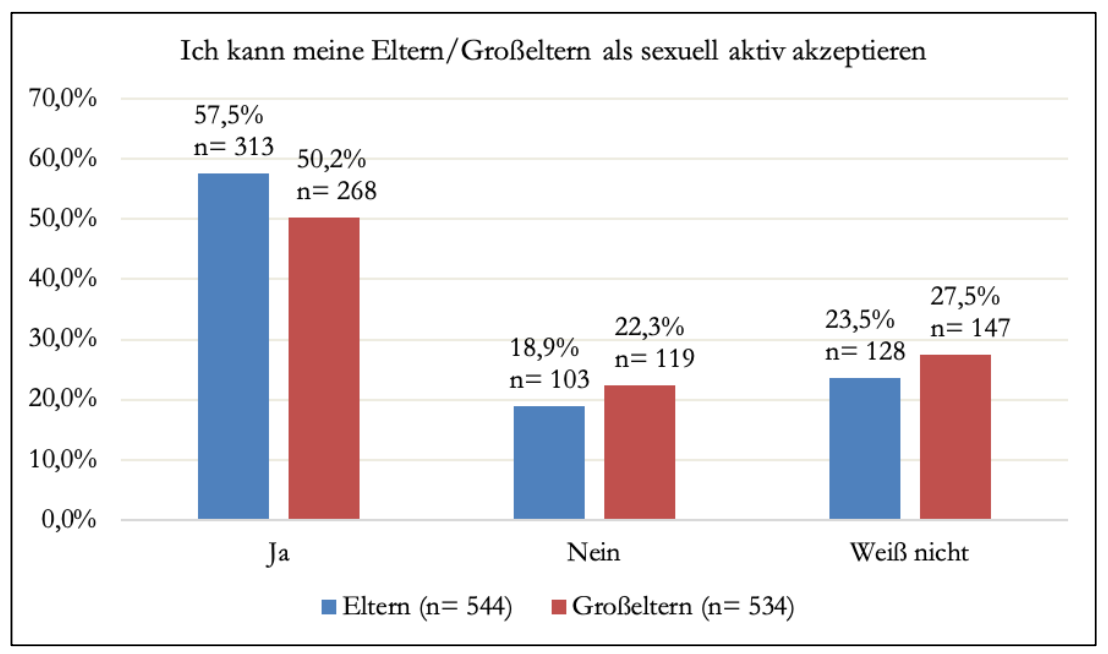

Abbildung 14 Ich kann meine Eltern/Großeltern als sexuell aktiv akzeptieren

Die Items 8.1 sowie 8.7 bis 8.13 dienten der Reflexion des eigenen Sexualverhaltens. Anhand einer fünfstufigen Likert-Skala $(1=$ Trifft $\mathrm{zu}, 5=$ Trifft nicht $\mathrm{zu})$ trafen die Studienteilnehmenden Aussagen zu ihrer eigenen Sexualität und ihrem sexuellen Verhalten. Die deskriptive Darstellung erfolgte in Abbildung 15 und Abbildung 16. Die Mehrzahl der Studienteilnehmenden $(n=287 ; 60,4 \%)$ beantwortete die Fragen basierend auf einer aktuellen Beziehung. 27,4\% $(n=130)$ der Befragten antworteten auf der Basis einer vergangenen Beziehung und 12,2\% ( $\mathrm{n}=58)$ anhand einer hypothetischen Beziehung ( $\mathrm{n}_{\text {gesamt }}=$ 475). Insgesamt zeigten sich die Teilnehmenden gegenüber ihrer eigenen Sexualität eher aufgeschlossen. Die Mehrzahl der Befragten war sich ihrer sexuellen Gefühle sehr bewusst $(68,2 \%)$ und befand auch, dass sie für ihre Sexualität zum Großteil selbst verantwortlich 
waren (65,1\%). Mehr als die Hälfte der Teilnehmenden (57,9\%) dachte, dass sie zukünftig ein gesundes und positives Sexualleben haben würde (s. Abbildung 15).

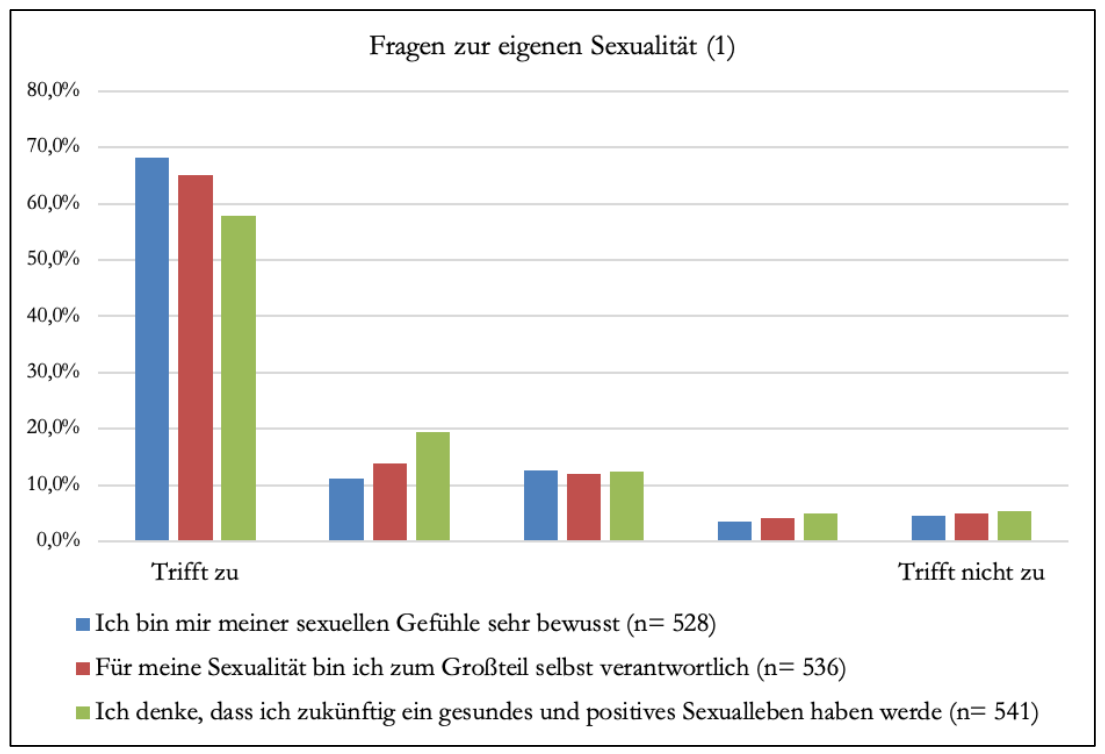

Abbildung 15 Fragen zur eigenen Sexualität (1)

Knapp ein Drittel der Teilnehmenden (33,2\%) befand, dass sie sich selbst nicht die Schuld geben würden, sollte etwas in ihrem Sexualleben schiefgehen. Mehrheitlich (53,7\%) meinten die Teilnehmenden, dass eine Bewertung ihres Sexualverhaltens und ihrer sexuellen Einstellung durch Mitmenschen für sie nicht wichtig sei. Für 24,5\% der Teilnehmenden traf es (eher) zu, dass ihr sexuelles Verhalten hauptsächlich durch ihre Mitmenschen bestimmt werde. 42,1\% der Befragten befanden, dass dies nicht auf sie zutreffen würde. Auch erachteten 41,7\% der Teilnehmenden, dass die Aussage „Mir fällt schnell auf, wie Mitmenschen die sexuellen Aspekte meines Lebens bewerten“ nicht auf sie zutreffe (s. Abbildung 16). 


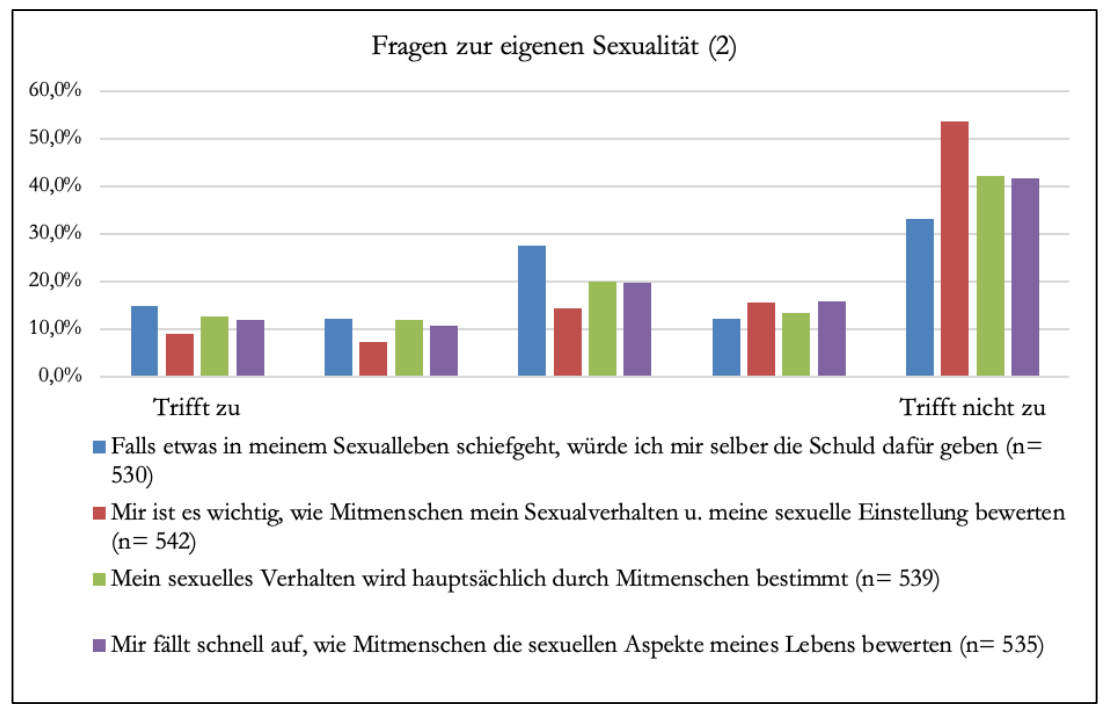

Abbildung 16 Fragen zur eigenen Sexualität (2)

\subsection{Q 5: Wie werden die Themen Sexualität im Elternhaus und Sexualaufklärung behandelt?}

Anhand einer fünfstufigen Likert-Skala wurden Fragen zum Thema Offenheit in der Familie beantwortet (Items 9.1 - 9.4). Dabei variierten die Angaben zwischen $1=$ Sehr häufig/Offen/Sehr offen und 5= Gar nicht häufig/Gar nicht offen. Die deskriptiven Darstellungen der Items sind der Abbildung 17 und Abbildung 18 zu entnehmen.

Generell zeigte sich eine eher restriktive Einstellung zum Thema Offenheit in der Familie (Items 9.2 - 9.4). Über die Hälfte der Studienteilnehmenden (51,3\%) berichtet, dass die eigenen Eltern „Gar nicht offen“ über ihre eigene Sexualität gesprochen hatten. 28,8\% der Befragten gab an, dass Sexualität „Gar nicht offen“ in der Familie angesprochen wurde. In 33,5\% der Fälle war ebenfalls „Gar nicht offen“ mit den eigenen Eltern über Sexualität gesprochen worden (s. Abbildung 17). Weniger als die Hälfte der Teilnehmenden ( $n=249$; 45,9\%) hatten eher weniger bis gar nicht häufig Sexualität in ihrer Familie thematisiert (s. Abbildung 18). 


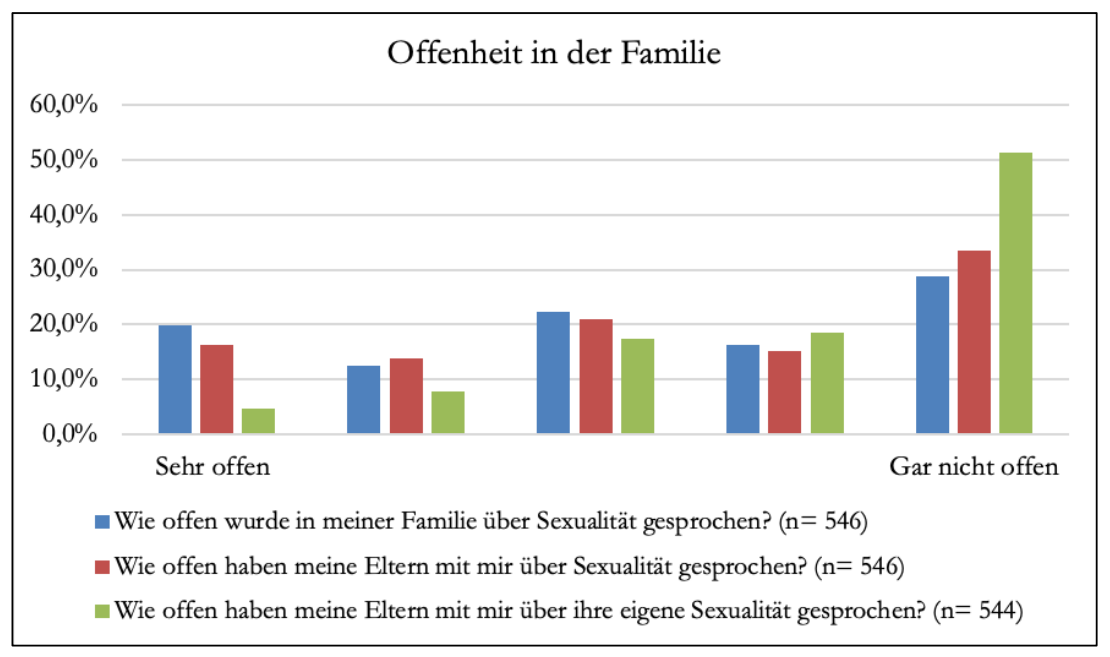

Abbildung 17 Offenheit in der Familie

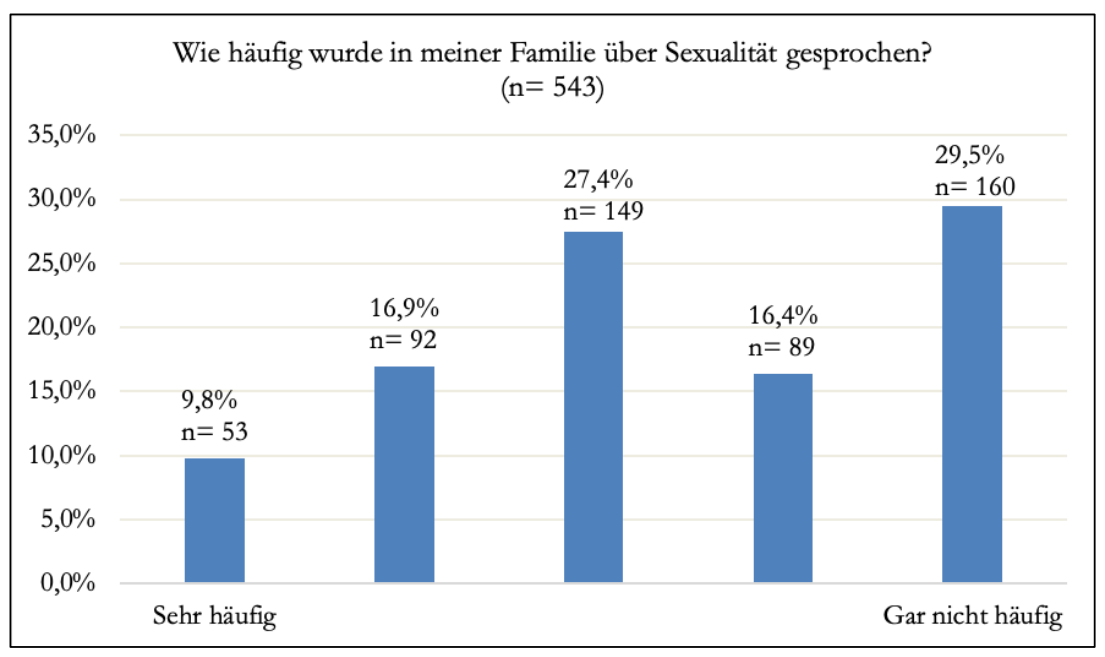

Abbildung 18 Wie häufig wurde in meiner Familie über Sexualität gesprochen? ( $n=543$ )

Ferner wurden Studienteilnehmende zu ihrer Sexualaufklärung befragt (s. Tabelle 17). Über die Hälfte der Teilnehmenden $(n=314 ; 52,5 \%)$ gibt an, vorwiegend in der Schule aufgeklärt worden zu sein. Wichtige Bezugspersonen in der Sexualaufklärung waren Freunde ( $n=274$; $45,8 \%)$ und die Mutter $(n=230 ; 38,5 \%)$. Ähnlich viele Nennungen erhielt das Internet als Hauptaufklärungsquelle ( $\mathrm{n}=211 ; 35,3 \%)$. Die Bezugsperson „Vater“ spielte eine vergleichsweise untergeordnete Rolle in der Sexualaufklärung ( $n=83 ; 13,9 \%)$. Unter Berücksichtigung der Geschlechterzugehörigkeit der Teilnehmenden (s. Tabelle 18) war für knapp die Hälfte der weiblichen Befragten die Mutter die Hauptaufklärungsquelle ( $n=136$; 48,1\%). Insgesamt zeigte sich sowohl bei den männlichen $(n=63 ; 53,8 \%)$ als auch bei den weiblichen ( $\mathrm{n}=172 ; 60,8 \%)$ Teilnehmenden die Institution Schule als Hauptaufklärungsort. Studienteilnehmende, die sich einem anderen Geschlecht zugehörig fühlten, wurden hauptsächlich durch ihre Freunde aufgeklärt $(n=6 ; 33,3 \%)$. 
Tabelle 17 Von wem bin ich hauptsächlich aufgeklärt worden? $(n=598)$

\begin{tabular}{ll}
\hline Aufklärung durch: & Anzahl $n(\%)$ \\
Fernsehen & $150(25,1)$ \\
Freunde & $274(45,8)$ \\
Geschwister & $99(16,6)$ \\
Internet & $211(35,3)$ \\
Mutter & $230(38,5)$ \\
Schule & $314(52,5)$ \\
Vater & $83(13,9)$ \\
Zeitschriften & $173(28,9)$ \\
Gar nicht & $43(7,2)$ \\
\hline
\end{tabular}

Tabelle 18 Von wem bin ich hauptsächlich aufgeklärt worden? Aufteilung nach Geschlechtszugehörigkeit

\begin{tabular}{llll}
\hline Aufklärung durch: & Männlich $n(\%)$ & Weiblich $n(\%)$ & Sonstige Zuordnung $n(\%)$ \\
Fernsehen & $30(25,6)$ & $70(24,7)$ & $3(16,7)$ \\
Freunde & $49(41,9)$ & $140(49,5)$ & $6(33,3)$ \\
Geschwister & $13(11,1)$ & $48(17)$ & $5(27,8)$ \\
Internet & $56(47,9)$ & $90(31,8)$ & $3(22,2)$ \\
Mutter & $40(34,2)$ & $136(48,1)$ & $5(27,8)$ \\
Schule & $63(53,8)$ & $172(60,8)$ & $3(16,7)$ \\
Vater & $28(23,9)$ & $35(12,4)$ & $3(16,7)$ \\
Zeitschriften & $20(17,1)$ & $104(36,7)$ & $2(11,1)$ \\
Gar nicht & $13(11,1)$ & $17(6)$ & $4(22,2)$ \\
Gesamt & $117(28)$ & $283(67,7)$ & $18(4,3)$ \\
\hline
\end{tabular}

Weiterhin wurden Studienteilnehmende befragt, welche Themen im Rahmen der elterlichen Sexualaufklärung besprochen wurden. Fast die Hälfte der Teilnehmenden ( $\mathrm{n}=290 ; 48,5 \%$ ) gab an, durch die Eltern über die monatliche Blutung (Periode) und über Verhütung ( $\mathrm{n}=$ 295; 49,3\%) aufgeklärt worden zu sein. Auch die Themen Schwangerschaft ( $\mathrm{n}=281 ; 47 \%)$ und Geburt ( $\mathrm{n}=263 ; 44 \%$ ) wurden häufig als Aufklärungsthemen genannt. Am wenigsten wurde das Thema „Sexuelle Funktionsstörungen“ ( $\mathrm{n}=55 ; 9,2 \%)$ besprochen. Fast ein Fünftel der Teilnehmenden $(n=118 ; 19,7 \%)$ gab an, dass sie gar nicht durch ihre Eltern aufgeklärt worden sind (s. Tabelle 19). 
Tabelle 19 Über Folgendes bin ich von meinen Eltern aufgeklärt worden $(\mathrm{n}=598)$

\begin{tabular}{ll}
\hline Thema der Aufklärung: & Anzahl $n(\%)$ \\
Geburt & $263(44)$ \\
Geschlechtskrankheiten & $206(34,4)$ \\
Heterosexualität & $118(19,7)$ \\
Homosexualität & $117(19,6)$ \\
Monatliche Blutung (Periode) & $290(48,5)$ \\
Schwangerschaft & $281(47)$ \\
Selbstbefriedigung & $76(12,7)$ \\
Sexuelle Funktionsstörungen & $55(9,2)$ \\
Verhütung (Pille, Kondome, Spirale ...) & $295(49,3)$ \\
Gar nicht & $118(19,7)$ \\
\hline
\end{tabular}

Insgesamt befand die Mehrheit der Studienteilnehmenden, dass sie (sehr) gut aufgeklärt sei $(n=375 ; 71,2 \%)$. Lediglich ein geringer Teil $(n=51 ; 9,7 \%)$ der Befragten stufte sich selbst als gar nicht aufgeklärt ein (s. Abbildung 19).

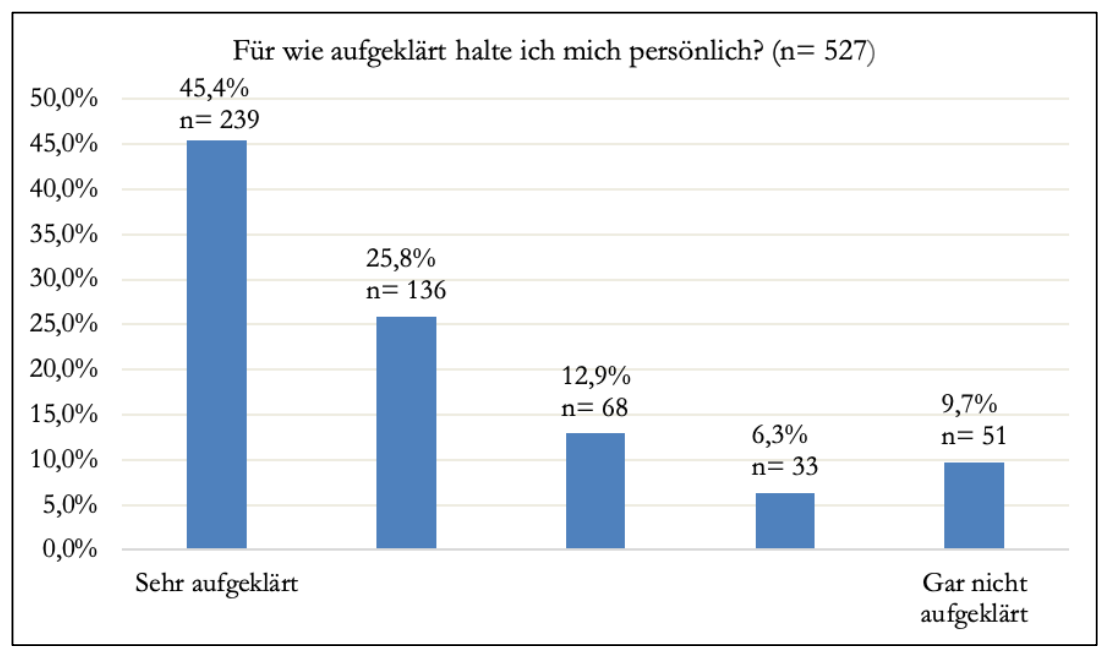

Abbildung 19 Für wie aufgeklärt halte ich mich persönlich? $(\mathrm{n}=527)$ 


\section{Diskussion}

In der vorliegenden Arbeit wurden Altenpflegende und Auszubildende hinsichtlich ihrer Erfahrungen zu den Themen sexuelle Gesundheit und Alterssexualität in ihrem Berufsalltag befragt. Ziel der Studie war es, eine Bestandsaufnahme im Sinne einer subjektiven Einschätzung zu diesen Themen zu erheben. Da es sich um subjektive Eindrücke der Teilnehmenden handelt, können reale Aussagen weder über das Verhalten noch über die Einstellungen von Heimbewohnenden getroffen werden. Im Folgenden werden die wichtigsten Ergebnisse diskutiert. Hierbei werden die Fragestellungen (Q) im Zusammenhang mit dem (aktuellen) Forschungsstand sowie der einschlägigen Literatur betrachtet. Insgesamt gibt es nur wenig deutschsprachige Studien und Literatur zu diesem Studienthema. Vergleichend werden daher auch nicht-deutsche Studien hinzugezogen. Da diese auf unterschiedlichen Ausbildungs- und Gesundheitssystemen beruhen, ist ein direkter Vergleich der Studienergebnisse mit internationalen Studien nur eingeschränkt möglich.

Im Rahmen der Studienergebnisse muss bedacht werden, dass es durch die Teilnahmebereitschaft oder Ablehnung der Schul- oder Pflegedienstleitungen zu einer Beeinflussung der Studienergebnisse kommen könnte. So ist zu vermuten, dass Leitungspositionen, bei denen ein Bewusstsein für das Thema Alterssexualität besteht, tendenziell eine höhere Bereitschaft zur Studienteilnahme geboten hatten. Unabhängig von der Einstellung der Leitungspersonen muss berücksichtigt werden, dass die Studienteilnahme des Einzelnen ebenfalls freiwillig erfolgte. Demnach war auch die individuelle Bereitschaft, sich auf die Thematik einzulassen, von Bedeutung. Durch eine Teilnahmebereitschaft oder Ablehnung der Schul- oder Pflegedienstleitungen sowie des individuellen Befragten kann es im Sinne eines Selection Bias zu einer Verzerrung der Ergebnisse kommen (vgl. Herkner und Müllner 2011). Dieser Bias entsteht, wenn Befragte, insbesondere bei heiklen Themen wie Sexualität, Fragen nicht oder nicht wahrheitsgemäß beantworten (vgl. Herkner und Müllner 2011). Ebenso ist vorstellbar, dass zeitliche und personelle Herausforderungen des Pflegealltags (bspw. Zeit- und Fachkräftemangel) die Teilnahmebereitschaft beeinflussen.

\subsection{Stichprobe}

Initiales Bestreben der Studie war es, dass sowohl examinierte Altenpflegende als auch Befragte aus verschiedenen Ausbildungsjahren in repräsentativer Anzahl an der Studie teilnahmen. Eine möglichst ausgewogene Alters- und Ausbildungsstruktur der Teilnehmenden wäre für die Studie wünschenswert gewesen. Allerdings war aufgrund des 
hohen Rücklaufs von den Schulen die Gruppe der Auszubildenden (81,3\%) im Vergleich zu der Gruppe der examinierten Altenpflegenden (14,5\%) deutlich überrepräsentiert. Die Daten des Statistischen Bundesamtes Wiesbaden (2017) zeigten, dass nur rund 7\% der Beschäftigten in Altenpflegeheimen Auszubildende waren. Entsprechend dem Ausbildungsstand war auch die Altersverteilung nicht repräsentativ zur Grundgesamtheit der Altenpflegenden. In der vorliegenden Arbeit machte die Altersgruppe $<25 \mathrm{Jahren}$ mit einem Anteil von 39,3\% den Großteil der Studienteilnehmenden aus (vgl. Tabelle 4). Die Altersstruktur der Pflegestatistik 2015 gab an, dass 18\% der Arbeitenden in Pflegeheimen unter 30 Jahre alt waren (Statistisches Bundesamt 2017). Zwischen 30 bis 49 Jahren alt waren 41\% der Beschäftigten und 50 Jahre und älter waren 40\% (Statistisches Bundesamt 2017). Daher können die Ergebnisse der vorliegenden Studie nur mit Einschränkungen auf die Gesamtheit der Altenpflegenden und Auszubildenden der Altenpflege übertragen werden. Weiterhin könnte es durch den geringen Anteil an Altenpflegenden mit einer abgeschlossenen Ausbildung zu einer Verschiebung der Ergebnisse gekommen sein. Dies würde insbesondere auf Fragen zutreffen, die sich mit im Pflegealltag erlebten Situationen oder Ausbildungsinhalten auseinandersetzten. So sind Auszubildende der Altenpflege möglicherweise nicht in alle Abläufe der Bewohnerversorgung integriert. Dementsprechend muss bedacht werden, dass eine ältere und in der Ausbildung weiter fortgeschrittene Stichprobe mit mehr Berufserfahrung eventuell zu anderen Ergebnissen hätte führen können (vgl. Bouman et al. 2007; Bauer et al. 2013).

Bei Betrachtung des Alters und der Einstellung zur Sexualität zeigten sich insbesondere die jüngeren Teilnehmenden der vorliegenden Studie toleranter in ihren Antworten (s. Tabelle 16). Die Altersgruppe der über 60-Jährigen beantwortete Items zum Thema Sexualität im Alter generell restriktiver als die jüngeren Altersgruppen. Dennoch gilt es, in diesem Zusammenhang die Größe der Stichprobe zu berücksichtigen. Die Gruppe der > 60Jährigen machte nur 4,9\% der Gesamtstichprobe aus und könnte daher bei fehlender Repräsentanz zu einer Verzerrung der Aussagen führen. Im Gegensatz zu den Beobachtungen der vorliegenden Arbeit beschrieben Bouman et al. (2007), dass jüngeres Pflegepersonal das Thema Alterssexualität negativer und restriktiver bewertete als ältere Pflegende mit mehr Berufserfahrung. Die Autoren halten für sehr wahrscheinlich, dass der geringere Altersunterschied zu einer größeren Toleranz der älteren Pflegenden gegenüber den Bewohnenden führen könnte, da sie sich möglicherweise eher in die Lage der Bewohnenden hineinversetzen könnten (vgl. Bouman et al. 2007). Demgegenüber fanden sich bei Bauer et al. (2013) keine Unterschiede in der Bewertung von Alterssexualität im Zusammenhang mit dem Alter oder der Arbeitserfahrung der Teilnehmenden. Daher lässt 
die Zusammenschau der Studienergebnisse vermuten, dass keine eindeutigen Aussagen über die Einstellung von Alterssexualität und das Alter der Befragten zu treffen sind.

\subsection{Erhebungsinstrument}

Im Folgenden wird der Fragebogen als Instrument der Datenerhebung kritisch diskutiert. Um an einer Befragung teilnehmen zu können, durchläuft der Befragte verschiedene kognitive Prozesse (vgl. Porst 2014; Krosnick und Presser 2009; Krosnick et al. 1996; Bogner und Landrock 2015). Zuerst muss die Frage verstanden und die zur Beantwortung relevanten Informationen aus dem Gedächtnis abgerufen werden. Auf der Basis dieser Informationen wird ein Urteil gebildet und dieses Urteil ggf. an das vorgeschriebene Antwortformat angepasst. Vor der endgültigen Beantwortung der Frage muss das private Urteil ggf. noch an die Meinung des Fragenden bzw. des Fragebogens adaptiert werden (Porst 2014; Krosnick und Presser 2009; Krosnick et al. 1996; Bogner und Landrock 2015).

Für den ersten Schritt vor der Beantwortung einer Frage beschreibt Porst (2014), dass diese sowohl auf semantischer (Was soll das ,heißen"?) als auch auf pragmatischer Ebene (Was will mein Gegenüber ,wissen“?) verstanden werden muss. In der vorliegenden Studie wurden einige Items (Items 5.2 - 5.5) in ihrem Antwortformat bewusst zeitlich nicht festgelegt formuliert (Nie/Gelegentlich/Regelmäßig), um die subjektiven Einstellungen der Teilnehmenden zu erfassen. Auch die Begriffe „körperliche“ oder „,verbale sexuelle Übergriffe“ (Items 5.6 5.11) wurden absichtlich nicht genau definiert, um die subjektive Einschätzung der Befragten zu diesen Themen zu erhalten. Diese Items liefen aufgrund ihrer offeneren Formulierung Gefahr, auf pragmatischer Ebene nicht verstanden zu werden (vgl. Porst 2014). Aufgrund von fehlendem Verständnis hätte es daher bspw. zum Auslassen oder zur FalschBeantwortung von Fragen kommen können (vgl. Porst 2014). Um dem vorzubeugen, war der Fragebogen vor Studienbeginn durch verschiedene Berufsgruppen auf Verständnis geprüft worden (s. Kapitel 2.4.2).

Der kognitive Aufwand, den Befragte beim Beantworten von Fragen aufbringen müssen, kann in die Begriffe Optimizing und Satisficing unterschieden werden (vgl. Krosnick et al. 1996; Krosnick und Presser 2009; Bogner und Landrock 2015). Beim Optimiz̨ing durchläuft der Befragte die vier o. g. Schritte, um eine Frage mit ausreichend kognitivem Aufwand zu beantworten (vgl. Krosnick et al. 1996; Krosnick und Presser 2009; Bogner und Landrock 2015). Das Satisficing kann in schwach (der Befragte durchläuft zwar alle vier Schritte, reduziert aber den kognitiven Aufwand) und stark (der Befragte verkürzt die Informationsverarbeitung, evtl. sogar bis zum Nichtlesen der Fragen) unterschieden werden (vgl. Krosnick et al. 1996; Krosnick und Presser 2009; Bogner und Landrock 2015). Zeigen 
Befragte ein Verhalten von Satisficing, so besteht bei manchen Fragen die Gefahr, nur oberflächlich beantwortet zu werden (Krosnick et al. 1996; Krosnick und Presser 2009; Bogner und Landrock 2015). Ob ein Befragter die Satisficing-Strategie wählt, hängt laut Krosnick et al. (1996) im Wesentlichen von drei verschiedenen Faktoren ab: der Aufgabenschwierigkeit, der kognitiven Fähigkeit des Befragten, diese Aufgaben zu erfüllen, und der Motivation des Befragten, die Aufgaben durchzuführen. Je schwieriger die Aufgabe im Vergleich zur Fähigkeit und Motivation des Befragten ist, desto eher besteht die Tendenz zum Satisficing (Krosnick et al. 1996; Krosnick und Presser 2009; Bogner und Landrock 2015). Die Auswirkung dieser Prozesse kann zu Antworttendenzen führen (Bogner und Landrock 2015).

Eine Form von Antworttendenz in Umfragen ist die soziale Erwünschtheit (Bogner und Landrock 2015). Sie beschreibt vom soziologischen Standpunkt aus ein Antwortverhalten, das sowohl von Inhalten als auch von Situationen abhängig ist und eine Variation innerhalb gesellschaftlicher Untergruppen aufzeigt (Krebs 1991). Erklärt man den Begriff der sozialen Erwünschtheit aus psychologischer Sicht, werden positive Merkmale zur Selbstdarstellung im Vergleich zu den negativen tendenziell überschätzt (Krebs 1991). Dennoch scheint das Verhalten von sozialer Erwünschtheit nicht gleichmäßig in der Gesellschaft etabliert zu sein (vgl. Krebs 1991). Zwar scheint die Tendenz zwischen Männern und Frauen gleich ausgeprägt, sie variiert aber abhängig vom Alter, Familienstand, Schulbildung und Gehalt des Befragten (Krebs 1991). Aufgrund von mangelnder Erfahrung mit gesellschaftlich anerkantem Antwortverhalten zeigte in einer Studie die Gruppe der 18- bis 29-Jährigen die geringste Tendenz zur sozial erwünschten Antwort (Krebs 1991).

Ein Vorteil der vorliegenden Studie war die Datenerhebung mittels Fragebogen im Vergleich zur Interviewbefragung, womit die Wahrscheinlichkeit einer situativen bzw. sozial erwünschten Antwort verringert wurde (vgl. Bogner und Landrock 2015). Die Tendenz zur sozialen Erwünschtheit ist größer, wenn die soziale Distanz - bspw. im Falle eines Interviews durch physische Nähe - gering ist. Denn hier läuft der Befragte Gefahr, sich vor dem Interviewer bloßzustellen (Bogner und Landrock 2015). Bei einer größeren Distanz, z. B. im Rahmen einer anonymen Fragebogenstudie, besteht eine geringere Tendenz zur positiven Selbstdarstellung (vgl. Bogner und Landrock 2015). Dennoch hätte es trotz der Verwendung eines Fragebogens in der vorliegenden Studie aufgrund von sozialer Erwünschtheit zu einer Verzerrung des Antwortverhaltens kommen können (vgl. Krebs 1991). Die Ergebnisse deuten darauf hin, dass Teilnehmende zumindest teilweise, sowohl in der psychologischen als auch in der soziologischen Auslegung des Begriffs, zu sozial erwünschten Antworten neigen. Diese Vermutung entsteht insbesondere bei Items, die das eigene Sexualverhalten 
porträtieren (Items 8.1, 8.7 - 8.12), aber auch bei Fragen zur Akzeptanz der Sexualität anderer Menschen (Items 7.13, 8.2 - 8.5). Allerdings deuten die Ergebnisse der von Krebs (1991) beschriebenen Studie darauf hin, dass zumindest aufgrund des niedrigen Alters der Teilnehmenden der vorliegenden Studie das Antwortverhalten insgesamt eher die subjektive Meinung der Befragten wiedergibt (vgl. Krebs 1991). Dennoch können insgesamt im Rahmen der vorliegenden Studie nur Vermutungen angestellt werden, ob und inwieweit soziale Erwünschtheit einen Einfluss auf die Beantwortung der Items hatte (s. Kapitel 4.6).

Ein Nachteil bei der Verwendung eines Fragebogens ist, dass Studienteilnehmende dazu tendieren, nicht alle vorgegebenen Fragen zu beantworten (vgl. Herkner und Müllner 2011). Möglicherweise war die hohe Anzahl an Items (Testlänge) nachteilig, sodass einzelne Items von Studienteilnehmenden eventuell aus diesem Grund weggelassen wurden (vgl. Porst 2014). Auch mangelnde oder nachlassende Konzentration sowie Desinteresse könnten eine Ursache für das Weglassen bzw. der Falsch-Beantwortung von Items sein (vgl. Krosnick et al. 1996). Generell ist davon auszugehen, dass Items, die sich mit der persönlichen Einstellung zum Thema Sexualität befassten, Gefahr liefen, gar nicht oder nicht wahrheitsgemäß beantwortet zu werden (vgl. Herkner und Müllner 2011).

Neben der Tendenz zur sozialen Erwünschtheit lässt auch das Hinzufügen der Antwortoption „Weiß nicht“ zu den initial dichotomen Antwortmöglichkeiten (s. Kapitel 2.4.2) Raum für Interpretation (vgl. Franzen 2014). Laut Franzen (2014) beinhaltet die Kategorie „Weiß nicht“ sowohl eine echte Meinungslosigkeit - sie birgt aber auch das Risiko, dass Befragte sie wählen, um bei der Beantwortung von Fragen den „kognitiven Aufwand zu reduzieren, um eine Frage zu verstehen und zu beantworten“. Diese Beobachtung spiegelt sich in den Wissensfragen (s. Kapitel 4.5) und in Items wider, die das Thema Sexualassistenz (s. Kapitel 4.4) behandeln. Gegebenenfalls wird die Antwortoption „Weiß nicht“ auch ausgewählt, wenn Teilnehmende ihre Meinung aufgrund von Fehlen der „richtigen“ Antwortoption nicht zum Ausdruck bringen können.

Im Rahmen der Studie wurde keine zeitliche Begrenzung bei der Beantwortung der Fragen vorgegeben. Aber es ist anzunehmen, dass die Fragebögen von den Studienteilnehmenden während ihrer Arbeitsschicht im Heim bzw. während der Schulzeit ausgefüllt wurden, so dass sich eine zeitliche Begrenzung bei der Ausarbeitung nicht ausschließen lässt. So könnte es aufgrund von Zeitdruck ebenfalls zum Auslassen von Fragen gekommen sein.

In Zusammenschau der Beobachtungen gibt es multiple Gründe, warum einzelne Teilnehmende zur Nicht-Beantwortung, zum Auslassen oder zur „Falsch-Beantwortung“ 
von Items tendieren. Im Rahmen dieser Studie können diese jedoch nicht abschließend geklärt werden und unterliegen nur Vermutungen.

\subsection{Q 1: Welchen Stellenwert hat das Thema Sexualität und sexuelle Gesundheit in der Altenpflegeausbildung?}

Die Ergebnisse der vorliegenden Studie deuten auf eine Diskrepanz zwischen der subjektiv empfundenen Wichtigkeit der Thematik Alterssexualität und der eigentlichen Umsetzung des Themas im Lehr- und Berufsalltag hin. Zum Zeitpunkt der Befragung gaben weniger als die Hälfte ( $n=243 ; 41,7 \%$ ) der Studienteilnehmenden an, adäquat das Thema Alterssexualität während ihrer Altenpflegeausbildung behandelt zu haben. Einschränkend muss beachtet werden, dass zum Erhebungszeitpunkt nicht alle Studienteilnehmenden der vorliegenden Studie examiniert war. Somit besteht die Möglichkeit, dass Alterssexualität erst zu einem späteren Ausbildungszeitpunkt behandelt wird. Zudem deuten die Ergebnisse des befragten Lehrenden-Kollektivs darauf hin, dass zumindest ein Teil der Auszubildenden im Verlauf ihrer Ausbildung noch über das Thema Alterssexualität unterrichtet werden wird (s. Kapitel 3.1). Hierfür spricht, dass $78,3 \%$ der befragten Lehrenden Sexualität im Alter als Pflichtbestandteil des Curriculums angaben.

Vergleichend zeigte sich in einer Londoner Studie von Lewis und Bor (1994), dass 68,1\% der befragten Krankenpflegenden während ihrer Grundausbildung über Sexualität unterrichtet wurden. Dennoch ist zu beachten, dass die Stichprobe der Studie von Lewis und Bor kleiner ( $\mathrm{n}=161)$ war und daher weniger aussagekräftig sein könnte. Somit könnte es unter Berücksichtigung des unterschiedlichen Ausbildungsstandes und der Stichprobengröße zu einer Annäherung der Ergebnisse beider Studien kommen. Zudem muss bei der Betrachtung der Ergebnisse bedacht werden, dass die Unterrichtung des Themas Alterssexualität abhängig von der Altenpflegeschule sowie den Lehrenden ist. Dies gilt insbesondere, da zum Thema Alterssexualität in den Rahmenrichtlinien für den berufsbezogenen Lernbereich in der Berufsfachschule-Altenpflege keine verbindlichen Angaben gemacht werden (vgl. Niedersächsisches Kultusministerium 2003). Trotz der geringen Thematisierung hielt ein Großteil der befragten Auszubildenden/Examinierten ( $n=390 ; 68,5 \%)$ das Thema Alterssexualität für ein für den Arbeitsalltag relevantes Ausbildungsfach (s. Abbildung 7). In Zusammenschau der o. g. Ergebnisse scheint es daher wichtig zu sein, dass nicht nur die Quantität von sexueller Bildung im schulischen Kontext eine Rolle spielt, sondern dass gelernte Inhalte eine Alltagsrelevanz aufweisen müssen. 
Um die Frage nach Alterssexualität als Ausbildungsinhalt eindeutiger zu untersuchen, werden im Folgenden die Aussagen der Lehrenden betrachtet. Da die Stichprobe der Lehrenden klein ist $(n=30)$, muss sie als nicht repräsentativ für alle Altenpflegeschulen angesehen werden. Ebenfalls ist durch die Anonymisierung der Fragebögen kein Rückschluss auf die einzelnen Schulen möglich, womit die Lehrqualität dieser nicht differenziert werden kann.

66,7\% $(n=20)$ der befragten Lehrenden gaben an, didaktische Fähigkeiten zur Vermittlung des Themas Alterssexualität erwerben zu wollen. Dieses Ergebnis deutet darauf hin, dass die befragten Lehrenden das Thema Alterssexualität als ausbildungsrelevant einstufen und fundiert auf die Unterrichtung des Themas vorbereitet sein wollen. Im Gegensatz zu der insgesamt recht positiven Einstellung der in der vorliegenden Studie befragten Lehrenden stellte Sielert (2011) fest, dass ein Großteil der Lehrenden während der allgemeinen Schulausbildung nicht gerne auf das Thema Sexualerziehung eingeht und auch nicht genügend auf das Unterrichten des Themas vorbereitet wird. Dennoch muss bei einem Vergleich beider Studien berücksichtigt werden, dass es sich in der Studie von Sielert (2011) um die Lehrenden von Grundschülern handelt und daher aufgrund des jungen Alters der Schüler eventuell andere Hemmschwellen durch die Lehrenden überwunden werden müssen. Darüber hinaus kritisiert Schmidt (2014), dass es an Schulen an gut ausgebildeten, sensibilisierten Lehrenden mangelt, welche den wachsenden Ansprüchen der schulischen Sexualpädagogik nicht gerecht werden. Zu Lösungen in diesem Zusammenhang tragen nicht nur gut ausgebildete Lehrkräfte bei, sondern auch standardisiertes Lehrmaterial, das eine positive Herangehensweise an das Thema Sexualität aufzeigt (Leitz und Signerski-Krieger 2018). Angesichts der Besonderheiten bei der Sexualerziehung werden zudem spezifische methodische Kompetenzen der Lehrenden gefordert (vgl. Sielert 2015).

\subsection{Q 2: Welchen Stellenwert hat das Thema Sexualität und sexuelle Gesundheit im Pflegealltag?}

\section{Konzept Sexualassistenz}

Bei der Sexualassistenz handelt es sich um eine sexuelle Dienstleistung, die Menschen mit körperlicher und/oder psychischer Beeinträchtigung in der Verwirklichung bzw. dem Ausleben ihrer Sexualität unterstützt (vgl. de Vries 2011). Insgesamt zeigten die Ergebnisse der vorliegenden Studie, dass das Konzept Sexualassistenz vielen der Teilnehmenden unbekannt war. Nur 12,7\% ( $\mathrm{n}=73)$ der Studienteilnehmenden hatten eine Umsetzung in ihrer Berufslaufbahn erlebt. Unter den Teilnehmenden schien es keine eindeutige Meinung bezüglich einer Befürwortung von Sexualassistenz im Pflegealltag zu geben. Zudem wurden die Fragen häufig mit der Antwortoption „Weiß nicht“ beantwortet, was darauf hindeuten 
kann, dass zum Thema Sexualassistenz Unsicherheiten bestehen. Die Autorin und Sexualassistentin Nina de Vries (2011) beschreibt, dass die Sexualassistenz keinen klar etablierten Stellenwert in der jetzigen Pflegesituation hat. Laut de Vries (2011) zählen bezahlte sexuelle Dienste generell zu den gesellschaftlich tabuisierten Themen. Somit vereint das Konzept der Sexualassistenz sowohl die gesellschaftlichen Tabuthemen Sexualität und Pflege als auch das Tabu der bezahlten sexuellen Dienstleistung in sich (vgl. de Vries 2011). Daher sei eine aktive Sexualassistenz nur möglich, wenn die eigene Sexualität im Vorhinein klar reflektiert wurde (vgl. de Vries 2011). Weiterhin müssen Heime Strukturen entwerfen, die den Pflegenden Sicherheit auf dem Gebiet der Sexualität in der Bewohnerversorgung verschaffen und den Bewohnenden das aktive Ausleben von Sexualität ermöglichen (de Vries 2011). Unklar bleibt im Rahmen dieser Studie, ob Teilnehmende eine wirkliche Meinungslosigkeit bzw. ihre Unsicherheit zum Ausdruck bringen wollten, oder ob die Option „Weiß nicht“ als Ausdruck eines reduzierten kognitiven Aufwandes bei der Beantwortung der Items verwendet wurde (vgl. Franzen 2014). Sollten die Ergebnisse ein Ausdruck von Unsicherheit sein, so scheinen die von de Vries beschriebenen Lösungsansätze noch keine eindeutige Präsenz im Pflegealltag zu zeigen.

\section{Fortbildungsmöglichkeit am Arbeitsplatz}

Eine Vielzahl der Teilnehmenden ( $\mathrm{n}=396 ; 71,9 \%)$ wünscht sich eine Fortbildung zum Thema Alterssexualität und sexuelle Gesundheit. Fast 60\% der befragten Examinierten und Auszubildenden ( $\mathrm{n}=350 ; 58,5 \%$ ) wollen im Rahmen von Fortbildungen mehr über den Umgang mit sexuell aktiven Heimbewohnern lernen. Insgesamt lässt der Fortbildungswunsch der Teilnehmenden vermuten, dass Alterssexualität und sexuelle Gesundheit einen aktiven Stellenwert im Berufsalltag haben und dass die Befragten mehr zu den Themen erfahren wollen. Dass es durch Fortbildungen zumindest kurzfristig zu einer Änderung der Einstellung gegenüber der Sexualität älterer Menschen kommen kann, konnte eine australische Studie anhand eines Pre- und Posttests zeigen (vgl. Bauer et al. 2013). So ergab der Posttest eine signifikant positive Auswirkung auf die Toleranz und die Einstellung der Befragten zum Thema Alterssexualität. Die Autoren diskutierten jedoch, dass nur der kurzfristige Effekt der Fortbildung beobachtet werden konnte. Im Rahmen der Studie konnte nicht evaluiert werden, ob sich diese positive Veränderung auch in der praktischen pflegerischen Tätigkeit widerspiegelt (vgl. Bauer et al. 2013). Dennoch scheinen Fortbildungen gute Möglichkeiten zu bieten, ein besseres Arbeitsumfeld für Altenpflegende sowie eine daraus resultierende verbesserte Lebensqualität für Heimbewohnende zu erreichen, sofern sich eine positive Einstellung auch auf die praktischen Tätigkeiten eines 
Altenpflegenden auswirkt (vgl. Bauer et al. 2013). In einer Metaanalyse berichten Hillman und Stricker (1994), dass vermehrtes Wissen über Sexualität zu gesteigerter Toleranz gegenüber Alterssexualität führen kann. Dennoch gab es Wissenschaftler, die einen negativen oder nicht-signifikanten Zusammenhang der beiden Komponenten diskutierten (vgl. Hillman und Stricker 1994). Beispielsweise fanden Glass et al. (1986) in ihrer Studie heraus, dass vermehrtes Wissen zum Thema Alterssexualität eher zu restriktiveren Einstellungen gegenüber Alterssexualität führte. Die Zusammenschau der Ergebnisse deutet auf die Komplexität der Interdependenz von Wissen und Einstellung zu Alterssexualität hin.

\section{Situation in meiner Einrichtung/meinem Wohnbereich}

Die Ergebnisse der vorliegenden Arbeit lassen vermuten, dass in Altenpflegeheimen unzureichend auf die Privatsphäre von Heimbewohnenden geachtet wird. Dem subjektiven Erleben der Teilnehmenden nach gibt es wenig Raum für das ungestörte Ausleben von Sexualität in Pflegeeinrichtungen. So hatten 45,8\% $(n=259)$ der Studienteilnehmenden bereits sexuelle Aktivität unter Heimbewohnenden erlebt. Zudem berichten die Befragten, dass es zwar Vorkehrungen gibt, die die Privatsphäre der Bewohnenden schützen, dennoch scheinen diese nicht vollständig etabliert zu sein. Verschiedene Beobachtungen in der Literatur sprechen ebenfalls die mangelnde Privatsphäre der Heimbewohnenden an (vgl. Sieren 2012; Grond 2001; Zettl-Wiedner 2011). In einer Fragebogenstudie von ZettlWiedner (2011) beurteilten die Bezugspflegepersonen die Sexualität der Bewohnenden in Langzeitpflegesituationen. Demnach wünschte sich fast die Hälfte der Heimbewohnenden (49,3\%) mehr Privat- und Intimsphäre (Zettl-Wiedner 2011).

Ein weiterer wichtiger Aspekt der vorliegenden Arbeit war es, sexuelle Gewalterfahrungen im Berufsalltag zu erfragen. Studienteilnehmende wurden nach der Häufigkeit von Situationen gefragt, die mit körperlichen oder verbalen sexuellen Übergriffen einhergingen. Knapp ein Fünftel der Studienteilnehmenden erlebten körperliche und/oder verbale sexuelle Gewalt, die von Bewohnenden ausging (s. Abbildung 8, Abbildung 9 und Abbildung 10). Zudem wünschen sich 42,0\% ( $\mathrm{n}=226)$ der Studienteilnehmenden eine offizielle Anlaufstelle, um über sexuelle Probleme/Vorkommnisse in der Bewohnerversorgung zu reden. Diese Beobachtungen sprechen dafür, dass sexuelle Gewalt einen aktiven Stellenwert im Pflegealltag hat. Im Einklang mit dem Studienergebnis der vorliegenden Studie werden sowohl sexuelle Übergriffe als auch andere Gewaltformen weltweit in Pflegeberufen beobachtet (vgl. Aström et al. 2002; Bronner et al. 2003; Sieren 2012). Eine schwedische Fragebogenstudie von Aström et al. (2002) zeigte, dass unter allen befragten Pflegeberufen 
die größte Inzidenz von Gewalt (inklusive sexueller Gewalt) gegen Mitarbeitende in Altenpflegeheimen stattfand. Im Rahmen einer Umfrage unter israelischen Krankenpflegenden fiel auf, dass insbesondere Krankenschwestern und weibliche Auszubildende sexueller Belästigung ausgesetzt waren (vgl. Bronner et al. 2003). Hingegen wurden männliche Pflegende zwar weniger oft sexuell belästigt, allerdings wurden sie mit schweren Formen der Belästigung, bspw. intimen Berührungen oder körperlichen sexuellen Annäherungsversuchen konfrontiert (vgl. Bronner et al. 2003). In Anbetracht der dargelegten Studienergebnisse fühlten sich nur 57,2\% der Teilnehmenden der vorliegenden Studie durch ihre Ausbildung gut auf verbal und körperlich sexuell übergriffiges Verhalten von Heimbewohnenden vorbereitet (s. Abbildung 5). Das vorwiegend geringe Alter sowie der Ausbildungsstand der Studienteilnehmenden suggeriert, dass Gewaltprävention und auch spezifische Prävention gegen sexuelle Übergriffe schon in der Ausbildung thematisiert werden und am Arbeitsplatz weiter eine Rolle spielen sollten.

Die Studienteilnehmenden der vorliegenden Studie berichten, dass sexuelle Gewalt nicht nur von Gepflegten, sondern auch von Pflegenden ausgeht (s. Abbildung 9). Die Beweggründe für sexuelle Übergriffe gegen Heimbewohnende wurden in der vorliegenden Studie nicht geklärt. Eine Erklärung zu verschiedenen Aspekten von Gewalt in der Pflege sucht die Autorin Esther Matolycz (2011). Sie erläutert das Prinzip der personalen Gewalt, welche beispielsweise durch die Missachtung der Intimsphäre, Beschimpfung von Heimbewohnenden oder Entmündigung durch Behandlung des Bewohnenden wie ein Kind ausgeübt wird. Achtsamkeit und Einfühlungsvermögen seien wichtig, um Handlungen in der Pflege zu entschärfen, die von Bewohnenden als gewaltvoll empfunden werden können. Durch diese Handlungen könne „akzidentelle“ Gewalt vermieden werden. Aggressionen, die Pflegende verspüren, verhindern Empathie, was zu absichtlichen gewalttätigen Handlungen gegen Bewohnende führen kann. Ursächlich für Aggressionen seien bspw. Zeitmangel und Überlastung im Pflegealltag. Aus diesem Grund rät die Autorin dazu, dass Pflegende die Kommunikation im Team als „Gewaltprophylaxe“ aufrechterhalten und besprechen, welche Heimbewohnende als besonders fordernd erlebt werden (Matolycz 2011).

\section{Situationen in meinem Berufsalltag}

Die Ergebnisse zu der Bereitstellung von sexuellen Hilfsmitteln in der Altenpflege zeigen, dass nur wenige Hilfsmittel in Einrichtungen zur Verfügung standen (Keine: $n=91 ; 66,1 \%$, s. Tabelle 12). Die meisten Nennungen erhielten Videos und Filme mit pornographischen Inhalten $(n=12 ; 3,2 \%)$, gefolgt von Vibratoren $(n=6 ; 1,6 \%)$. Ebenso gaben einige der 
Teilnehmenden an, dass Bewohnende Alltagsgegenstände wie Fernbedienungen, Löffel oder Schnabeltassen zur sexuellen Stimulation verwendeten. Die meisten Befragten ( $n=139$; 36,8\%) der vorliegenden Studie gaben an, nicht zu wissen, ob die Bereitstellung sexueller Hilfsmittel durch die Pflegeeinrichtung sinnvoll wäre. Dennoch antworteten fast ebenso viele $(n=140 ; 37 \%)$, dass eine Bereitstellung sinnvoll wäre, um ältere Menschen in ihrer sexuellen Aktivität zu unterstützen. Die Beobachtungen der vorliegenden Studie stimmen mit denen einer Interviewstudie überein, bei denen Altenpflegende beobachtet hatten, wie Bewohnende beispielsweise Haarklammern zur sexuellen Stimulation verwendeten (vgl. Sieren 2012). Die Teilnehmenden berichteten von dem Zwiespalt, die Privatsphäre des Bewohnenden respektieren zu wollen, andererseits ggf. sexuelle Hilfsmittel für diese zu beschaffen, um das Verletzungsrisiko (bspw. beim Gebrauch von Haarklammern) zu reduzieren (vgl. Sieren 2012).

Insgesamt zeigen die Ergebnisse der vorliegenden Studie, dass wenig „offizielle“ sexuelle Hilfsmittel für Heimbewohnende zur Verfügung gestellt werden. Zudem scheinen sich Teilnehmende nicht klar zu dem Gebrauch sexueller Hilfsmittel in der Altenpflege positionieren zu können. Die von der Mehrheit der Befragten ausgewählte Antwortoption „Weiß nicht“ kann hier durchaus als Ausdruck einer echten Meinungslosigkeit verstanden werden, da insgesamt wenig Informationen zum Thema sexuelle Hilfsmittel in Pflegeeinrichtungen vorliegen (vgl. Franzen 2014). Wiederum könnte die hohe Zustimmungstendenz zur Bereitstellung von Hilfsmitteln, um sexuelle Aktivität zu unterstützen, ein Ausdruck sozialer Erwünschtheit in der soziologischen Auslegung des Begriffes sein, dass Teilnehmende in der mutmaßlichen Meinung des Fragenden antworteten (vgl. Krebs 1991). Eine eindeutige Aussage zum Thema sexuelle Hilfsmittel in der Pflege lässt sich daher anhand der vorliegenden Ergebnisse nicht treffen.

\section{Meinungsbild}

Die vorliegende Studie zeigt, dass zu den häufigsten erlebten Reaktionen auf sexuelle Aktivität unter Heimbewohnenden „Ekel“ gehört. Sowohl Altenpflegende $(33,1 \%)$ als auch Familienangehörige $(31,8 \%)$ hatten so reagiert. Auch das „Lächerlich machen“ sexueller Aktivität gehörte mit zu den am häufigsten genannten Reaktionen (s. Abbildung 11 und Abbildung 12). Das von den Studienteilnehmenden beobachtete Verhalten deutet darauf hin, dass Alterssexualität, obwohl als existierend anerkannt, inadäquate Reaktionen erfährt, sowohl auf familiärer als auch auf professioneller Ebene. Die in der vorliegenden Studie erlebten Reaktionen deuten an, dass zumindest teilweise eine negative Grundstimmung zu den Themen Sexualität und Paarbildung unter Heimbewohnenden herrscht. Allerdings 
wurden mögliche Gründe hierfür nicht erfragt. Weiterhin wurden in der vorliegenden Studie im Zusammenhang mit Paarbildung oder sexueller Aktivität bewusst nur negative Reaktionen abgefragt (Items 7.4 - 7.12). Teilnehmende hatten vorher die Möglichkeit abzuschätzen, ob diese Situationen toleriert wurden (Items 7.1 - 7.3). Laut Angaben der Befragten wurde insbesondere die Einstellung der Angehörigen negativ eingeschätzt: Nur 15\% ( $\mathrm{n}=85)$ gaben an, dass Angehörige sexuelle Aktivität unter Heimbewohnenden tolerieren würden. Dabei gehörten Ekel und Streit zu den am häufigsten erlebten Reaktionen. In einer Studie von Steffl (1975) hatte sich gezeigt, dass sich viele der im Pflegeheim Arbeitenden eher nach den Wünschen der Angehörigen richteten als nach den Bedürfnissen der Heimbewohnenden (Steffl 1975, zitiert nach Glass et al. 1986). Die Tendenz, die Sexualität der Heimbewohnenden als sekundär anzusehen, zeigte sich insbesondere in den Leitungspositionen (vgl. Glass et al. 1986). Sexuelle Aktivität unter Bewohnenden zu tolerieren, könnte für Leitungspersonen bspw. zu rechtlichen Problemen führen, weshalb eine Vernachlässigung der Bewohnendensexualität vieles auf administrativer Ebene vereinfachen würde (vgl. Glass et al. 1986). Grund hierfür sei, so vermuteten die Autoren, dass diese zusätzlich ein Verantwortungsgefühl gegenüber anderen Bewohnenden und ihren Angehörigen hegten (vgl. Glass et al. 1986).

In der Pflegeheimsituation bekomme das Thema Sexualität durch Tuscheln und Tratschen einen pubertären Charakter (van der Vight-Klußmann 2014). Durch Restriktionen im Pflegealltag, die bspw. durch Mehrbettzimmer und mangelnde Privatsphäre entstehen, nimmt die sexuelle Aktivität unter Heimbewohnenden ab (van der Vight-Klußmann 2014). Durch mangelnde Privatsphäre wird im institutionalisierten Leben das eigentlich Private nun öffentlich gemacht - so auch das Ausleben von Sexualität (Kleinevers 2004). Doch wenn Heimbewohnende während einer sexuellen Aktivität „erwischt“ werden, dann kommt es zu peinlichen Situationen (van der Vight-Klußmann 2014). Die Autorin Kleinevers (2004) beobachtete, ebenso wie auch durch die vorliegende Studie belegt, unter anderem die Reaktionen Scham, Peinlichkeit, Ekel, Lust und Schuld bei Pflegenden. Diese Gefühle wurden nicht nur in sexuell-konnotierten Situationen empfunden, sondern auch als Reaktion auf pflegerische Handlungen, beispielsweise bei der Intimpflege (Kleinevers 2004). Einen Lösungsansatz zum Umgang mit diesen Gefühlen beschreibt Grond (2001) in der Selbstreflexion. Pflegende sollten sich bewusstwerden, welche Situationen im Pflegealltag Gefühle wie Scham und Ekel auslösen, diese reflektieren und bspw. in Rücksprache mit Kollegen eine standardisierte Lösung im Umgang mit auslösenden Situationen finden (vgl. Grond 2001). 


\section{Meinungsbild: Welche Bedeutung haben sexuell übertragbare Erkrankungen und die ärztliche Versorgung in der Altenpflege?}

Teil der Fragestellung von Q 2 war der Aspekt der ärztlichen Versorgung in der Altenpflege, der in Bezug auf die sexuelle Gesundheit der Bewohnenden beleuchtet werden sollte. Die Erhebungen der vorliegenden Studie deuten darauf hin, dass die Studienteilnehmenden Arztbesuche durch Gynäkologen und Urologen im Altenpflegeheim zwar für sinnvoll halten, aber nur unzureichend von Seiten der Pflege darauf geachtet wird, dass es auch zu Vorsorgeuntersuchungen komme. Laut der Studie zur ärztlichen Versorgung in Pflegeheimen (Sävip) zeigte sich in den 407 befragten Heimen, dass die ärztliche Versorgungssituation ungenügend war (Hallauer et al. 2005). Unter anderem sei es bei einer durchschnittlichen Bewohnerzahl von 77,16 Bewohnenden pro Heim selten zu Besuchen durch Ärzte der Gynäkologie (4,7 Besuche pro Heim pro Jahr) und Urologie (21,2 Besuche pro Heim pro Jahr) gekommen (vgl. Hallauer et al. 2005). Die Autoren befanden, dass insbesondere die gynäkologisch-urologische Versorgung aufgrund der Vielzahl entsprechender Krankheitsbilder (über 72\% der Heimbewohnenden sind Urin-inkontinent) mangelhaft ist (vgl. Hallauer et al. 2005). In Zusammenschau der Ergebnisse beider Studien kann davon ausgegangen werden, dass die ärztliche Versorgung, die unter anderem die sexuelle Gesundheit der Heimbewohnenden fördern würde, insgesamt nur wenig bzw. unzureichende Beachtung im Pflegealltag findet.

Anlehnend an die ärztliche Versorgung in Altenpflegeheimen, werden nun die wesentlichen Ergebnisse zu dem Thema sexuell übertragbare Erkrankungen (STDs) in diesem Abschnitt zusammengefasst. Ziel der vorliegenden Studie war es nicht, die Prävalenzen von STDs zu erheben, sondern $\mathrm{zu}$ untersuchen, inwieweit sexuell übertragbare Erkrankungen im Pflegealltag präsent waren. Dennoch legen die Ergebnisse zu den im Berufsalltag erlebten STDs nahe, dass zum Erhebungszeitpunkt zumindest einige Bewohnende in den teilnehmenden Altenpflegeheimen erkrankt waren. Limitierend sollte berücksichtigt werden, dass von den in der vorliegenden Studie aufgeführten Erkrankungen sexuelle Kontakte nicht den einzigen Übertragungsweg darstellen (vgl. Herold 2016). 70,1\% der befragten Altenpflegenden befand, dass es wichtig wäre, sexuell aktive Heimbewohnende über sexuell übertragbare Erkrankungen aufzuklären. In der Forschungsliteratur wurde von einem Anstieg sexuell übertragbarer Erkrankungen unter älteren Menschen in England und den USA berichtet (vgl. von Simson und Kulasegaram 2012). In einer englischen Studie, die sich mit der sexuellen Aktivität und dem sexuellen Risikoverhalten von über 50-Jährigen befasste, zeigte sich, dass sich 7\% der Studienteilnehmenden Situationen aussetzten, die mit einem 
erhöhten Infektionsrisiko für STDs einhergingen (Gott 2001). Fast alle Befragten gaben an, wenig Informationen zum Thema HIV und STDs erhalten zu haben. Ein Viertel der Teilnehmenden gab an, mehr Informationen zu diesen Themen erhalten zu wollen (Gott 2001). In ihrer Studie befand Gott (2001), dass entsprechendes Personal im Sozial- und Gesundheitswesen über die sexuelle Gesundheit älterer Menschen unterrichtet werden sollte. Zusammenfassend geben die vorliegenden Daten zu erkennen, dass sexuell übertragbare Erkrankungen unter sexuell aktiven Älteren eine Relevanz haben (vgl. von Simson und Kulasegaram 2012) und dass sowohl unter Altenpflegenden als auch unter sexuell aktiven Älteren Aufklärungsbedarf herrscht (vgl. Gott 2001).

\subsection{Q 3: Was ist der aktuelle Wissensstand in der Altenpflege zum Thema Sexualität und sexuelle Gesundheit?}

Der Wissensstand der Studienteilnehmenden zum Thema Sexualität war in der vorliegenden Arbeit von Unsicherheit und fehlendem Fachwissen geprägt. Dies steht im Widerspruch zur subjektiv empfundenen Aufgeklärtheit der Teilnehmenden (s. Abbildung 19). Beispielsweise befanden 8,3\% $(\mathrm{n}=46)$ der Befragten, dass Homosexualität eine Krankheit sei und weitere $13,9 \%(n=77)$ waren sich dessen nicht sicher. Insgesamt wurden Items, die sich mit dem Wissensstand der Teilnehmenden zum Thema Sexualität befassten, häufig mit „Weiß nicht“ beantwortet. Dennoch gilt bei der Interpretation der Ergebnisse zu beachten, dass diese Antwortoption zum einen eine echte Unwissenheit oder auch eine Meinungslosigkeit zum Ausdruck bringen will (vgl. Franzen 2014). Zum anderen bietet sie aber auch die Möglichkeit, dass Befragte bei der Beantwortung den kognitiven Aufwand reduzieren (Franzen 2014). Turner et al. (2014) fanden heraus, dass auch der Wissensstand von Medizinstudenten in Deutschland im Rückstand ist. In einer Fragebogenstudie vorklinischer Ulmer Medizinstudenten des Jahrgangs 1985/1986 hielten 19\% der Befragten Homosexualität für eine psychische Störung und 28\% waren unentschlossen (vgl. Brähler und Böhm 1988). Allerdings gilt es hier zu beachten, dass zum Zeitpunkt dieser Erhebung der Paragraph 175 des Strafgesetzbuches noch in Kraft gesetzt war, wonach Homosexualität unter Männern in der Bundesrepublik Deutschland eine Straftat war (Bundeszentrale für politische Bildung 2014). Erst im März 1994 wurde dieser Paragraph aus dem Strafgesetzbuch gestrichen (Bundeszentrale für politische Bildung 2014).

Die Studie Jugendsexualität 2015 wies Homosexualität ebenfalls als „Tabuthema“ aus (vgl. Bode und Heßling 2015). So hatten nur um die 40\% (Varianz um den Wert 40\%, je nach Geschlecht und Herkunft) der befragten Jugendlichen das Thema Homosexualität im Sexualkundeunterricht behandelt (vgl. Bode und Heßling 2015). Diese Ergebnisse machen 
deutlich, dass auch knapp 30 Jahre später noch die Notwendigkeit der sexuellen Aufklärung besteht, insbesondere mit Schwerpunktsetzung im Bereich der sexuellen Orientierung (vgl. Brähler und Böhm 1988; Turner et al. 2014; Bode und Heßling 2015). Insgesamt wurden sowohl in der vorliegenden Studie als auch in der Studie von Turner et al. (2014) Wissensfragen zu Sexualität sowie Fragen über sexuelle Mythen oft falsch beantwortet. Die Ergebnisse beider Studien lassen den Schluss zu, dass das Thema Sexualität sowohl in der Altenpflegeausbildung als auch im Medizinstudium wenig Beachtung findet (vgl. Turner et al. 2014).

\subsection{Q 4: Wie wird die eigene Sexualität, die Sexualität älterer Menschen und die Sexualität eigener Angehöriger wahrgenommen?}

Im Folgenden wird die Wahrnehmung der eigenen Sexualität und die Sexualität anderer Menschen betrachtet. Studienteilnehmende scheinen insgesamt ihrem subjektiven Empfinden nach ihre eigene Sexualität überwiegend reflektiert zu betrachten. Die Ergebnisse der Items 8.1, 8.7 und 8.8 deuten darauf hin, dass die Mehrheit der Studienteilnehmenden der Meinung war, ein selbstbestimmtes Sexualleben zu führen (s. Abbildung 15). Im Vergleich dazu wurden Items, die die eigene Sexualität negativ und fremdbestimmt darstellten (Items 8.9 - 8.12), von den Befragten als eher nichtzutreffend beantwortet (s. Abbildung 16). Aufgrund der eher polarisierten Antworttendenzen der Teilnehmenden bei den o. g. Items muss an eine Selbstüberschätzung im psychologischen Sinne der sozialen Erwünschtheit gedacht werden (vgl. Krebs 1991). So neigten Teilnehmende an Persönlichkeitsfragebögen dazu, ihre positiven Persönlichkeitsmerkmale im Vergleich zu negativen Merkmalen tendenziell zu überschätzen (Krebs 1991). Diese Verhalten geschieht, um sich selbst in ein positives Licht zu rücken und nach sozialer Anerkennung zu streben. Einen Einfluss sozialer Erwünschtheit auf die Ergebnisse der vorliegenden Studie lassen sich daher zumindest vermuten (vgl. Krebs 1991).

Die Daten der vorliegenden Studie deuten an, dass die Einstellung gegenüber der Sexualität älterer Menschen durch diverse Faktoren beeinflusst wird. Neben dem Ausbildungsstand und dem Alter des Pflegepersonals (s. Kapitel 4.1) scheint auch das Bewohnendenkollektiv eine Rolle zu spielen. So vermuten Bouman et al. (2007), dass Pflegepersonal, welches mit immobilen, multimorbiden Bewohnenden arbeitete, diese eher als asexuell bewertete, als Pflegepersonal, das mit selbstständigen, weniger abhängigen Bewohnenden arbeitete. 
Dennoch scheinen die Teilnehmenden der vorliegenden Studie überwiegend positiv gegenüber der Sexualität älterer Menschen eingestellt zu sein (s. Kapitel 3.4). Doch auch hierbei muss der Aspekt der sozialen Erwünschtheit berücksichtigt werden, wonach Teilnehmende aufgrund der Thematik des Fragebogens das Gefühl haben könnten, dass eine positive Einstellung von ihnen erwartet werden könnte (vgl. Krebs 1991). Nichtsdestotrotz könnte es aufgrund des geringen Alters der Teilnehmenden sein, dass bei mangelnder Erfahrung mit gesellschaftlichen Normen diese auch eher ihre eigene Meinung zum Ausdruck brachten und demnach aufrichtig antworteten (vgl. Krebs 1991). Zudem wird in der Literatur davon ausgegangen, dass eine Reflexion der eigenen Sexualität auch mit einer positiven Einstellung gegenüber der Sexualität älterer Menschen einhergeht, was die Beobachtungen der vorliegenden Studie unterstützen würde (vgl. Grond 2001; de Vries 2011; Kleinevers 2004; Zettl 2000; Michelchen 2008).

Die Ergebnisse der vorliegenden Studie deuteten darauf hin, dass sich die generelle Thematisierung von Sexualität vom thematischen Umgang mit der Sexualität der eigenen Angehörigen unterscheidet (s. Abbildung 14), was auch in einer Studie von Bauer et al. (2013) beobachtet wurde. Obwohl ein Wissenszuwachs bei Pflegenden die Einstellung zum Thema Alterssexualität positiv beeinflussen konnte, konnte diese Tendenz bei der Betrachtung der Sexualität der eigenen Angehörigen eher nicht beobachtet werden (vgl. Bauer et al. 2013). Lewis und Bor (1994) stellten Vermutungen an, wonach es Themenbereiche gäbe, die derart stark von einer emotionalen Komponente geprägt seien, dass diese nicht oder nur schwer durch vermehrtes Wissen zu diesem Thema beeinflusst werden könnten. So schätzen sie, dass die affektive Komponente deutlich schwerer zu ändern sei als die kognitive (vgl. Lewis und Bor 1994). Die Autoren wollen daher Pflegende darauf aufmerksam machen, dass jeder von einer gewissen Voreingenommenheit betroffen ist. Man solle sich diesem Bias bewusstwerden und eine nicht-wertende Umgebung für den Gepflegten schaffen (Lewis und Bor 1994). Um die Sexualität älterer Menschen zu akzeptieren, scheint es wichtig, die eigene Sexualität gut reflektieren zu können. Pflegende, die sich ihrer eigenen sexuellen Gefühle bewusst sind, könnte es leichter fallen, die Sexualität der Gepflegten differenzierter zu betrachten und somit unvoreingenommener ein Umfeld für sexuelle Offenheit zu schaffen (vgl. de Vries 2011; Grond 2011; Lewis und Bor 1994). Dennoch ist davon auszugehen, dass im Pflegealltag zusätzliche Faktoren wie bspw. Zeitund Fachkräftemangel sowie limitierte Räumlichkeiten zusätzlich zur erforderlichen Selbstreflexion die Schaffung eines offenen Umfeldes beschränken können (vgl. Matolycz 2011; Sieren 2012; Grond 2001; Zettl-Wiedner 2011). 


\subsection{Q 5: Wie werden die Themen Sexualität im Elternhaus und Sexualaufklärung behandelt?}

In der vorliegenden Studie wurde die Rolle von Sexualität in der Familie beleuchtet. Die Ergebnisse der Arbeit deuten an, dass wenig Offenheit in der Familie in Bezug auf Sexualität herrscht. Diese scheint insgesamt wenig thematisiert zu werden, wobei insbesondere die Sexualität der Eltern selten angesprochen wurde. Dennoch spielen sowohl Mutter ( $n=230$; $38,5 \%)$ als auch Vater $(n=83 ; 13,9 \%)$ eine Rolle in der Sexualaufklärung der Teilnehmenden. Vor allem für weibliche Studienteilnehmende stellt die Mutter eine größere Aufklärungsquelle dar als der Vater (s. Tabelle 18). Die Bundeszentrale für gesundheitliche Aufklärung (BZgA) zeigte ebenfalls, dass für Mädchen die Mutter die Hauptbezugsperson war, während der Vater eher eine untergeordnete Rolle spielte (Bode und Heßling 2015). Hingegen waren laut BZgA heutzutage beide Eltern bei Jungen gleichbedeutend in der Sexualaufklärung, was in dieser Studie nicht gezeigt werden konnte (vgl. Bode und Heßling 2015). Neben dem Geschlecht der Befragten hatte auch die Herkunft einen Einfluss auf die Rolle des Elternhauses in der Sexualaufklärung (vgl. Bode und Heßling 2015). Während in der Altersgruppe 14 bis 25 Jahre 75\% der weiblichen deutschen Jugendlichen und 65\% der männlichen befragten Deutschen im familiären Rahmen über Partnerschaften und Sexualität gesprochen hatten, waren es im Vergleich dazu bei den männlichen Jugendlichen mit Migrationshintergrund nur 47\% und bei den weiblichen 52\% (vgl. Bode und Heßling 2015). Zudem zeigten die Daten, dass auch innerhalb einer Altersgruppe eine große Varianz herrscht. Es stellte sich heraus, je älter die Teilnehmenden wurden (unabhängig von ihrer Herkunft), desto häufiger wurde mit den Eltern über sexuelle Themen gesprochen (Bode und Heßling 2015).

Die Beobachtungen legen nahe, dass der soziokulturelle und ethnische Hintergrund, der zumindest zu einem gewissen Teil durch die Eltern vorgegeben ist, einen Einfluss auf das Thema Sexualität im Elternhaus hat. Zu beachten ist, dass in der vorliegenden Arbeit weder auf die Religion noch auf den sozio-familiären und sozio-kulturellen Hintergrund der Studienteilnehmenden eingegangen wurde, da im Rahmen der Erhebung dieser Daten gegebenenfalls ein Rückschluss auf einzelne Personen möglich gewesen wäre. Obwohl er in der vorliegenden Studie nicht explizit erfragt wurde, kann der soziokulturelle und ethnische Hintergrund einen Einfluss auf die Einstellung gegenüber Sexualität haben (vgl. Ahrold und Meston 2010). So untersuchten Ahrold und Meston (2010) in ihrer Studie den Einfluss der ethnischen Herkunft auf die Einstellung zu Sexualität bei amerikanischen Collegestudenten mit asiatischem, lateinamerikanischem und europäischem Hintergrund. Im Rahmen ihrer Studie zeigte sich, dass bspw. asiatische Studierende trotz Akkulturation - einem kulturellen 
Anpassungsprozess - konservativer gegenüber den Themen Homosexualität und Gelegenheitssex (Casual Sex) eingestellt waren als andere Befragte. Die Studienergebnisse sprechen also dafür, dass es bei den unterschiedlichen Ethnien gewisse Themen im Bereich Sexualität gibt, die trotz kultureller Integration bzw. Verwestlichung nicht beeinflusst werden können (vgl. Ahrold und Meston 2010). Aus diesen Beobachtungen resultiert, dass der ethnische Hintergrund durchaus einen Einfluss auf die Einstellung zu Sexualität hat. Dennoch wurde diese Perspektive der Thematik Sexualität zur Gewährleistung der Anonymität der Teilnehmenden nicht beleuchtet und kann damit nicht differenziert aufgezeigt werden.

In der vorliegenden Studie hatte sich gezeigt, dass neben dem Elternhaus auch die Schule eine wichtige Rolle in der Sexualaufklärung spielt. So gaben über die Hälfte der Studienteilnehmenden $(n=314 ; 52,5 \%)$ der vorliegenden Studie an, hauptsächlich während ihrer allgemeinen Schulzeit aufgeklärt worden zu sein (s. Tabelle 17). Bei Lewis und Bor (1994) zeigte sich ebenfalls, dass die Institution Schule eine wichtige Rolle in der Sexualaufklärung spielte. Diese Aussage wird auch von den Ergebnissen der Bundeszentrale für gesundheitliche Aufklärung belegt. Diese gab an, dass 93\% der befragten Jugendlichen aus der Bundesrepublik Deutschland in der Schule über Sexualerziehungsthemen unterrichtet wurden und $80 \%$ der Jugendlichen die Schule als Hauptaufklärungsort wahrnehmen würden (vgl. Bode und Heßling 2015). Hiermit kann verdeutlicht werden, dass insbesondere die allgemeine Schulausbildung eine wichtige Rolle in der Sexualaufklärung spielt. Auch zeigten Studien, dass unter anderem eine höhere schulische Bildung und größere Berufserfahrung einen positiven Einfluss auf die Einstellung zu Alterssexualität haben (vgl. Bouman et al. 2007).

Im Zusammenhang mit der vorliegenden Studie muss beachtet werden, dass einige der Teilnehmenden schon vor längerer Zeit oder in anderen Ländern ihren Schulabschluss machten und daher eventuell in gesellschaftlich restriktiveren Perioden die Schule besucht haben könnten. Kulturelle und religiöse Aspekte unterschiedlicher gesellschaftlicher Gruppen sollten insbesondere im Aufklärungsunterricht berücksichtigt werden (vgl. WHORegionalbüro für Europa und BZgA 2011). Auch der Niedersächsische Landtag (2014) gab bekannt, dass Lerninhalte des Sexualkundeunterrichtes an die sexuellen und personellen Identitäten angepasst werden und somit auch zukünftig Themen wie Homo-, Hetero- und Bisexualität einen Einzug in den Unterricht und die Schulbücher finden sollen. Ziel dieser Ergänzung sei es, Diskriminierung entgegenzuwirken und die sexuelle Entwicklung und Diversität der Schüler zu gewährleisten (Niedersächsischer Landtag 2014). Unter 
Berücksichtigung dieser Daten könnten somit zukünftige Generationen von Altenpflegenden einen anderen Blick und eine differenzierte Einstellung zu Sexualität erhalten (vgl. Bode und Heßling 2015; WHO-Regionalbüro für Europa und BZgA 2011; Leitz und Signerski-Krieger 2018). Dennoch muss bei dem Thema Sexualaufklärung berücksichtigt werden, dass - unabhängig von der Sexualerziehung im schulischen Kontext - auch ungesteuerte Prozesse in der sexuellen und geschlechtlichen Entwicklung (sexuelle Sozialisation) in der Peer-Gruppe und im beruflichen Alltag stattfinden (Schmidt 2014).

\subsection{Ausblick}

Die Ergebnisse der vorliegenden Studie erfassten anhand der subjektiven Wahrnehmungen der Befragten examinierten und auszubildenden Altenpflegenden ein Meinungsbild zum Thema sexuelle Gesundheit in der Altenpflege. Es erfolgte eine rein deskriptive Darstellung der Ergebnisse (s. Kapitel 3).

Im Anschluss an diese prospektive Fragebogenstudie wäre ein erneuter Durchlauf zur Überprüfung von Zusammenhangshypothesen und Korrelation von erhobenen Ergebnissen denkbar. Hierfür müsste die statistische Struktur des Fragebogens überarbeitet und angepasst werden. Auch wäre abschließend bspw. eine Längsschnittstudie zu überlegen, um die persönliche Entwicklung bzw. die Einstellung des einzelnen Teilnehmenden bzw. der Ausbildungsjahrgänge im Verlauf der Ausbildung und nach Einstieg in den Berufsalltag zu beobachten.

In Zusammenschau aller Ergebnisse der vorliegenden Studie sowie unter Berücksichtigung der zugrundeliegenden Literatur wären folgende Fragestellungen mit entsprechenden Hypothesen zu formulieren:

\section{Welchen Zusammenhang gibt es zwischen der Wahrnehmung der eigenen Sexualität} und der Sexualität anderer Menschen/der Sexualität von Heimbewohnenden?

Je offener/positiver die Einstellung gegenüber der eigenen Sexualität, desto offener/positiver ist die Wahrnehmung der Sexualität anderer Menschen/ Heimbewohnenden.

\section{Welchen Zusammenhang gibt es zwischen dem Ausbildungsstand der} Teilnehmenden und der Akzeptanz von sexueller Aktivität im Alter/sexueller Aktivität unter Heimbewohnenden?

Je fortgeschrittener der Ausbildungsstand, desto höher ist die Akzeptanz sexueller Aktivität im Alter/sexueller Aktivität unter Heimbewohnenden. 
Welchen Zusammenhang gibt es zwischen dem Alter der Teilnehmenden und der Akzeptanz von sexueller Aktivität im Alter/sexueller Aktivität unter Heimbewohnenden?

Je älter die Teilnehmenden, desto höher ist die Akzeptanz sexueller Aktivität im Alter/sexueller Aktivität unter Heimbewohnenden.

Welchen Einfluss hat größeres Wissen (z. B. durch Fortbildungen/Behandlung des Themas im Unterricht) auf die Einstellung gegenüber sexuell aktiven Heimbewohnenden?

Je mehr Auszubildende/Examinierte über sexuelle Gesundheit in der Altenpflege wissen, desto positiver ist die Einstellung gegenüber sexuell aktiven Heimbewohnenden. 


\section{$5 \quad$ Zusammenfassung}

Die vorliegende Arbeit beschäftigt sich mit der Aktualität der Thematik „Sexuelle Gesundheit in der Altenpflege“. Im Rahmen einer prospektiven Fragebogenstudie wurden Altenpflegende, Auszubildende und Lehrende an Altenpflegeschulen und -heimen in und um Göttingen zu den Themen Sexualität und sexuelle Gesundheit in der Altenpflege befragt. Die Datenerhebung erfolgte in zwei Durchgängen im Zeitraum von Juni bis November 2017. Die Rücklaufquote der Altenpflegenden und Auszubildenden betrug 53,53\% ( $\mathrm{n}=599)$. Bei den Lehrenden ergab sich ein Rücklauf von 35,3\% $(n=30)$.

Ziel der Arbeit war es, eine subjektive Bestandsaufnahme zum Thema sexuelle Gesundheit in der Altenpflege zu erstellen. Dabei wurde die Arbeit in fünf Hauptfragen (Q 1 - Q 5) unterteilt. Es wurden die persönliche Einschätzung zum Stellenwert von Sexualität und sexueller Gesundheit in der Altenpflegeausbildung (Q 1) und im Pflegealltag (Q 2), der Wissensstand zu Sexualität (Q 3), die Wahrnehmung der eigenen Sexualität sowie die Sexualität älterer Menschen und Familienangehörigen (Q 4) erfragt. Von Interesse war ebenfalls die Frage, wie Sexualität im Elternhaus und in der Sexualaufklärung behandelt worden sind (Q 5).

Im Rahmen dieser Fragebogenstudie zeigte sich, dass sexuelle Gesundheit einen diskrepanten Stellenwert in der Altenpflegeausbildung hatte. So hielt die Mehrheit der Befragten das Thema Alterssexualität für ein alltagsrelevantes Ausbildungsfach, obwohl eine Behandlung im Unterricht häufig nicht erfolgt war. Auch die befragten Lehrenden schätzten die Thematik als alltagsrelevant ein und befanden, dass eine Unterrichtung des Themas auf Wunsch der Auszubildenden erfolgen sollte. Im Bereich Fortbildungen zeigte sich, dass Lehrende sich insbesondere didaktische Fähigkeiten zur Vermittlung des Themas Alterssexualität und sexuelle Gesundheit aneignen wollten. Auch Altenpflegende und Auszubildende bekundeten ein Interesse an Fortbildungen, um vor allem den Umgang mit sexuell aktiven Heimbewohnenden erlernen zu können. 57,2\% der befragten Teilnehmenden gaben an, sich gut auf den Umgang mit verbal und körperlich sexuell übergriffigem Verhalten von Heimbewohnenden vorbereitet zu fühlen.

Allgemein scheinen Sexualität und sexuelle Gesundheit im Pflegealltag präsent zu sein. So berichtete fast die Hälfte der Teilnehmenden, bereits sexuelle Aktivität unter Heimbewohnenden erlebt zu haben. Zudem waren sexuell übertragbare Erkrankungen im Pflegealltag zwar gegenwärtig, dennoch wurde eher seltener auf eine ärztliche Anbindung in den Bereichen Urologie und Gynäkologie geachtet. Weiterhin gaben die Befragten an, dass es sowohl von Seiten der Bewohnenden als auch von Pflegenden zu verbalen und körperlichen sexuellen Übergriffen gekommen sei. Die Studienteilnehmenden hatten eingeschätzt, dass 
zu wenig Privatsphäre für Heimbewohnende geboten wird und dass kaum sexuelle Hilfsmittel zur Verfügung gestellt werden. Laut Angaben der Befragten zeigten sich Angehörige am wenigsten tolerant gegenüber sexueller Aktivität und Bildung neuer Partnerschaften unter Heimbewohnenden. In Zusammenschau der Ergebnisse scheint Alterssexualität zumindest teilweise weiterhin ein Tabuthema $\mathrm{zu}$ sein. So deuten die Beobachtungen der Teilnehmenden darauf hin, dass durch fehlende Privatsphäre und Offenheit sexuelle Gesundheit in einer Heimsituation aktuell nicht vollständig gewährleistet werden kann.

Der Wissensstand der Teilnehmenden war von Unwissenheit und Unsicherheit geprägt und stand damit im Gegensatz zur subjektiv empfundenen Aufgeklärtheit. Die Mehrzahl der Befragten gab an, eine überwiegend positive Einstellung zur eigenen Sexualität und zu der Sexualität anderer, auch älterer Menschen zu haben. Besonders jüngere Teilnehmende standen der Sexualität älterer Menschen eher positiv gegenüber. Generell zeigte sich zum Thema Offenheit in der Familie und zur Sexualität der eigenen Angehörigen eine eher restriktive Einstellung. Insbesondere die Sexualität der Eltern wurde zurückhaltend thematisiert. Die Mehrheit der Teilnehmenden gab als Hauptaufklärungsquelle die Institution Schule an.

Zusammenfassend muss berücksichtigt werden, dass die Ergebnisse der vorliegenden Studie bestimmten Einschränkungen unterliegen. So spiegeln die Ergebnisse die subjektive Einschätzung der Teilnehmenden, basierend auf ihren Beobachtungen und Erfahrungen zu sexueller Gesundheit, aber nicht zwangsläufig reale Zustände in der Altenpflege wider. Auch der Einfluss von Antworttendenzen, bspw. der sozialen Erwünschtheit oder dem Satisficing muss berücksichtigt werden, da sie zu einer Verzerrung der Ergebnisse führen können. Weiterhin waren die Auszubildenden in der Stichprobe überrepräsentiert. Daher ist eine Übertragung der Ergebnisse auf die Grundgesamtheit der Altenpflegenden nur eingeschränkt möglich.

Abschließend deuten die Ergebnisse der vorliegenden Studie sowie die einschlägige Literatur darauf hin, dass sexuelle Gesundheit im Altenpflegealltag, sowohl für die Bewohnenden als auch für Altenpflegende, ein aktuelles und relevantes Thema darstellt. Viele Autoren fordern die Reflexion der eigenen Sexualität, um einen guten Umgang mit sexuell aktiven Heimbewohnenden zu finden. Sexuelle Gesundheit scheint gegenwärtig gering in der Altenpflegeausbildung und im Pflegealltag priorisiert zu werden. So könnte der Umgang mit sexueller Gesundheit zukünftig - bspw. anhand eines festgelegten Curriculums oder im Rahmen von Fortbildungen - Einzug in die Aus- und Weiterbildung von Altenpflegenden und Lehrenden finden, um den Wissensstand zu verbessern und Unsicherheiten zu überwinden. 


\section{$6 \quad$ Anhang}

\subsection{Fragebogen Auszubildende/Altenpflegende}

西

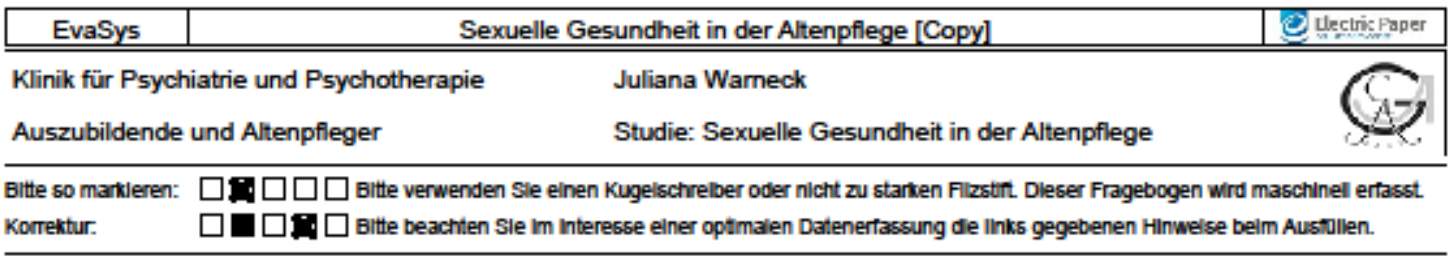

1. Hinweise zum Ausfüllen des Fragebogens

Dieser Fragebogen wird im Rahmen einer Studie zum Thema Sexuelle Gesundheit in der Altenpflege in ausgewählten Regionen Niedersachsens verteilt. Die kommenden Fragen sollen erfassen, welche Situationen Sie erleben und welche Relevanz die Themen sexuelle Gesundheit und Alterssexualität im Arbeitsalltag für Sie haben.

Im Folgenden ist unter dem Begriff Alterssexualitāt nicht nur der Geschlechtsverkehr an sich gemeint, sondern auch jegliche Form von körperlicher Nāhe (Händchenhalten, Küssen, Streicheln) sowie Selbstbefriedigung und das Nutzen von erotischem Bildmaterial.

Aus Grūnden der leichteren Lesbarkeit wird im Text im Allgemeinen auf eine geschlechtsspezifische Differenzierung verzichtet. Entsprechende Begriffe gelten im Sinne der Gleichberechtigung für beide Geschlechter.

Wir bitten Sie, alle Fragen ehrlich und nach bestem Wissen zu beantworten. Diese Umfrage ist keine Prüfung, es gibt keine richtigen oder falschen Antworten. Bitte lassen Sie keine Fragen aus, sondern kreuzen Sie im Zweifel eine Aussage an, die Ihrer Meinung nach am ehesten zutrifft. Sollten Sie ein falsches Kreuz gesetzt haben, dann machen Sie das Kästchen bitte durch Schwärzen unkenntlich und setzten Sie ein neues Kreuz.

Wenn Sie Wünsche oder Anmerkungen haben, kommentieren Sie diese gerne auf der letzten Seite in dem dafur vorgesehenen Textfeld.

Wir versichern Ihnen, dass der Fragebogen anonym behandelt und ausgewertet wird.

Vielen Dank für Ihre Mitarbeit.

\section{Ausbildung}

2.1 Ich fühle mich gut vorbereitet auf den Umgang mit verbal und körperlich sexuell übergriffigem Verhalten von Heimbewohnern.

2.2 Das Thema _Umgang mit Alterssexualität" wurde bei mir in der Ausbildung behandelt.

2.3 Das Thema _Umgang mit Alterssexualität" als Ausbildungsfach halte ich für hilfreich für den Arbeitsalltag.

2.4 Alterssexualität ist nur negativ (z.B. im Sinne $\square$ Ja von übergriffigem Verhalten in der Bewohnerversorgung) thematisiert worden.

$\begin{array}{lllll}\text { Triff zu } \square & \square & \square & \square & \square \text { Triff nicht zu } \\ \text { Triff zu } \square & \square & \square & \square & \square \text { Trifft nicht zu } \\ \text { Trifft zu } \square & \square & \square & \square & \square \text { Trifft nicht zu }\end{array}$

$\square$ Nein

$\square$ Weiß nicht

\section{Konzept Sexualassistenz}

Das Konzept der Sexualassistenz stammt ursprünglich aus der Pflege von Menschen mit Behinderung. Es handelt sich hierbei um Personen, die sexuelle Dienste entgeltlich anbieten. Bei diesen Diensten geht es nicht um Geschlechtsverkehr, sondern um andere Formen der körperlichen Zuwendung. Dazu gehören u.a angeleitetes Masturbieren, Streicheln und andere Zärtlichkeiten. Das Konzept wird im geringen Maße auch in der Altenpflege eingesetzt, um sexuell aktive Ältere in ihren Wünschen zu unterstützen.

3.1 Ist Ihnen das Konzept der Sexualassistenz bekannt?

3.2 Befüworten Sie dieses Konzept?

3.3 Wurde in Ihrem Unterricht dieses Konzept thematisiert?

3.4 Haben Sie die Umsetzung dieses Konzeptes in Ihrem Berufsalltag in einer Pflegeeinrichtung selbst erlebt?

3.5 Wird dieses Konzept in Ihrer Einrichtung umgesetzt?

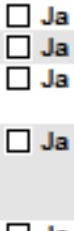

$\square \mathrm{Ja}$ $\square$ Nein

$\square$ Nein

$\square$ Nein

$\square$ Nein

$\square$ Nein $\square$ Weiß nicht

$\square$ Weiß nicht

$\square$ Weiß nicht

$\square$ Weiß nicht

$\square$ Weiß nicht 
EvaSys

Sexuelle Gesundheit in der Altenpflege [Copy]

4. Fortbildungsmöglichkeit am Arbeitsplatz

4.1 Meine Vorgesetzten sorgen für Fortbildungsmöglichkeiten/ Seminare zum Thema Alterssexualität und sexuelle Gesundheit im Altenheim.

$\square$ Ja, und ich habe bereits an derartigen Veranstaltungen teilgenommen.

$\square$ Weiß nicht

4.2 Wünschen Sie sich eine solche Fortbildung?
4.3 Was sollte am ehesten Thema einer solchen Fortbildung sein?

$\square \mathrm{Ja}$, aber ich habe bisher nicht an derartigen Veranstaltungen teilgenommen. $\square$ Nein, derartige Veranstaltungen wurden bisher nicht angeboten. Mehrfachnennungen möglich.

$\square$ Erlernen von Fachwissen über das Thema

$\square$ Erlernen des Umgangs mit sexuell aktiven Heimbewohnern
Nein

$\square$ Weiß nicht

$\square$ Erlemen von Fähigkeiten über sexuelle Vorkommnisse in der Bewohnerversorgung zu reden

$\square$ Eine Fortbildung zum Thema Alterssexualität würde ich nicht besuchen

4.4 Am Arbeitsplatz habe ich eine offizielle Anlaufstelle, um über sexuelle Vorkommnissel Probleme in der Bewohnerversorgung zu reden. Mehrfachnennungen möglich.
$\square$ Ja, mit Betreuer/ Supervisor
$\square$ Ja, mit Betriebsrat
$\square \mathrm{Ja}$, mit Kollegen
$\square$ Ja, mit Vorgesetzten
$\square$ Ja, mit Pflegedienstleitung (PDL)

$\square$ Weiß nicht

4.5 Ich wūnsche mir am Arbeitsplatz eine offizielle Anlaufstelle, um ūber sexuelle Vorkommnisse/ Probleme in der Bewohnerversorgung zu reden.

$\square$ Ja, und ich habe eine.

Ja, ich wünsche mir eine, aber ich habe keine.

$\square$ Nein, ich brauche keine.

\section{Situation in meiner Einrichtung/ meinem Wohnbereich}

5.1 Schätzen Sie, wieviele Ihrer Heimbewohner mit ihren Ehe- oder Lebenspartnem im Altenheim zusammen leben:
$\square 0-25 \%$
$\square 26-50 \%$
$\square$ 51-75\%

$\square 76-100 \%$

5.2 Wie häufig bilden sich unter Heimbewohnern neue Paare?
$\square$ Nie
$\square$ Gelegentlich

$\square$ Regelmäßig

5.3 Wie häufig bilden sich Paare unter Heimbewohnern und Externen?
$\square$ Nie
$\square$ Gelegentlich
$\square$ Regelmāßig

5.4 Haben Sie sexuelle Aktivität unter Heimbewohnern erlebt?
$\square$ Ja
$\square$ Nein

$\square$ Weiß nicht

5.5 „Erwischen" Sie sich selbstbefriedigende Heimbewohner?
$\square$ Nie
$\square$ Gelegentlich
$\square$ Regelmäßig

Im Folgenden beantworten Sie bitte die Hāufigkeit folgender Vorkommnisse Ihrer persönlichen Erfahrung nach

5.6 Verbale sexuelle Ūbergriffe von Heimbewohnern auf Pflegende: $\quad$ z.B. Aufforderungen zu sexuellen Handlungen $\square$ Täglich

$\square$ Wöchentlich

$\square$ Monatlich

$\square$ Seltener $\square$ Nie

5.7 Körperliche sexuelle Übergriffe von Heimbewohnern auf Pflegende: $\quad$ z.B. in den Schritt, Po oder Busen fassen
$\square$ Täglich
$\square$ Wöchentlich
$\square$ Monatlich

$\square$ Seltener

$\square$ Nie

5.8 Verbale sexuelle Übergriffe von Pflegepersonal auf Heimbewohner
$\square$ Täglich
$\square$ Wöchentlich

$\square$ Monatlich

$\square$ Seltener

$\square$ Nie

5.9 Körperliche sexuelle Übergriffe von Pflegepersonal auf Heimbewohner
$\square$ Täglich
$\square$ Wöchentlich
$\square$ Seltener
$\square$ Nie
$\square$ Monatlich

F5534U1846746875P2P10VI

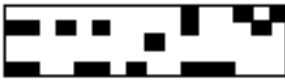


Sexuelle Gesundheit in der Altenpflege [Copy]

5. Situation in meiner Einrichtung/ meinem Wohnbereich

[Fortsetzung]

5.10 Verbale sexuelle Übergriffe unter Heimbewohnern
$\square$ Täglich
$\square$ Wöchentlich
$\square$ Monatlich
$\square$ Seltener
$\square$ Nie

5.11 Körperliche sexuelle Übergriffe unter Heimbewohnern
$\square$ Täglich
$\square$ Wöchentlich
$\square$ Monatlich
$\square$ Seltener
$\square$ Nie

6. Situationen in meinem Berufsallitag

Im Folgenden beantworten Sie bitte die Fragen nach der Häufigkeit der Vorkommnisse

6.1 Auffinden von Dildos:
$\square$ Täglich
$\square$ Wöchentlich
$\square$ Monatlich
$\square$ Seltener
$\square$ Nie

6.2 Auffinden von Gleitgel:
$\square$ Täglich
$\square$ Seltener
$\square$ Wöchentlich
$\square$ Monatlich
$\square$ Nie

6.3 Auffinden von Penisringen:
$\square$ Täglich
$\square$ Seltener
$\square$ Wöchentlich
$\square$ Nie
$\square$ Monatlich

6.4 Auffinden von Potenzmitteln (z.B. Viagra):
$\square$ Täglich
$\square$ Wöchentlich
$\square$ Nie
$\square$ Monatlich

$\square$ Seltener

von Vibratoren
$\square$ Wöchentlich
$\square$ Nie
$\square$ Täglich
$\square$ Monatlich

6 Auffinden von Videos/ Filmen mit pornografischen Inhalten:
$\square$ Seltener
$\square$ Nie

6.7 Auffinden von Zeitschriften mit pornografischen Inhalten:
$\square$ Täglich
$\square$ Wöchentlich
$\square$ Nie
$\square$ Seltener
Monatlich

6.8 Die zuvor beschriebenen Situationen (Selbstbefriedigung. Auffinden von erotischen Zeitschriften, sexuelle Belästigung....) kommen eher vor bei:
$\square$ Mehrheitlich Frauen
$\square$ Mehrheitlich Männern
$\square$ Ich habe keine der hier beschriebenen Situationen
$\square$ Weiß nicht beobachtet

$\square$ Monatlich

9 Werden oben genannte oder andere sexuelle Hilfsmittel von der Einrichtung zur Verfügung gestellt? Mehrfachnennungen möglich.
$\square$ Dildos
$\square$ Potenzmittel (z.B. Viagra)
$\square$ Gleitgel
$\square$ vibratoren
$\square$ Zeitschriften mit pornografischen
$\square$ Nein Inhalten
$\square$ Sonstige

$\square$ Penisringe

$\square$ Videos/Filme mit pornografischen

6.10 Bereitstellung anderer als der hier genannten sexuellen Hilfsmittel in Ihrer Einrichtung:

6.11 Finden Sie die Bereitstellung sexueller Hilfsmittel von der Einrichtung sinnvoll? Mehrfachnennungen möglich.
$\square$ Ja, Dildos
$\square$ Ja, Potenzmittel (z.B. Viagra)
$\square$ Ja, Gleitgel
$\square$ Ja, Vibratoren
$\square$ Ja, Zeitschriften mit
$\square$ Nein
$\square$ Ja, Penisringe
$\square \mathrm{Ja}$, Videos/ Filme mit pornografischen Inhalten
$\square$ Weiß nicht pomografischen Inhalten

$\square$ Ja, sonstige 
EvaSys

Sexuelle Gesundheit in der Altenpflege [Copy]

\section{Situationen in meinem Berufsalltag [Fortsetzung]}

6.12 Die folgenden Hilfsmittel sollten neben den hier genannten sexuellen Hilfsmittel in meiner Einrichtung zur Verfügung gestellt werden:

6.13 Mehrfachnennungen möglich. Die Bereitstellung von sexuellen Hilfsmitteln:

$\square$ Halte ich für sinnvoll, weil Bewohner $\square$ Halte ich für sinnvoll, weil es im selber keine beschaffen kōnnen Alter bei sexuellen Handlungen zu körperlichen Funktionseinschrānkungen kommt

$\square$ Halte ich für sinnvoll, aber denke, dass es zu teuer ist

$\square$ Halte ich nicht für sinnvoll, da ältere Menschen nicht mehr sexuell aktiv sind

$\square$ Halte ich für sinnvoll, weil viele ältere Menschen noch sexuell aktiv sein wollen

$\square$ Halte ich nicht für sinnvoll, da das Ausleben von Sexualität im $\square$ Weiß nicht

$\square$ Halte ich nicht für sinnvoll, da ältere Menschen trotz Hilfsmittel nicht sexuell aktiv sein können Alter abstoßend ist

6.14 Können die Bewohner ihre Sexualität ungestört ausleben? Mehrfachnennungen möglich.

$\square \mathrm{Ja}$, es gibt speziell dafür $\square \mathrm{Ja}$, Bewohner können thre vorgesehene Rāumlichkeiten und Zimmer abschließen Rückzugsorte für Paare

$\square$ Ja, es gibt "Bitte-nicht-stören" Schilder für die Türen der Bewohner

\section{$\square$ Nein}

$\square \mathrm{Ja}$, es gibt die Möglichkeit, dass Paare in einem Doppelzimmer wohnen

$\square$ WeiB nicht

6.15 Klopfen Sie im Arbeitsalltag an, bevor Sie ein Zimmer betreten? Ausgenommen sind hier Notfallsituationen und Bewohner, die nicht mehr adäquat reagieren können.

6.16 Warten Sie nach dem Anklopfen auch auf ein $\quad \square$ Ja $\quad \square$ Nein $\quad \square$ Trifft nicht zu, ich „Herein" des Zimmerbewohners?

$\square \mathrm{Ja}$

7.1 Wird Ihrer Meinung nach sexuelle Aktivität unter Heimbewohnern von anderen Heimbewohnern toleriert?

7.2 Wird threr Meinung nach sexuelle Aktivität unter Heimbewohnem vom Pflegepersonal toleriert ?

7.3 Wird threr Meinung nach sexuelle Aktivität unter Heimbewohnem von Angehörigen toleriert?

$\square$ Ja $\quad \square$ Nein $\quad \square$ Weiß nicht
klopfe nicht an

7.4 Häufigste Reaktionen der anderen Heimbewohner bei Ablehnung: Mehrfachnennungen möglich.
$\square$ Eifersucht
$\square$ Ekel
$\square$ Es lächerlich machen
$\square$ Frust
$\square$ Enttäuschung

$\square \mathrm{Ja}$

$\square$ Nein

$\square$ Weiß nicht

$\square$ Wut

7.5 Häufigste Reaktionen des Pflegepersonals bei Ablehnung: Mehrfachnennungen möglich.
$\square$ Eifersucht
$\square$ Ekel
$\square$ Es lächerlich machen
$\square$ Frust
Enttäuschung

$\square$ Wut

7.6 Häufigste Reaktionen der Familienangehörigen bei Ablehnung: Mehrfachnennungen möglich.
$\square$ Eifersucht
$\square$ Ekel
$\square$ Es lächerlich machen
$\square$ Frust
$\square$ Enttäuschung

$\square$ Wut 
EvaSys

Sexuelle Gesundheit in der Altenpflege [Copy]

7. Meinungsbild [Fortsetzung]

7.7 Wird Ihrer Meinung nach die Neubildung von Partnerschaften_unter Heimbewohnern von

$\square \mathrm{Ja}$

$\square$ Nein

$\square$ Weiß nicht anderen Heimbewohnern toleriert?

7.8 Wird Ihrer Meinung nach die Neubildung von Partnerschaften unter Heimbewohnern vom

$\square \mathrm{Ja}$

$\square$ Nein

$\square$ Weiß nicht Pflegepersonal toleriert?

7.9 Wird Ihrer Meinung nach die Neubildung von Partnerschaften unter Heimbewohnern von

$\square \mathrm{Ja}$

$\square$ Nein

$\square$ Weiß nicht Familienangehörigen toleriert?

7.10 Häufigste Reaktionen der anderen Heimbewohner bei Ablehnung: Mehrfachnennungen möglich.
$\square$ Eifersucht
$\square$ Ekel
$\square$ Frust
$\square$ Enttäuschung
$\square$ Es lächerlich machen
$\square$ Streit

7.11 Häufigste Reaktionen des Pflegepersonals bei Ablehnung: Mehrfachnennungen möglich.
$\square$ Eifersucht
$\square$ Ekel
Enttäuschung
$\square$ Es lächerlich machen
$\square$ Frust
Streit

$\square$ Wut

7.12 Häufigste Reaktionen der Familienangehörigen bei Ablehnung:

Mehrfachnennungen möglich.
$\square$ Eifersucht
$\square$ Ekel
Enttäuschung
$\square$ Es lächerlich machen
$\square$ Frust
$\square$ Streit

$\square$ Wut

7.13 Gönnen Sie es alten Menschen ihre Sexualität aktiv auszuleben?

7.14 Wieviel meinen Sie, wissen Altenpfleger im Durchschnitt über sexuell übertragbare Krankheiten?

7.15 Wieviel meinen Sie, wissen die Bewohner des Altenheims im Durchschnitt über sexuell übertragbare Krankheiten?

$\square$ Weiß nicht Gar nichts $\square \quad \square \quad \square \quad \square \quad \square$ Viel Gar nichts

$\square \quad \square \quad \square \quad \square$ Vie

7.16 Meinen Sie, es ist wichtig sexuell aktive Heimbewohner über sexuell übertragbare

$\square$ Ja Krankheiten aufzuklären?

7.17 Wird von Seiten der Pflege darauf geachtet,

$\square \mathrm{Ja}$ dass es zu Vorsorgeuntersuchungen von Ärzten der Gynäkologie bzw. Urologie kommt?

7.18 Wären solche Arztbesuche lhrer Meinung nach sinnvoll?

$\square \mathrm{Ja}$

$\square$ Nein

$\square$ Nein

$\square$ Nein

$\square$ Weiß nicht

19 Mit welchen der folgenden sexuell übertragbaren Erkrankungen hatten Sie in Ihrem Berufsalltag in der Altenpflege schon zu tun? Mehrfachnennungen möglich.

$\square$ Chlamydien

$\square$ Herpes genitales (HSV)

$\square$ Pilzinfektionen, z.B. Scheidenpilze

$\square$ Gonorrhoe (Tripper)

$\square$ Human Immunodeficiency Virus (HIV)

$\square$ Treponema pallidum (Syphilis) $\square$ Hepatitis B und C

$\square$ Humanes Papilloma Virus (HPVFeigwarzen)

\begin{tabular}{|c|c|c|c|c|}
\hline 7.20 & $\begin{array}{l}\text { Können Frauen auch nach den Wechseljahren } \\
\text { noch Orgasmen haben? }\end{array}$ & $\begin{array}{l}\square \text { Ja, alle Frauen } \\
\square \text { Nein, keine Frau }\end{array}$ & $\begin{array}{l}\square \text { Ja, viele Frauen } \\
\square \text { Weiß nicht }\end{array}$ & $\square$ Ja, wenige Frauen \\
\hline 7.21 & $\begin{array}{l}\text { Können Frauen aus biologischen Gründen } \\
\text { sexuelle Abstinenz besser ertragen als Männer? }\end{array}$ & $\square \mathrm{Ja}$ & $\square$ Nein & $\square$ Weiß nicht \\
\hline 7.22 & Ist Homosexualitāt eine Krankheit? & $\square \mathrm{Ja}$ & $\square$ Nein & $\square$ Weiß nicht \\
\hline 7.23 & $\begin{array}{l}\text { Ist der Mann aus biologischen Gründen sexuell } \\
\text { aktiver als die Frau? }\end{array}$ & $\square \mathrm{Ja}$ & $\square$ Nein & $\square$ Weiß nicht \\
\hline 7.24 & Ist häufiges Masturbieren psychisch schädlich? & $\square \mathrm{Ja}$ & $\square$ Nein & $\square$ Weiß nicht \\
\hline 534018 & 46746875PSPLOVD & & & 27.072017 , Sette $5 / 7$ \\
\hline
\end{tabular}


EvaSys

Sexuelle Gesundheit in der Altenpflege [Copy]

\section{Fragen zur eigenen Sexualität}

8.1 Ich bin mir meiner sexuellen Gefühle sehr bewusst

2 Ich sehe es als positiv an, wenn ältere Personen ein (erfültites) Sexualleben haben.

8.3 Ich kann meine Eltern als sexuell aktiv akzeptieren.

8.4 Ich kann meine Großeltern als sexuell aktiv akzeptieren.

8.5 Das aktive Ausleben von Sexualität im Alter ist etwas Natürliches.

$\begin{array}{lcc}\text { Triff zu } & \square \quad \square \quad \square \quad \square \text { Trifft nicht zu } \\ \square \text { Ja } & \square \text { Nein } & \square \text { Weiß nicht } \\ \square \text { Ja } & \square \text { Nein } & \square \text { Weiß nicht } \\ \square \text { Ja } & \square \text { Nein } & \square \text { Weiß nicht } \\ \square \text { Ja } & \square \text { Nein } & \square \text { Weiß nicht }\end{array}$

8.6 Ich kann offen über Sex reden. Mehrfachnennungen möglich.

$\square \mathrm{Ja}$, mit meinen Eltern $\quad \square \mathrm{Ja}$, mit meinen Freunden $\square$ Ja, mit meinem Partner $\square$ Nein

8.7 Für meine eigene Sexualität bin ich zum Großteil selbst verantwortlich

8.8 Ich denke, dass ich zukünftig ein gesundes und positives Sexualleben haben werde.

8.9 Falls etwas in meinem Sexualleben schiefgeht, würde ich mir selbst die Schuld dafur geben.

8.10 Mir ist es wichtig. wie Mitmenschen mein Sexualverhalten und meine sexuelle Einstellung bewerten.

8.11 Mein sexuelles Verhalten wird hauptsächlich durch Mitmenschen bestimmt (z.B. durch meinen Partner, meine Freunde, meine Familie...).

8.12 Mir fällt schnell auf, wie Mitmenschen die sexuellen Aspekte meines Lebens bewerten.

8.13 Die Beantwortung vorangegangener und folgender Fragen basierten auf. $\square$ Einer aktuellen sexuellen Beziehung $\square$ Einer vergangenen sexuellen Beziehung $\square \mathrm{Ja}$, mit meinen Geschwistern $\square$ Weiß nicht

\begin{tabular}{|c|c|c|c|c|c|c|}
\hline \multicolumn{7}{|c|}{$\square$ Einer aktuellen sexuellen Be } \\
\hline \multicolumn{7}{|c|}{ 9. Offenheit in der Familie } \\
\hline 9.1 & Wie häufig wurde in meiner Familie über Sexualitāt gesprochen? & Sehr häufig $\square$ & $\square$ & $\square$ & $\square$ & $\begin{array}{l}\square \text { Gar nicht } \\
\text { häufig }\end{array}$ \\
\hline 9.2 & Wie offen wurde in meiner Familie über Sexualitāt gesprochen? & Offen $\square$ & $\square$ & $\square$ & $\square$ & $\begin{array}{l}\square \text { Gar nicht } \\
\text { offen }\end{array}$ \\
\hline 9.3 & $\begin{array}{l}\text { Wie offen haben meine Eltern mit mir über Sexualität } \\
\text { gesprochen? }\end{array}$ & Sehr offen $\square$ & $\square$ & $\square$ & $\square$ & $\begin{array}{l}\square \text { Gar nicht } \\
\text { offen }\end{array}$ \\
\hline 9.4 & $\begin{array}{l}\text { Wie offen haben meine Eltern mit mir über ihre eigene } \\
\text { Sexualität gesprochen? }\end{array}$ & Sehr offen $\square$ & $\square$ & $\square$ & $\square$ & $\begin{array}{l}\square \text { Gar nicht } \\
\text { offen }\end{array}$ \\
\hline 9.5 & Fūr wie aufgeklärt halte ich mich persönlich? & $\begin{array}{l}\text { Sehr } \\
\text { aufgeklärt }\end{array}$ & $\square$ & $\square$ & $\square$ & $\begin{array}{l}\square \text { Gar nicht } \\
\text { aufgeklärt }\end{array}$ \\
\hline \multirow[t]{2}{*}{9.6} & $\begin{array}{l}\text { Von wem bin ich hauptsächlich aufgeklärt worden? } \\
\text { Mehrfachnennungen möglich. }\end{array}$ & & & & & \\
\hline & $\begin{array}{l}\square \text { Freunde } \\
\square \text { Mutter } \\
\square \text { Zeitschriften }\end{array}$ & & \multicolumn{4}{|c|}{$\begin{array}{l}\square \text { Geschwister } \\
\square \text { Schule } \\
\square \text { Gar nicht }\end{array}$} \\
\hline
\end{tabular}

9.7 Über folgendes bin ich von meinen Eltern aufgeklärt worden: Mehrfachnennungen möglich.
$\square$ Geburt
$\square$ Homosexualität
$\square$ Geschlechtskrankheiten
$\square$ Selbstbefriedigung
$\square$ Monatliche Blutung (Periode)
$\square$ Sexuelle Funktionsstörungen

$\square$ Gar nicht 
EvaSys

Sexuelle Gesundheit in der Altenpflege [Copy]

\section{Allgemeiner Teil}

10.1 Alter

10.2 Geschlecht

10.3 Beziehungsstatus

$\square$ Feste Partnerschaft

$\square$ Verheiratet

10.4 Sexuelle Orientierung

$\square$ Asexuell

$\square$ Homosexuell

10.5 Berufsausbildung

$\square$ Abgeschlossen

$\square$ Ausbildung 3 . Jahr

10.6 Berufserfahrung im jetzigen Beruf

10.7 Studienabschluss

10.8 Träger des Altenheims

10.9 Ich arbeite im folgenden Bereich:

Mehrfachnennunen möglich.

$\square$ Ambulante Wohngemeinschaft/ Alten-WGs

$\square$ Stationäre Pflege

$\square$ Stationäre Pflege Demenz erkrankt sind:

Betreutes Wohnen

$\square$ Teilstationäre Pflege

$\square<25$ Jahre

$\square$ 45-55 Jahre

$\square$ Männlich

$\square$ 25-35 Jahre

55-60 Jahre

$\square$ Weiblich

$\square$ Offene Beziehung

$\square$ Verwitwet

$\square$ Single

$\square$ Sonstiges

$\square$ Bisexuell

$\square$ Sonstiges

$\square$ Ausbildung 1. Jahr

$\square$ Keine Ausbildung in der Altenpflege

$\square$ Heterosexuell

$\square<1$ Jahr

$\square$ 5-10 Jahre

$\square \mathrm{Ja}$

$\square$ Kirche

$\square$ Wohlfahrtsverband

$\square$ 1-3 Jahre

$\square$ 10-20 Jahre

$\square$ Nein

$\square$ Öffentlich

$\square$ Sonstiges

$\square$ Mehrgenerationenhaus

$\square$ Sonstiges

$\square 26-50 \%$

口 $0-25 \%$

$\square 76-100 \%$ $\square$ 3-5 Jahre $\square>20$ Jahre

$\square$ Privat $\square$ 35-45 Jahre

$\square>60$ Jahre

$\square$ Sonstiges

\section{Anmerkungen}

11.1 Wünsche und Anregungen sind an dieser Stelle wilkommen. 


\subsection{Fragebogen Lehrende}

\begin{tabular}{l|l|l|}
\hline EvaSys & Sexuelle Gesundheit in der Altenpflege [Copy] & dectri: Paper \\
\hline \hline \\
Klinik für Psychiatrie und Psychotherapie & Juliana Warneck \\
Lehrende & Studie: Sexuelle Gesundheit in der Altenpflege
\end{tabular}

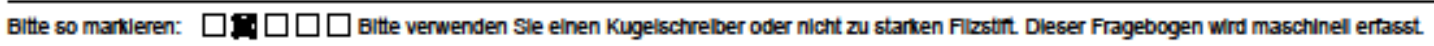
Korrektur. $\quad \square \square \square \square$ Bltte beachten Sle in Interesse elner optmalen Datenertassung de links gegebenen Hinwelse belm Austollen.

\section{Hinweise zum Ausfüllen des Fragebogens}

Dieser Fragebogen wird im Rahmen einer Studie zum Thema Sexuelle Gesundheit in der Altenpflege in ausgewählten Regionen Niedersachsens verteilt. Die kommenden Fragen sollen erfassen, welche Situationen Sie erleben und welche Relevanz die Themen sexuelle Gesundheit und Alterssexualität im Arbeitsalltag für Sie haben.

Im Folgenden ist unter dem Begriff Alterssexualität nicht nur der Geschlechtsverkehr an sich gemeint, sondern auch jegliche Form von körperlicher Nāhe (Händchenhalten, Küssen, Streicheln) sowie Selbstbefriedigung und das Nutzen von erotischem Bildmaterial.

Aus Grūnden der leichteren Lesbarkeit wird im Text im Allgemeinen auf eine geschlechtsspezifische Differenzierung verzichtet. Entsprechende Begriffe gelten im Sinne der Gleichberechtigung für beide Geschlechter.

Wir bitten Sie, alle Fragen ehrlich und nach bestem Wissen zu beantworten. Diese Umfrage ist keine Prüfung, es gibt keine richtigen oder falschen Antworten. Bitte lassen Sie keine Fragen aus, sondern kreuzen Sie im Zweifel eine Aussage an, die Ihrer Meinung nach am ehesten zutrifft. Sollten Sie ein falsches Kreuz gesetzt haben, dann machen Sie das Kästchen bitte durch Schwärzen unkenntlich und setzten Sie ein neues Kreuz.

Wenn Sie Wünsche oder Anmerkungen haben, kommentieren Sie diese gerne auf der letzten Seite in dem dafür vorgesehenen Textfeld.

Wir versichern Ihnen, dass der Fragebogen anonym behandelt und ausgewertet wird.

Vielen Dank für Ihre Mitarbeit.

\section{Ausbildung}

2.1 Wenn Sie eine Ausbildung gemacht haben: Das Thema _Umgang mit Alterssexualität" wurde in meiner Ausbildung behandelt.

2.2 Wenn Sie ein Studium absolviert haben: Das Thema _Umgang mit Alterssexualität" wurde in meinem Studium behandelt.

2.3 Ich fühle mich gut ausgebildet, den Schülern den Umgang mit sexuellem Erleben von Heimbewohnern zu vermitteln.

2.4 Ich fühle mich gut ausgebildet, den Schülern den $\quad$ Triff zu $\square \quad \square \quad \square \quad \square \quad \square$ Trifft nicht zu Umgang mit sexuell übergriffigem Verhalten von Heimbewohnern zu vermitteln.

2.5 Das Thema _Umgang mit Alterssexualität"als Teil $\quad$ Triff zu $\square \quad \square \quad \square \quad \square \quad \square$ Trifft nicht zu eines Studiums halte ich für hilfreich für den Arbeitsalltag. 2.6 Das Thema _Umgang mit Alterssexualität als Ausbildungsinhalt
an Altenpflegeschulen halte ich für hilfreich für den Arbeitsalltag. $\quad$ Trifft zu $\square \quad \square \quad \square \quad \square \quad \square$ Trifft nicht zu

2.7 Alterssexualität ist nur negativ (z.B. im Sinne $\quad \square \mathrm{Ja}$ von übergriffigem Verhalten in der

$\square$ Ja $\square$ Nein

$\square$ Trifft nicht zu Bewohnerversorgung) thematisiert worden 
EvaSys

Sexuelle Gesundheit in der Altenpflege [Copy]

3. Das Thema Sexualität" in der Rolle als Lehrkraft

3.1 Ist das Thema Alterssexualität" Teil des Curriculum an der Altenpflegeschule, an der Sie unterrichten?

3.2 Das Thema Alterssexualität" ist optionaler Teil des Curriculums an der Altenpflegeschule,

$\square \mathrm{Ja}$, verpflichtend

$\square$ Ja, optional

$\square$ Nein

an der Sie unterrichten. Gehen Sie persönlich

$\square \mathrm{Ja}$

$\square$ Nein

$\square$ Trifft nicht zu auf das Thema ein?

3.3 Wie viele Lehrstunden umfasst das Thema Alterssexualität" insgesamt?

3.4 Können Sie sich vorstellen, warum die Vermittlung des Themas von der Schulleitung gewünscht wird? Mehrfachnennungen möglich.

$\square$ Gewünscht, weil es als wichtig für den Arbeitsalltag angesehen wird

$\square$ Gewūnscht, weil das Thema an anderen Schulen unterrichtet wird

$\square$ Gewünscht, da es der Schulleitung aus persönlichen Gründen wichtig ist

$\square$ Gewünscht, da die Schūler über die Thematik unterrichtet werden wollen $\square$ Gewünscht, um einen Wettbewerbsvorteil anderen Schulen gegenüber zu haben

$\square$ Gewünscht aus sonstigen Gründen

3.5 Können Sie sich vorstellen, warum die Vermittlung des Themas von der Schulleitung nicht gewünscht wird? Mehrfachnennungen möglich

$\square$ Ungewünscht, weil es als unwichtig für den Arbeitsalltag angesehen wird

$\square$ Ungewūnscht aus religiösen Gründen

Ungewünscht, weil es zusätzliche Kosten verursacht

$\square$ Ungewünscht, weil das Thema an anderen Schulen unterrichtet wird

3.6 Sollte Ihrer Meinung nach im Unterricht an

$\square \mathrm{Ja}$

$\square$ Nein

$\square$ Ungewünscht aus Schamgefühlen Altenpflegeschulen auf die Sexualität alter Menschen eingegangen werden?

3.7 Haben Sie darüber nachgedacht, die Vermittlung des Themas Alterssexualität in Ihrem Unterricht zu erweitern? Mehrfachnennungen möglich

$\square \mathrm{Ja}$, weil es vorgeschrieben ist

$\square$ Ja, weil es von den Schülern so gewünscht ist $\square$ Ja, weil ich das Thema für wichtig erachte

$\square$ Ja, weil es von meinem Vorgesetzten so gewünscht ist $\square$ Ja, weil es mir Spaß macht, das Thema im Unterricht zu bearbeiten

$\square$ Ja, weil es von dem Träger der Schule so gewünscht ist

3.8 Haben Sie darūber nachgedacht, die Vermittlung des Themas Alterssexualität in lhrem Unterricht auszusparen/ zu verringern Mehrfachnennungen möglich

$\square$ Ja, aus zeitlichen Gründen

$\square$ Ja, aus finanziellen Gründen

$\square$ Ja, weil den Schülem das Thema unangenehm ist

$\square \mathrm{Ja}$, weil das von meinem Vorgesetzten so gewünscht ist

$\square$ Ja, weil mir das Thema unangenehm ist

$\square \mathrm{Ja}$, weil es von dem Träger der Schule so gewünscht ist Alterssexualität zu unterrichten?

3.10 Wenn nicht zutreffend: Würden Sie sich die Unterrichtung des Themas nach entsprechender
Fortbildung zutrauen?

$\square \mathrm{Ja}$

$\square$ Nein

$\square$ Weiß nicht

3.11 Meine Vorgesetzten sorgen für Weiterbildungsmöglichkeiten/ Seminare zum Thema Alterssexualität und sexuelle Gesundheit im Altenheim.

$\square$ Ja, und ich habe bereits an derartigen Veranstaltungen teilgenommen

$\square$ Ja, aber ich habe bisher nicht an derartigen Veranstaltungen teilgenommen $\square$ Nein, derartige Veranstaltungen wurden bisher nicht angeboten

$\square$ Weiß nicht

3.12 Was sollte am ehesten Thema einer solchen Fortbildung sein? Mehrfachnennungen möglich

$\square$ Erlernen von Fachwissen über das Thema

$\square$ Erlemen von Fähigkeiten um über das Thema zu reden $\square$ Erlemen von didaktischen Fähigkeiten zur Vermittlung des Themas

3.13 Wünschen Sie sich eine solche Fortbildung? $\square \mathrm{Ja}$ $\square$ Nein $\square$ Weiß nicht

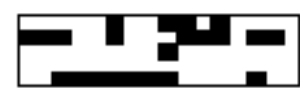


\begin{tabular}{l|l} 
EvaSys & Sexuelle Gesundheit in der Altenpflege [Copy]
\end{tabular}

\section{Erfahrungen und Vorstellungen zum Thema „Sexualität“ aus der Sicht der Pflegekraft}

4.1 Haben Sie Berufserfahrung im Kontext der Altenpflege?
$\square \mathrm{Ja}$, in der Vergangenheit
$\square$ Ja, aktuell
$\square$ Nein, keine Berufserfahrung

Falls Sie keine Berufserfahrung in der Altenpflege haben, fahren Sie bitte mit Teil 5 fort!

4.2 Am Arbeitsplatz habe/ hatte ich eine offizielle Anlaufstelle, um über sexuelle Vorkommnisse/ Probleme in der Bewohnerversorgung zu reden.

Mehrfachnennungen möglich.
$\square$ Ja, mit Betreuer/ Supervisor
$\square$ Ja, mit Betriebsrat
$\square \mathrm{Ja}$, mit Kollegen
$\square$ Ja, mit Vorgesetzten
$\square$ Ja, mit Pflegedienstleitung (PDL)
$\square$ Nein

$\square$ Weiß nicht

4.3 Ich wūnsche/ wūnschte mir am Arbeitsplatz eine offizielle Anlaufstelle, um über sexuelle Vorkommnisse/ Probleme in der Bewohnerversorgung zu reden.
$\square$ Ja, und ich habe eine.
$\square \mathrm{Ja}$, ich wünsche mir eine, aber
$\square$ Nein, ich brauche keine. ich habe keine.

4.4 Schätzen Sie, wieviele Ihrer Heimbewohner mit ihren Ehe- oder Lebenspartnem im Altenheim zusammen leben:
$\square 0-25 \%$
$\square 26-50 \%$
$\square$ 51-75\%

76-100\%

4.5 Wie häufig bilden sich unter Heimbewohnern neue Paare?
$\square$ Nie
$\square$ Gelegentlich
$\square$ Regelmäßig
4.6 Wie häufig bilden sich Paare unter Heimbewohnern und Externen?
$\square$ Nie
$\square$ Gelegentlich
$\square$ Regelmäßig

4.7 Haben Sie sexuelle Aktivität unter Heimbewohnern erlebt?
$\square \mathrm{Ja}$
$\square$ Nein
$\square$ WeiB nicht

4.8 „Erwischen" Sie sich selbstbefriedigende Heimbewohner?
$\square$ Nie
$\square$ Gelegentlich
$\square$ Regelmäßig

Im Folgenden beantworten Sie bitte die Hāufigkeit folgender Vorkommnisse Ihrer persönlichen Erfahrung nach

4.9 Verbale sexuelle Übergriffe von Heimbewohnern auf Pflegende: $\quad$ z.B. Aufforderungen zu sexuellen Handlungen
$\square$ Täglich
$\square$ Wöchentlich
Monatlich

$\square$ Seltener

$\square$ Nie

4.10 Körperliche sexuelle Übergriffe von Heimbewohnern auf Pflegende: $\quad$ z.B. in den Schritt, Po oder Busen fassen
$\square$ Täglich
$\square$ Wöchentlich
$\square$ Monatlich
$\square$ Seltener
$\square$ Nie

4.11 Verbale sexuelle Übergriffe von Pflegepersonal auf Heimbewohner
$\square$ Täglich
$\square$ Wöchentlich
$\square$ Monatlich

$\square$ Seltener

$\square$ Nie

4.12 Körperliche sexuelle Übergriffe von Pflegepersonal auf Heimbewohner
$\square$ Täglich
$\square$ Wöchentlich
$\square$ Monatlich
$\square$ Seltener
$\square$ Nie

4.13 Verbale sexuelle Übergriffe unter Heimbewohnern
$\square$ Täglich
$\square$ Wöchentlich
$\square$ Monatlich
$\square$ Seltener
$\square$ Nie

4.14 Körperliche sexuelle Übergriffe unter Heimbewohnern

$\square$ Täglich

$\square$ Wöchentlich

$\square$ Monatlich

$\square$ Seltener

$\square$ Nie 
\begin{tabular}{l|l} 
EvaSys & Sexuelle Gesundheit in der Altenpflege [Copy]
\end{tabular}

\section{Erfahrungen und Vorstellungen zum Thema „Sexualität" aus der Sicht der Pflegekraft [Fortsetzung]}

Im Folgenden beantworten Sie bitte die Fragen nach der Häufigkeit der Vorkommnisse

4.15 Auffinden von Dildos:
$\square$ Täglich
$\square$ Wöchentlich
$\square$ Monatlich
$\square$ Seltener
$\square$ Nie

4.16 Auffinden von Gleitgel:
$\square$ Täglich
$\square$ Wöchentlich
$\square$ Monatlich
$\square$ Seltener
$\square$ Nie

4.17 Auffinden von Penisringen:
$\square$ Täglich
$\square$ Wöchentlich
$\square$ Seltene
$\square$ Nie
$\square$ Monatlich

4.18 Auffinden von Potenzmitteln (z.B. Viagra):
$\square$ Täglich
$\square$ Wöchentlich
$\square$ Nie
$\square$ Seltene
$\square$ Wöchentlich
$\square$ Nie
$\square$ Täglich
$\square$ Seltener

$\square$ Täglich $\square$ Wöchentlich

$\square$ Seltener $\square$ Nie

4.21 Werden oben genannte oder andere sexuelle Hilfsmittel von der Einrichtung zur Verfügung gestellt? Mehrfachnennungen möglich.
$\square$ Dildos
$\square$ Gleitgel
$\square$ Potenzmittel (z.B. Viagra)
$\square$ Vibratoren
$\square$ Penisringe
$\square$ Videos/Filme mit pornografischen
$\square$ Zeitschriften mit pornografischen
$\square$ Nein Inhalten Inhalten
$\square$ Weiß nicht

$\square$ Sonstige

4.22 Bereitstellung anderer als der hier genannten sexuellen Hilfsmittel in Ihrer Einrichtung:

4.23 Die zuvor beschriebenen Situationen (Selbstbefriedigung. Auffinden von erotischen Zeitschriften, sexuelle Belästigung....) kommen eher vor bei:
$\square$ Mehrheitlich Frauen
$\square$ Mehrheitlich Männern
Ich habe keine der hier
$\square$ Fraven und Männer gleichermaßen
$\square$ Weiß nicht beschriebe

4.24 Können die Bewohner ihre Sexualität ungestört ausleben? Mehrfachnennungen möglich.
$\square$ Ja, es gibt speziell dafür vorgesehene Rāumlichkeiten und
Rückzugsorte für Paare
$\square$ Ja, Bewohner können thre Zimmer abschließen
$\square \mathrm{Ja}$, es gibt die Möglichkeit, dass
Nein Paare in einem Doppelzimmer wohnen
$\square$ Ja, es gibt "Bitte-nicht-stören"
$\square$ WeiB nicht Sa, es gibt "Bitte-nicht-stören"
Schilder für die Türen der Bewohner

4.25 Klopfen Sie im Arbeitsalltag an, bevor Sie ein Zimmer betreten? Ausgenommen sind hier Zimmer betreten? Ausgenommen sind hier Notfallsituationen und Bewohner,
mehr adäquat reagieren können.

4.26 Warten Sie nach dem Anklopfen auch auf ein „Herein"des Zimmerbewohners?

$\square \mathrm{Ja}$

$\square \mathrm{Ja}$

$\square$ Nein 
\begin{tabular}{l|c} 
EvaSys & Sexuelle Gesundheit in der Altenpflege [Copy]
\end{tabular}

\section{Erfahrungen und Vorstellungen zum Thema „Sexualität" aus der Sicht der Pflegekraft [Fortsetzung]}
4.27 Wird Ihrer Meinung nach sexuelle Aktivität
$\square \mathrm{Ja}$
$\square$ Nein
$\square$ Weiß nicht unter Heimbewohnern von anderen Heimbewohnern toleriert?
4.28 Wird Ihrer Meinung nach sexuelleAktivität unter Heimbewohnem vom Pflegepersonal toleriert?

$\begin{array}{lll}\square \text { Ja } & \square \text { Nein } & \square \text { Weiß nicht } \\ \square \text { Ja } & \square \text { Nein } & \square \text { Weiß nicht }\end{array}$
4.29 Wird Ihrer Meinung nach sexuelleAktivität unter Heimbewohnern von Angehörigen toleriert?

4.30 Häufigste Reaktionen der anderen Heimbewohner bei Ablehnung: Mehrfachnennungen möglich.
$\square$ Eifersucht
$\square$ Ekel
$\square$ Enttäuschung
$\square$ Es lächerlich machen
$\square$ Frust
Streit

$\square$ Wut

4.31 Häufigste Reaktionen des Pflegepersonals bei Ablehnung:

Mehrfachnennungen möglich.
$\square$ Eifersucht
$\square$ Ekel
$\square$ Es lächerlich machen
$\square$ Frust
$\square$ Enttäuschung

$\square$ Wut

4.32 Häufigste Reaktionen der Familienangehörigen bei Ablehnung: Mehrfachnennungen möglich.
$\square$ Eifersucht
$\square$ Ekel
$\square$ Es lächerlich machen
$\square$ Frust
$\square$ Enttäuschung
$\square$ Wut

$\begin{array}{lccc}\text { 4.33 Wird Ihrer Meinung nach die Neubildung von } & \square \text { Ja } & \square \text { Nein } & \square \text { Weiß nicht } \\ \text { Partnerschaften unter Heimbewohnern von } & & \\ \text { anderen Heimbewohnern toleriert ? } & & \square \text { Nein } & \square \text { Weiß nicht } \\ \text { 4.34 Wird Ihrer Meinung nach die Neubildung von } & \square \text { Ja } & \\ \text { Partnerschaften unter Heimbewohnern vom } & & \square \text { Nein } & \square \text { Weiß nicht } \\ \text { Pflegepersonal toleriert? } & & \end{array}$

4.36 Häufigste Reaktionen der anderen Heimbewohner bei Ablehnung: Mehrfachnennungen möglich.
$\square$ Eifersucht
$\square$ Ekel
$\square$ Enttäuschung
$\square$ Es lächerlich machen
$\square$ Frust
$\square$ Streit

$\square$ Wut

4.37 Häufigste Reaktionen des Pflegepersonals bei Ablehnung:

Mehrfachnennungen möglich.
$\square$ Eifersucht
$\square$ Ekel
$\square$ Es lächerlich machen
$\square$ Frust
$\square$ Enttäuschung

$\square$ Wut

4.38 Häufigste Reaktionen der Familienangehörigen bei Ablehnung:

Mehrfachnennungen möglich.
$\square$ Eifersucht
$\square$ Ekel
$\square$ Es lächerlich machen
$\square$ Frust
$\square$ Enttäuschung

$\square$ Wut 
EvaSys

Sexuelle Gesundheit in der Altenpflege [Copy]

\section{Konzept Sexualassistenz}

Das Konzept der Sexualassistenz stammt ursprünglich aus der Pflege von Menschen mit

Behinderung. Es handelt sich hierbei um Personen, die sexuelle Dienste entgeltlich anbieten. Bei diesen Diensten geht es nicht um Geschlechtsverkehr, sondern um andere Formen der körperlichen Zuwendung. Dazu gehören u.a angeleitetes Masturbieren, Streicheln und andere Zärtlichkeiten. Das Konzept wird im geringen Maße auch in der Altenpflege eingesetzt, um sexuell aktive Ältere in ihren Wünschen zu unterstützen.

5.1 Ist Ihnen das Konzept der Sexualassistenz bekannt?

5.2 Befüworten Sie dieses Konzept?

5.3 Wurde in Ihrem Unterricht, den Sie unterrichten, dieses Konzept thematisiert?

5.4 Wurde in Ihrem Unterricht während Thre Ausbildungl Ihres Studiums dieses Konzept thematisiert?

5.5 Haben Sie die Umsetzung dieses Konzeptes in lhrem Berufsalltag in einer Pflegeeinrichtung selbst erlebt?
$\square$ Ja
$\square$ Nein
$\square$ Weiß nicht
Trifft nicht zu (keine

Berufserfahrung in der Altenpflege)

5.6 Wird dieses Konzept in lhrer Einrichtung umgesetzt?
$\square \mathrm{Ja}$
$\square$ Nein
$\square$ Weiß nicht
$\square$ Trifft nicht zu (keine
Berufserfahrung in der Altenpflege)

\section{Fragen zur eigenen Sexualität}

6.1 Ich bin mir meiner sexuellen Gefühle sehr bewusst.

6.2 Ich sehe es als positiv an, wenn ältere Personen ein (erfülites) Sexualleben haben.

$\begin{array}{lll}\square \mathrm{Ja} & \square \text { Nein } & \square \text { Weiß nicht } \\ \square \mathrm{Ja} & \square \text { Nein } & \square \text { Weiß nicht } \\ \square \mathrm{Ja} & \square \text { Nein } & \square \text { Weiß nicht } \\ \square \mathrm{Ja} & \square \text { Nein } & \square \text { Weiß nicht }\end{array}$

6.3 Ich kann meine Eltern als sexuell aktiv akzeptieren.

6.4 Ich kann meine Großeltern als sexuell aktiv akzeptieren.

6.5 Das aktive Ausleben von Sexualitāt im Alter ist etwas Natürliches.

$\begin{array}{lcc} & \text { Trifft zu } \square \quad \square \quad \square \quad \square \quad \square \text { Trifft nicht zu } \\ \square \text { Ja } & \square \text { Nein } & \square \text { Weiß nicht } \\ \square \text { Ja } & \square \text { Nein } & \square \text { Weiß nicht } \\ \square \text { Ja } & \square \text { Nein } & \square \text { Weiß nicht } \\ \square \text { Ja } & \square \text { Nein } & \square \text { Weiß nicht }\end{array}$

6.6 Ich kann offen über Sex reden Mehrfachnennungen möglich.
$\square \mathrm{Ja}$, mit meinen Eltern
$\square \mathrm{Ja}$, mit meinen Freunden
$\square$ Ja, mit meinem Partner
$\square$ Ja, mit meinen Geschwistern
$\square$ Weiß nicht

$\square$ Nein

6.7 Für meine eigene Sexualität bin ich zum Großteil selbst verantwortlich.

6.8 Ich denke, dass ich zukūnftig ein gesundes und positives $\quad$ Trifft zu $\square \quad \square \quad \square \quad \square \quad \square$ Trifft nicht zu Sexualleben haben werde.

6.9 Falls etwas in meinem Sexualleben schiefgeht, würde ich mir selbst die Schuld dafur geben.

6.10 Mir ist es wichtig, wie Mitmenschen mein Sexualverhalten und meine sexuelle Einstellung bewerten.

6.11 Mein sexuelles Verhalten wird hauptsächlich durch Mitmenschen bestimmt (z.B. durch meinen Partner, meine Freunde, meine Familie...).

6.12 Mir fällt schnell auf, wie Mitmenschen die sexuellen Triff zu Aspekte meines Lebens bewerten.

6.13 Die Beantwortung vorangegangener und folgender Fragen basierten auf $\square$ Einer aktuellen sexuellen Beziehung $\square$ Einer vergangenen sexuellen Beziehung

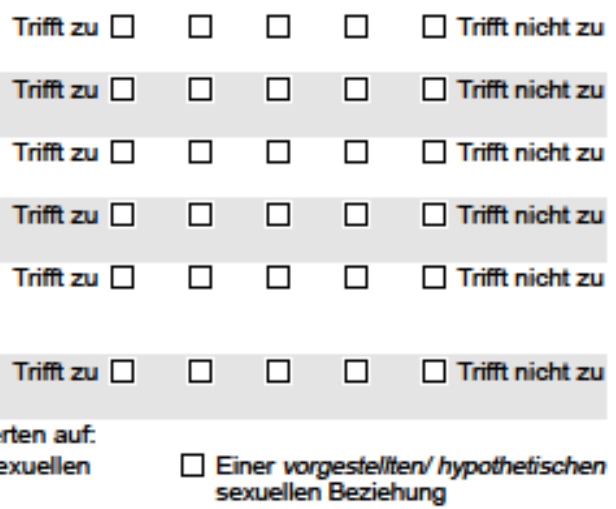

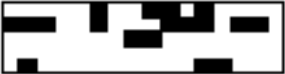


\begin{tabular}{l|c|c|} 
EvaSys & Sexuelle Gesundheit in der Altenpflege [Copy]
\end{tabular}

\section{Offenheit in der Familie}

7.1 Wie häufig wurde in meiner Familie über Sexualitāt gesprochen? Sehr häufig $\square \quad \square \quad \square \quad \square \quad \square$ Gar nicht

Wäufig

7.2 Wie offen wurde in meiner Familie über Sexualität gesprochen? $\quad$ Offen $\quad \square \quad \square \quad \square \quad \square \quad \square$ Gar nicht

7.3 Wie offen haben meine Eltern mit mir über Sexualität $\quad$ Sehr offen $\begin{array}{llll}\quad & \quad & \square & \square\end{array}$ Gar nicht gesprochen?

7.4 Wie offen haben meine Eltern mit mir über ihre eigene $\quad$ Sehr offen $\quad \square \quad \square \quad \square \quad \square \quad \square$ Gar nicht Sexualität gesprochen?

7.5 Für wie aufgeklärt halte ich mich persönlich?

$\underset{\text { aufgeklärt }}{\text { Sehr }} \quad \square \quad \square \quad \square \quad \square$ Gar nicht

7.6 Von wem bin ich hauptsächlich aufgeklärt worden? Mehrfachnennungen möglich.
$\square$ Fernsehen
$\square$ Freunde
$\square$ Internet
$\square$ Mutter
$\square$ Zeitschriften
$\square$ Geschwister
$\square$ Vater

Eltern aufgeklärt worden:

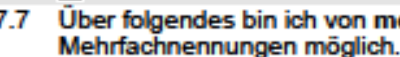
$\square$ Geburt
$\square$ Homosexualität
$\square$ Geschlechtskrankheiten
$\square$ Selbstbefriedigung
$\square$ Monatliche Blutung (Periode)
$\square$ Sexuelle Funktionsstörungen
$\square$ Schwangerschaft
$\square$ Verhütung (Pille, Kondome, Spirale...)

$\square$ Gar nicht

8. Meinungsbild

8.1 Gönnen Sie es alten Menschen, aktiv ihre Sexualität auszuleben?

Wieviel meinen Sie, wissen die Bewohner des Altenheim
im Durchschnitt über sexuell übertragbare Krankheiten?

$\square$ Ja

$\square$ Nein

$\square$ Weiß nicht

8.3 Wieviel meinen Sie, wissen Altenpfleger im Durchschnitt über sexuell übertragbare Krankheiten?

8.4 Wie viel wissen thre Schüler Ihrer Meinung nach über für den Berufsalltag wichtige sexuell übertragbare Krankheiten?

8.5 Wie wichtig denken Sie ist es Schüler über für den Berufsalltag wichtige sexuell übertragbare Krankheiten aufzuklären?

8.6 Wird von Seiten der Pflege darauf geachtet, dass es zu Vorsorgeuntersuchungen von Ärzten der Gynäkologie bzw. Urologie kommt?

8.7 Wären solche Arztbesuche lhrer Meinung nach sinnvoll?

8.8 Mit welchen der folgenden sexuell übertragbaren Erkrankungen hatten Sie in Ihrem Berufsalltag in der Altenpflege schon zu tun? Mehrfachnennungen möglich.
$\square$ Chlamydien
$\square$ Herpes genitales (HSV)
$\square$ Gonornhoe (Tripper)
$\square$ Human Immunodeficiency Virus (HIV)
$\square$ Pilzinfektionen, z.B. Scheidenpilze
$\square$ Treponema pallidum (Syphilis)
Gar nichts $\square$
$\square \quad \square$
$\square \quad \square$ Viel
Gar nichts

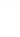
$\square$
$\square \quad \square$ Viel

$\square$ Hepatitis B und C

$\square$ Humanes Papilloma Virus (HPVFeigwarzen)

$\square$ Trifft nicht zu (keine Berufserfahrung in der Altenpflege) 


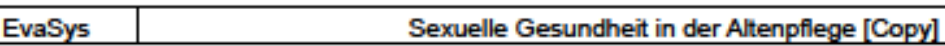

\section{Meinungsbild [Fortsetzung]}

8.9 Finden Sie die Bereitstellung sexueller Hilfsmittel von einer Einrichtung sinnvoll? Mehrfachnennungen möglich.
$\square$ Ja, Dildos
$\square \mathrm{Ja}$, Gleitgel
$\square$ Ja, Potenzmittel (z.B. Viagra)
$\square \mathrm{Ja}$, Vibratoren
$\square \mathrm{Ja}$, Penisringe
$\square$ Ja, Zeitschriften mit
$\square$ Nein
$\square \mathrm{Ja}$, Videos/ Filme mit pornografischen Inhalten
$\square$ Weiß nicht pornografischen Inhalten

$\square \mathrm{Ja}$, sonstige

8.10 Die folgenden Hilfsmittel sollten neben den hier genannten sexuellen Hilfsmittel in einer Einrichtung zur Verfügung gestellt werden:

8.11 Mehrfachnennungen möglich. Die Bereitstellung von sexuellen Hilfsmitteln

$\square$ Halte ich für sinnvoll, weil Bewohner $\square$ Halte ich für sinnvoll, weil es im selber keine beschaffen kōnnen Alter bei sexuellen Handlungen zu körperlichen

Funktionseinschrānkungen kommt

$\square$ Halte ich für sinnvoll, aber denke, dass es zu teuer ist

$\square$ Halte ich nicht für sinnvoll, da ältere Menschen nicht mehr sexuell aktiv sind

$\square$ Halte ich für sinnvoll, weil viele ältere Menschen noch sexuell aktiv sein wollen

$\square$ Halte ich nicht für sinnvoll, da ältere Menschen trotz Hilfsmittel

$\square$ Halte ich nicht für sinnvoll, da das Ausleben von Sexualität im $\square$ Weiß nicht alltere Menschen trotz Hilfsmitte
nicht sexuell aktiv sein können Alter abstoßend ist

\begin{tabular}{|c|c|c|c|c|}
\hline 8.12 & $\begin{array}{l}\text { Können Frauen auch nach den Wechseljahren } \\
\text { noch Orgasmen haben? }\end{array}$ & $\begin{array}{l}\square \text { Ja, alle Frauen } \\
\square \text { Nein, keine Frau }\end{array}$ & $\begin{array}{l}\square \text { Ja, viele Frauen } \\
\square \text { Weiß nicht }\end{array}$ & $\square \mathrm{Ja}$, wenige Frauen \\
\hline 8.13 & $\begin{array}{l}\text { Können Frauen aus biologischen Gründen } \\
\text { sexuelle Abstinenz besser ertragen als Männer? }\end{array}$ & $\square \mathrm{Ja}$ & $\square$ Nein & $\square$ Weiß nicht \\
\hline 8.14 & Ist Homosexualitāt eine Krankheit? & $\square \mathrm{Ja}$ & $\square$ Nein & $\square$ Weiß nicht \\
\hline 8.15 & $\begin{array}{l}\text { Ist der Mann aus biologischen Gründen sexuell } \\
\text { aktiver als die Frau? }\end{array}$ & $\square \mathrm{Ja}$ & $\square$ Nein & $\square$ Weiß nicht \\
\hline 8.16 & Ist hāufiges Masturbieren psychisch schädlich? & $\square$ Ja & $\square$ Nein & $\square$ Weiß nicht \\
\hline \multicolumn{5}{|c|}{ 9. Allgemeiner Teil } \\
\hline 9.1 & Alter & $\begin{array}{l}\square<25 \text { Jahre } \\
\square \text { 45-55 Jahre }\end{array}$ & $\begin{array}{l}\square \text { 25-35 Jahre } \\
\square \text { 55-60 Jahre }\end{array}$ & $\begin{array}{l}\square \text { 35-45 Jahre } \\
\square>60 \text { Jahre }\end{array}$ \\
\hline 9.2 & Geschlecht & $\square$ Männlich & $\square$ Weiblich & Sonstiges \\
\hline 9.3 & $\begin{array}{l}\text { Beziehungsstatus } \\
\square \text { Feste Partnerschaft } \\
\square \text { Verheiratet }\end{array}$ & $\begin{array}{l}\square \text { Offene Beziehung } \\
\square \text { Verwitwet }\end{array}$ & \multicolumn{2}{|l|}{$\begin{array}{l}\square \text { Single } \\
\square \text { Sonstiges }\end{array}$} \\
\hline 9.4 & $\begin{array}{l}\text { Sexuelle Orientierung } \\
\square \text { Asexuell } \\
\square \text { Homosexuell }\end{array}$ & $\begin{array}{l}\square \text { Bisexuell } \\
\square \text { Sonstiges }\end{array}$ & \multicolumn{2}{|c|}{$\square$ Heterosexuell } \\
\hline 9.5 & $\begin{array}{l}\text { Derzeit in der Altenpflege aktiv } \\
\square \text { Ja }\end{array}$ & $\square$ Nein & \multirow{2}{*}{$\square$ Nein } & \multirow[b]{3}{*}{$\square$ Privat } \\
\hline 9.6 & Studienabschluss & $\square \mathrm{Ja}$ & & \\
\hline 9.7 & Träger der Altenpflegeschule & $\begin{array}{l}\square \text { Kirche } \\
\square \text { Wohlfahrtsverband }\end{array}$ & $\begin{array}{l}\square \text { Öfentlich } \\
\square \text { Sonstiges }\end{array}$ & \\
\hline 9.8 & \multicolumn{4}{|c|}{$\begin{array}{l}\text { Auf welchem Weg sind Sie zu lhrer Qualifikation als Lehrender an einer Altenpflegeschule gelangt? } \\
\text { Bitte unter Angabe der Ausbildung/ Studium/ Fortbildung und des Abschlussjahres. }\end{array}$} \\
\hline
\end{tabular}




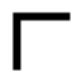

\begin{tabular}{l|c} 
EvaSys & Sexuelle Gesundheit in der Altenpflege [Copy]
\end{tabular}

\section{Allgemeiner Teil [Fortsetzung]}

9.9 Berufserfahrung im jetzigen Beruf

$\square<1$ Jahr

$\square$ 5-10 Jahre

$\square$ 1-3 Jahre

$\square$ 10-20 Jahre

$\square$ 3-5 Jahre

9.10 Jetziger Beruf mit Fachrichtung

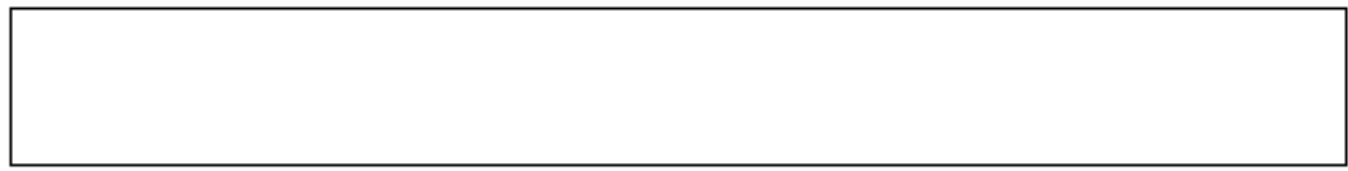

\section{Anmerkungen}

10.1 Wünsche und Anregungen sind an dieser Stelle wilkommen. 


\section{$7 \quad$ Literaturverzeichnis}

Ahrold TK, Meston CM (2010): Ethnic differences in sexual attitudes of U.S. college students: gender, acculturation, and religiosity factors. Arch Sex Behav 39, 190-202

Aström S, Bucht G, Eisemann M, Norberg A, Saveman B-I (2002): Incidence of violence towards staff caring for the elderly. Scand J Caring Sci 16, 66-72

Bauer M, McAuliffe L, Nay R, Chenco C (2013): Sexuality in Older Adults: Effect of an Education Intervention on Attitudes and Beliefs of Residential Aged Care Staff. Educ Gerontol 39, 82-91

Bode H, Heßling A: Jugendsexualität 2015. Die Perspektive der 14- bis 25-Jährigen.

Ergebnisse einer aktuellen Repräsentativen Wiederholungsbefragung. Bundeszentrale für gesundheitliche Aufklärung, Köln 2015

Bogner K, Landrock U (2015): Antworttendenzen in standardisierten Umfragen. Mannheim, GESIS Leibniz Institut für Sozialwissenschaften (SDM Survey Guidelines). DOI: 10.15465/gesis-sg_016

Bouman WP, Arcelus J, Benbow SM (2007): Nottingham study of sexuality and ageing (NoSSA II). Attitudes of care staff regarding sexuality and residents: A study in residential and nursing homes. Sex Relation Ther $\underline{22}, 45-61$

Brähler E, Böhm A: Einstellungen zur Sexualität und sexualmedizinische Kenntnisse von zukünftigen Ärzten/Ärztinnen. In: Brähler E, Meyer A (Hrsg.): Partnerschaft, Sexualität und Fruchtbarkeit. Springer, Berlin 1988, 277-291

Bronner G, Peretz C, Ehrenfeld M (2003): Sexual harassment of nurses and nursing students. J Adv Nurs. 42, 637-644

Bucher T, Hornung R, Buddeberg C (2003): Sexualität in der zweiten Lebenshälfte. Ergebnisse einer empirischen Untersuchung. Z Sexualforsch 16, 249-270

Buchner J, Maier V, Ullmann R, Garten T: Sexualität im Alter. Ergebnisse einer Pilotstudie der Helios Klinik Berching. In: Bach D, Böhmer F (Hrsg.): Intimität, Sexualität, Tabuisierung im Alter. Böhlau Verlag, Wien 2011, 69-95

Bundesministerium für Familie, Senioren, Frauen und Jugend (Hrsg.): Altenpflegeausbildung. Informationen zu Ausbildung und Beruf der Altenpflegerinnen und Altenpfleger. 5. Auflage; Bonifatius GmbH, Paderborn 2016

de Vries N: Erfahrungsbericht aus der Sexualassistenz. In: Bach D, Böhmer F (Hrsg.): Intimität, Sexualität, Tabuisierung im Alter. Böhlau Verlag, Wien 2011, 147-155

Glass JCJ, Mustian RD, Carter LR (1986): Knowledge and Attitudes of Healthcare Providers Toward Sexuality in the Institutionalized Elderly. Educ Gerontol 12, 465475 
Franzen A: Antwortskalen in standardisierten Befragungen. In: Baur N., Blasius J. (Hrsg.): Handbuch Methoden der empirischen Sozialforschung. Springer VS, Wiesbaden 2014 Gott CM (2001): Sexual activity and risk-taking in later life. Health Soc Care Community $\underline{9}$, $72-78$

Grond E: Sexualität im Alter. (K)ein Tabu in der Pflege. 1. Auflage; Brigitte Kunz Verlag, Hagen 2001

Grond E: Sexualität im Alter. Was Pflegekräfte wissen sollten und was sie tun können. 2. Auflage; Brigitte Kunz Verlag, Hagen 2011

Hallauer JF, Bienstein C, Lehr U, Rönsch H: SÄVIP - Studie zur ärztlichen Versorgung in Pflegeheimen. Vincentz Network, Hannover 2005

Herkner H, Müllner M: Erfolgreich wissenschaftlich arbeiten in der Klinik. Grundlagen, Interpretation und Umsetzung: Evidence Based Medicine. 3. Auflage; Springer, Wien 2011

Herold G: Innere Medizin: Eine vorlesungsorientierte Darstellung. Auflage 2016; Herold Verlag, Köln 2016

Hillman JL, Stricker G (1994): A Linkage of Knowledge and Attitudes Toward Elderly Sexuality: Not Necessarily a Uniform Relationship. Gerontologist 34, 256-260

Kleinevers S: Sexualität und Pflege. Bewusstmachung einer verdeckten Realität. 1. Auflage; Schlütersche, Hannover 2004

Krebs D (1991): Was ist sozial erwünscht? Der Grad sozialer Erwünschtheit von Einstellungsitems. Zentrum für Umfragen, Methoden und Analysen - ZUMAArbeitsbericht $\underline{18}$

Krosnick JA, Narayan S, Smith WR (1996): Satisficing in Surveys: Initial evidence. Advances in Survey Research $\underline{70}, 29-44$

Krosnick JA, Presser S: Question and Questionnaire Design. In: Wright JD, Marsden PV (Hrsg.): Handbook of Survey Research. 2. Auflage; Elsevier, San Diego 2009

Lehmann A: Psychosoziale Einflussfaktoren auf das weibliche Sexualerleben. Phil. Diss. Berlin 2007

Leitz S, Signerski-Krieger J (2018): Sexualität der Vielfalt? Eine empirische Untersuchung niedersächsischer Biologiebücher. Z Sexualforsch $\underline{31}$, 356-377

Lewis S, Bor R (1994): Nurses' knowledge of and attitudes towards sexuality and the relationship of these with nursing practice. J Adv Nurs 20, 251-259

Matolycz E: Pflege von alten Menschen. Springer, Wien 2011

Meudt D: Sexualität in der Pflege alter Menschen. Ein Ausbildungsmodul für die Altenpflege. Kuratorium Deutsche Altershilfe, Köln 2006 
Michelchen G: Intimität in der Altenpflege. Tabus in Pflegeeinrichtungen überwinden. 1. Auflage; CW Haarfeld, München 2008

Niedersächsischer Landtag (Hrsg.): Schule muss der Vielfalt sexueller und geschlechtlicher Identitäten gerecht werden -Persönlichkeitsentwicklung der Kinder und Jugendlichen fördern -Diskriminierung vorbeugen. Niedersächsischer Landtag-17. WahlperiodeDrucksache 17/2585. o. Verl., Hannover 2014

Niedersächsisches Kultusministerium (Hrsg.): Rahmenrichtlinien für den berufsbezogenen Lernbereich in der Berufsfachschule-Altenpflege. o. Verl., Hannover 2003

Porst R: Fragebogen. Ein Arbeitsbuch. 4. Auflage; VS Verlag für Sozialwissenschaften, Wiesbaden 2014

Schmidt R-B: Schule als Ort sexueller Sozialisation. In: Hagedorn J (Hrsg.): Jugend, Schule und Identität. Selbstwerdung und Identitätskonstruktion im Kontext Schule. Springer VS, Wiesbaden 2014, 249-264

Sielert U: Expertise zum Thema „Sexualerziehung in Grundschulen“. Institut für Sozialpädagogik der Universität Kiel, Kiel 2011

Sielert U: Einführung in die Sexualpädagogik. 2. Auflage; Beltz Verlag, Weinheim 2015 Sieren K: Sexuelle Bedürfnisse von Altenheimbewohnern. Empirische Studie zu einem Tabuthema. Diplomica Verlag GmbH, Hamburg 2012

Snell WE Jr, Fisher TD, Walters AS (1993): The Multidimensional Sexuality Questionnaire: An Objective Self-Report Measure of Psychological Tendencies Associated with Human Sexuality. Ann Sex Res $\underline{6}, 27-55$

Statistisches Bundesamt: Die Generation 65+ in Deutschland. Destatis, Wiesbaden 2015 Statistisches Bundesamt: Pflegestatistik 2015. Pflege im Rahmen der Pflegeversicherung.

Deutschlandergebnisse. Destatis, Wiesbaden 2017

Turner D, Driemeyer W, Nieder TO, Scherbaum N, Briken P (2014): „Wie viel Sex braucht das Studium der Medizin?"“ - Eine Erhebung des Wissens und Interesses Medizinstudierender zum Thema Sexualmedizin. Psychother Psych Med 64, 452-457 van der Vight-Klußmann R: (Kein) Sex im Altenheim? Körperlichkeit und Sexualität in der Altenhilfe. Schlütersche, Hannover 2014

von Simson R, Kulasegaram R (2012): Sexual health and the older adult. Stud BMJ 20, e688 Wendland S (2013): Sexualität- (K)ein Thema in der Altenpflege? Pro Familia Magazin 4 , 14-15

Zettl S: Krankheit, Sexualität und Pflege. Hilfestellungen für den Umgang mit einem Tabu. 1. Auflage; W. Kohlhammer, Stuttgart 2000 
Zettl-Wiedner K: Hat Sexualität im Alter in einer Langzeitpflegesituation überhaupt Platz? In: Bach D, Böhmer F (Hrsg.): Intimität, Sexualität, Tabuisierung im Alter. Böhlau Verlag, Wien 2011, 123-130

\subsection{Internetquellen}

Bundesministerium für Gesundheit (2014): Glossar, Begriffe A-Z. Heim. https://www.bundesgesundheitsministerium.de/service/begriffe-von-az/h/heim.html; abgerufen am 10.01.2020

Bundeszentrale für politische Bildung (2014): 1994: Homosexualität nicht mehr strafbar. https://www.bpb.de/politik/hintergrund-aktuell/180263/1994-homosexualitaetnicht-mehr-strafbar; abgerufen am 13.04.2020

Heimverzeichnis gGmbH. Altenheime, Pflegeeinrichtungen und Seniorenresidenzen mit Lebensqualität.

https://www.heimverzeichnis.de/index.php?id=12\&f_su_ort $=\mathrm{g} \% \mathrm{C} 3 \% \mathrm{~B} 6$ ttingen\&f_s u_ent $=10 \& f$ _sop_traeg $=0 \& a r t$ der_einr $=0 \& f$ _su_submit $3 . x=0 \& f$ su_submit $3 . y=0 \& f$ _sp_161=0\&f_sp_ang=; abgerufen am 10.06.2017

Multidimensional Sexual Self-Concept Questionnaire. https://openpsychometrics.org/tests/MSSCQ.php; abgerufen am 12.10.2017

Pflegekammer Niedersachsen KdöR.

http://www.pflegekammer-niedersachsen.de/index.php/de/ausbildungsstaetten-inniedersachsen/schulen-fuer-altenpflege.html; abgerufen am 06.06.2017

WHO-Regionalbüro für Europa (2011): Sexuelle und reproduktive Gesundheit. http://www.euro.who.int/de/health-topics/Life-stages/sexual-and-reproductivehealth/news/news/2011/06/sexual-health-throughout-life/definition; abgerufen am 06.10.2017

WHO-Regionalbüro für Europa und BZgA (2011): Standards für die Sexualaufklärung in Europa. Rahmenkonzept für politische Entscheidungsträger, Bildungseinrichtungen, Gesundheitsbehörden, Expertinnen und Experten https://www.bzgawhocc.de/fileadmin/user_upload/WHO_BZgA_Standards_deutsc h.pdf; abgerufen am 02.12.2019 


\section{Danksagung}

Herrn Prof. J. Wiltfang möchte ich als Leiter der Klinik für Psychiatrie und Psychotherapie der Universität Göttingen besonders für die Möglichkeit einer Promotion an seinem Institut danken. Herrn Prof. J. Wiltfang als meinem Doktorvater und Herrn Prof. W. Himmel möchte ich für die stets konstruktiven Kritiken und Vorschläge während unserer gemeinsamen Treffen danken.

Meiner Betreuerin Frau Dr. S. Meyer-Rötz möchte ich für die Hilfe, Unterstützung und den organisatorischen Beistand besonders in den Anfängen der Studie danken. Meinem Betreuer Herrn Dr. J. Signerski-Krieger möchte ich für die unzähligen, immer unterhaltsamen, produktiven Treffen über die gesamte Zeit und insbesondere in der Endphase der Promotion danken! Es war mir eine große Freude mit ihm zusammen zu arbeiten. Ebenso bedanke ich mich für die Freiheiten, die mir während der Vorbereitung der Studie überlassen worden sind. Vielen Dank für die seelische Unterstützung und der Ermutigung, wenn (wie immer) nicht alles nach Plan lief.

Ich danke allen Mitarbeiterinnen des Instituts für Studium und Lehre, die mir während des Einscannens meiner Fragebögen beigestanden und geholfen haben.

Meiner guten Freundin Emilie Sievert danke ich für die Möglichkeit, mir den Zugang zu vielen interessanten und spannenden Quellen zu ermöglichen.

Ein riesengroßes Dankeschön gilt allen teilnehmenden Altenpflegenden, Auszubildenden und Lehrenden der Altenpflegeheime und -schulen, denn ohne ihren Einsatz und ihre Geduld wäre diese Dissertation nicht möglich gewesen. 